\title{
Molecular Modelling of Ionic Liquids: Force-Field Validation and \\ Thermodynamic Perspective from Large-Scale Fast-Growth Solvation Free \\ Energy Calculations
}

Zhaoxi Sun ${ }^{1 *}$, Mao Wang ${ }^{2}$, Qiaole $\mathrm{He}^{3}$, Zhirong Liu ${ }^{1}$

\begin{abstract}
${ }^{I}$ Beijing National Laboratory for Molecular Sciences, Institute of Theoretical and Computational Chemistry, College of Chemistry and Molecular Engineering, Peking University, Beijing 100871, China

${ }^{2}$ NCS Testing Technology Co., Ltd., No. 13, Gaoliangqiao Xiejie, Beijing 100081, China

${ }^{3}$ AI Department of Enzymaster (Ningbo) Bio-Engineering Co., Ltd., North Century Avenue 333, 315100 Ningbo,
\end{abstract}

China

*To whom correspondence should be addressed: z.sun@pku.edu.cn

\begin{abstract}
Molecular simulations are becoming a common tool for the investigation of dynamic and thermodynamic properties of novel solvents such as ionic liquids and the more recent deep eutectic solvents. As the electrostatics derived from ab initio calculations often fail to reproduce the experimental behaviors of these functionalized solvents, a common treatment is scaling the atomic charges to improve the accord between experimental and computational results for some selected properties, e.g., the density of the liquids. Although there are many computational benchmarks on structural properties of bulk ionic liquids, the choice of the best scaling parameter remains an open question. As these liquids are designed to solvate solutes, whether the solvation thermodynamics could be correctly described is of utmost importance in practical situations. Therefore, in the current work, we provide a thermodynamic perspective of this charge scaling issue directly from solute-solvent interactions. We present a comprehensive large-scale calculation of solvation free energies via nonequilibrium fast-switching simulations for a spectrum of molecules in ionic liquids, the atomic charges of which derived from ab initio calculations are scaled to find the best scaling factor that maximizes the prediction-experiment correlation. Further, the density-derived choice of the scaling parameter, which is used as the estimate from bulk properties, is computed and compared with the
\end{abstract}


solvation-free-energy-derived one. It is observed that when the scaling factor is decreased from 1.0 to 0.5 , the mass density exhibits a monotonically decreasing behavior, which is caused by weaker inter-molecular interactions produced by the scaled atomic charges. However, the solvation free energies of external agents do not show consistent monotonic behaviors like the bulk property, the underlying physics of which are elucidated to be the competing electrostatic and vdW responses to the scaling-parameter variation. More intriguingly, although the recommended value for charge scaling from bulk properties falls in the neighborhood of $0.6 \sim 0.7$, the solvation free energies calculated at this value are not always in good agreement from the experimental reference. By modestly increasing the scaling parameter (e.g., by 0.1$)$ to avoid over-scaling of atomic charges, the solute-solvent interaction free energy approaches the reference value and the quality of calculated solvation thermodynamics approaches the hydration case. According to this phenomenon, we propose a feasible way to obtain the best scaling parameter that produces balanced solute-solvent and solvent-solvent interactions, i.e., first scanning the density-scaling-factor profile and then adding $\sim 0.1$ to that solution. We further calculate the partition coefficient or the transfer free energy of solutes from water to ionic liquids to provide another thermodynamic perspective of the charge scaling benchmark. Another central result of the current work is about the widely used force fields to describe bonded and vdW terms for ionic liquids derivatives (i.e., the GAFF derivatives). These pre-fitted transferable parameters are evaluated and refitted in a system-specific manner to provide a detailed assessment of the reliability and accuracy of these commonly used parameters. Component-specific refitting procedures unveil that the bond-stretching term is the most problematic part of the GAFF derivatives in modelling ionic liquids and the angle-bending term in some cases is also not accurate enough. Astonishingly, the torsional potential defined in these pre-fitted force fields performs extremely well.

Keywords: Ionic Liquids, Charge Scaling, Force Field, Fast Growth, Solvation Free Energy 


\section{Introduction}

The development of green solvents for chemical and biomedical applications is of increasing emergence. Ionic liquids and their more recent analogues deep eutectic solvents are promising replacements for conventional organic volatile, toxic and/or flammable solvents due to their tunability, lack of flammability, etc. ${ }^{1-9}$ More importantly, these functionalized solvents exhibit satisfactory solvation power for organic compounds and metallic species. ${ }^{10-13}$ For instance, these novel solvents are applied as extractants in the separation of aromatic hydrocarbon from naphtha and in the extraction of metals. ${ }^{14-16}$ Many polymeric species that are distinct from water also show satisfactorily high solubilities in these functionalized solvents. ${ }^{17}$ These novel solvents also find their positions in analytical chemistry through separation applications in chromatography as stationary phases, mobile phase additives or flow modifiers. ${ }^{18-21}$ When investigating the solvation process of some selected molecules in ionic liquids derivatives which is the most common and designed usage of these green solvents, the thermodynamic quantity with the highest relevance is, obviously, the solvation free energy.

Molecular dynamics simulations are becoming a common tool for the investigation of the dynamic and thermodynamic properties of complex biological and chemical systems. ${ }^{22-31}$ In molecular simulations of these green solvents, classical fixed-charge force fields are often incorporated to describe the intra- and inter-molecular interactions. ${ }^{32-38}$ The functional forms of classical force fields are simple enough, which enables large-scale explorations of long-time dynamics in complex solution systems. Complicated interaction networks are formed by complex ingredients of ionic liquids. Accurate modelling of ionic liquids requires appropriate and balanced descriptions of hydrogen bonding, long-range electrostatics, dispersion interactions, etc. In the formulism of classical force fields, these interactions are mainly defined in bonded, electrostatic and vdW terms. ${ }^{39-44}$ The bonded interactions determine the intra-molecular conformational preference and the non-bonded interactions including electrostatic and vdW interactions determine the strength and pattern of inter-molecular coordination.

The most widely accepted treatment for electrostatics is deriving atomic charges based on some quantum mechanical calculations. However, the electrostatics derived from ab initio calculations often fail to reproduce the experimental behaviors of these novel solvents. Although the careful parametrization of forcefield parameters for ionic liquids targeting some high-level $a b$ initio or experimental data could be performed, the most common, computationally efficient albeit less theoretically rigorous solution to this problem is scaling the atomic charges to improve the accord between experimental and computational results for some selected properties (e.g., the density of the system), the validity of which is often attributed 
to charge transfer and polarization. ${ }^{32,34,36-38,45}$ Despite the massive emphasize of bulk properties of these novel solvents in molecular simulations, little attention is paid to the most important thermodynamic observable that must be accurately reproduced in practical applications of ionic liquids derivatives, the solvation behavior of external agents in these functionalized solvents. Solvation free energy is often considered as a critical property in the parameterization and evaluation of modern force fields. ${ }^{46-50}$ Accurate calculation of the solvation properties of molecules of interest in different solvents is of utmost importance in the accurate modelling of complex biochemical processes. Although insights about charge scaling from this crucial thermodynamic quantity (i.e., solvation free energy) would be extremely useful, this critical observable as a criterion for the charge scaling issue is commonly overlooked. Considering the importance of such extensive benchmark calculations for this thermodynamic observable, in this work, we employ advanced sampling protocols to obtain the solvation free energies of a spectrum of small molecules in several ionic liquids. This thermodynamic perspective from solvation free energies is highly relevant to the balanced description of solute-solvent and solvent-solvent interactions. Thus, it serves as a criterion with higher priority than bulk properties in the modelling of ionic liquids. To avoid biasing the conclusion due to intrinsic limitations of the benchmark set, we select three ionic liquids and a large set of solute molecules with diverse structural features. Specifically, the cationic specie 1-ethyl-3-methylimidazolium (EMIM) is combined with three anionic species including dicyanamide (DCA), bis(trifluoromethylsulfonyl)imide $\left(\mathrm{Tf}_{2} \mathrm{~N}, \mathrm{NTf}_{2}\right.$, or NTf2), and trifluoromethylsulfonate (TfO or OTf) to form three room-temperature ionic liquids, all of which are typical ionic solvents used in industrial and laboratorial applications. These combined ionic mixtures are represented by the ion-pair abbreviations as [EMIM][DCA], [EMIM][NTf2] and [EMIM][OTf].

Aside from the charge scaling issue, whether the other parameters in commonly used classical force fields are suitable for ionic liquids derivatives is also unclear. Specifically, the massive application of the bonded and vdW parameters from the general AMBER force field (GAFF) parameterized for drug-like molecules is unjustified. Although the vdW parameters are often considered to be less system-dependent and thus are often used in a brute-force way, the dihedral or torsional terms in the bonded parameters that determine the intra-molecular conformational preference could show obvious system dependence. To solidate the whole modelling protocol for ionic liquids derivatives, it is inevitable to evaluate the reliability of these factors. Therefore, another focus of the current work is the assessment of the existing parameters from GAFF derivatives. Another evaluation step taken further is refitting the GAFF derivatives in a systemspecific manner to approach the accuracy limit under this functional form, which enables the identification 
of problematic force-field terms in the GAFF framework and provides hints about directions for further development. Overall, the current extensive computational investigation of ionic liquids and their interactions with external agents provides a useful and practical thermodynamic perspective for accurate modelling and efficient simulation of ionic liquids derivatives.

\section{Computational Setup}

\subsection{System Construction.}

As mentioned in the previous section, the current work aims at providing general guidelines for the selection of the scaling parameter for atomic charges in the simulations of ionic liquids derivatives. Thus, three different but typical ionic liquids used in industrial and laboratorial applications are considered. To make the observation of the solvation behavior broadly applicable and avoid biased conclusions due to limited solute selection, a spectrum of small drug-like molecules with diverse structural features are simulated. The solvation free energies of these molecules in water are also calculated to validate the model construction and simulation protocol used in the current work. The chemical structures of ions forming the considered ionic liquids are presented in Fig. 1, while the names of all solutes under consideration are summarized in Table S1. These experimental values of thermodynamic parameters are obtained from references. ${ }^{51-59}$ Note that for each ionic solvent, the solutes under consideration are a subset of the whole Table S1 list, and the details of these solute-solvent pairs would be presented in Table S2-S11 along with the computed results.

The 3D chemical structures of all molecules except water used in this study are obtained from the PubChem database. We then consider the parameterization of these species. For charge generation, we employ the restrained electrostatic potential (RESP) ${ }^{60}$ fitting with the traditional gas-phase scanning procedure. Namely, we optimize each molecule at the B3LYP/6-31G* level in vacuo, after which the electrostatic potential (ESP) around the molecule is scanned with the Merz-Kollman scheme at the HF/6$31 \mathrm{G}^{*}$ level. Then, restrained fitting with a two-step hyperbolic regularization term is then initiated to get the atom-centered charge set. The other terms (bonded and vdW interactions) are grabbed from the second generation of GAFF (GAFF version 2.11, GAFF2). ${ }^{61}$ Note that the model construction is also repeated with the first generation of GAFF (GAFF 1.81), but according to the results of the force-field evaluation presented in the results section, these two force fields show similar behaviors and thus only GAFF2 is used in simulations. When dealing with systems including water molecules (i.e., solvation in water), the TIP3P model $^{62,63}$ is employed. 
The PACKMOL package ${ }^{64}$ is used to construct the simulated solution box. The solvation of the considered molecules is expected to happen in a box of solvent molecules (i.e., ionic liquids or water) that produce the bulk properties. If the simulation box is not large enough, finite-size effects would be introduced and the structural arrangement of the solvent ${ }^{33}$ and thus solute-solvent interactions would be altered. Thus, the size of the simulation box and the number of solvent molecules presented should be sufficiently large to ensure that the bulk properties of the solvent are produced. For ionic liquids, this often indicates that the number of ion pairs should be sufficiently large to converge various properties. For each ion pair, the same amounts of cations and anions (i.e., ion pair) forming the ionic liquids are inserted into the cubic cell with a side length of $\sim 37 \AA$, and the number of the distributed solvent molecules (cations and anions forming ionic liquids) is calculated to make the number of atoms in the cubic box close to 5100 atoms. According to numerical tests, the simulation box of this size is sufficiently large to eliminate any finite-size effects. The cubic cell is replicated in whole space with periodic boundary conditions.

As the initial configuration obtained in this way is often suboptimal and far from equilibrium, we perform minimization and $20 \mathrm{~ns}$ NPT equilibration at the physiological condition (i.e., $298 \mathrm{~K}$ ) to get an equilibrated box. After that, we perform $30 \mathrm{~ns}$ production run to estimate the mass density of the ionic liquids. Note that the simulations are performed separately under different scaling parameters for the ionic liquids. As good experiment-simulation agreements are often observed when the scaling parameter lies in the range of $0.6-0.8,{ }^{36}$ in our benchmark calculations the scaling parameters employed include $0.6,0.7$, and 0.8. Note that as this charge scaling treatment is not really rigorous and leads to worse reproduction of the $a b$ initio ESP (i.e., the reference in RESP fitting), in the scaling parameter scanning we do not use too dense spacing but select the increment of 0.1 . We also include a further smaller scaling factor 0.5 and the $a b$ initio derived unscaled 1.0 parameter set in unbiased simulations for density estimation to cover the possible range that the best experiment-simulation agreement could be achieved.

\subsection{Nonequilibrium Alchemical Transformation.}

It is common to estimate the solvation free energy of drug-like molecules with alchemical free energy calculations, which construct an artificial transformation pathway along the so-called alchemical order parameter. ${ }^{50,65-67}$ The end states are defined as fully coupled and decoupled solute-solvent systems, and the variation of free energies along the alchemical pathway is calculated to estimate the final solvation free energy. The nonequilibrium transformation technique, which enables the feasible calculation of the free energy difference between end states, is employed in the current work. Only equilibrium simulations at 
physically meaningful end states are conducted to grab initial conditions, while the rest of sampling is performed in nonequilibrium trajectories. The theoretical perspectives of the nonequilibrium free energy techniques can be found in many references. ${ }^{68-76}$ Here, we only detail the sampling protocol used in our nonequilibrium alchemical transformation.

The alchemical order parameter $\lambda$ is used to define the percentage of coupling between the solute and solvent (water). A value of zero suggests the fully decoupled state, while a value of one provides the physical solvated state. To properly treat the perturbation and structural rearrangement during the solvation process, we use long unidirectional pulling initiated from the decoupled state in the alchemical free energy calculation. The initial structure of solute is the optimized structure used in charge generation (i.e., gas-phase optimized one at the B3LYP/6-31G* level), and 5000 steps gradient decent and 2 ns equilibration are conducted to obtain an equilibrated condition, from which $100 \mathrm{~ns}$ unbiased sampling at the physiological condition with the sampling interval of $1 \mathrm{~ns}$ are performed to grab 100 initial conditions for nonequilibrium pulling. Note that this sampling interval is sufficiently long to effectively eliminate possible correlations in the simulated system, and the large number of independent initial configurations enables a good coverage of the relevant space. The $\lambda$-switching procedure is composed of two successive sub-steps. The solute-solvent $\mathrm{vdW}$ interaction is gradually turned on with the soft-core realization in the first step, after which the electrostatic part is switched on. As the transformation of this dispersive-repulsive interaction is often more challenging than the electrostatic part, the pulling time of the vdW change is set to $400 \mathrm{ps}$, while that of the electrostatics is $200 \mathrm{ps}$. The $\lambda$ variation is performed every $2 \mathrm{fs}$. As a result, the magnitudes of each $\lambda$ perturbation are 5e-6 and 1e-5 for the vdW and electrostatic transformations, respectively. The nonequilibrium transformation for each solvation free energy calculation thus lasts $600 \mathrm{ps}$, and the total 100 realizations lead to the pulling time of $60 \mathrm{~ns}$. By adding the equilibrium sampling length for initial configurations, the total sampling time of each solvation free energy calculation in equilibrium and nonequilibrium ensembles is $160 \mathrm{~ns}$. This sampling time in the current $\lambda$-switching protocol is much longer than the normally used one for solvation free energy calculations, thus often suggesting a satisfactory convergence behavior of the nonequilibrium free energy calculation. In the case that the convergence behavior of the nonequilibrium alchemical transformation is not very good, the details of which would be discussed in the results part later, we double the pulling time (i.e., $800 \mathrm{ps}$ vdW and 400 ps electrostatic transformations with a perturbation step of 2.5e-6 and 5e-6, respectively). The GROMACS 2019.6 implementation ${ }^{77}$ is used for the alchemical free energy calculation. The velocity rescaling algorithm ${ }^{78}$ is employed for temperature regulation at $298 \mathrm{~K}$ and the Parrinello-Rahman Lagrangian ${ }^{79,80}$ with isotropic 
scaling is used for pressure regulation. Long-range electrostatics are treated with the smooth Particle-mesh Ewald (PME) $)^{81}$ method. Note that the fast-switching solvation free energy calculations are also performed under different charge scaling parameters for ionic liquids to find the best option for this treatment. The scaling parameters tested would be discussed in the results section presented later.

\section{Results and Discussions.}

\subsection{Charge Generation.}

Electrostatics are considered to be the most flexible part of classical force fields. Therefore, this highly system-dependent component is often fitted in a molecule-specific manner. Despite the massive use of the RESP scheme in ionic liquids derivatives, whether the naïve application of the traditional fitting procedure is appropriate remains unclear. In the generation of RESP charges, the structure is optimized to reach a highly populated low-energy configuration, which is then used for ESP scan to obtain the fitting target. It should be noted that the reference value used in the regularized charge-fitting is the Coulombic molecular ESP, the accurate reproduction of which ensures the accuracy of inter-molecular electrostatic interactions. Therefore, it is straightforward to compare the ESP produced by the fitted charges with the ab initio reference in the current evaluation of the atomic charges. To this aim, we calculate the ESP relative root-mean-squared error (RRMSE) of the RESP charges with respect to the scanned HF/6-31G* reference, which is shown in the inset of Fig. 2a. For all ions forming the ionic liquids under consideration, the ESP RRMSEs are satisfactorily small, which suggests a good level of reproduction of the inter-molecular electrostatics at the target level. However, we should note that by scaling the atomic charges, the molecular ESP produced by atomic charges would deviate from the current fully charged results, which would degrade this ESP reproduction to some extent. The dipole moments produced by the RESP charges are compared with the $a b$ initio results also in Fig. 2a, where a satisfactory agreement is again observed, although this observable is not included in the objective function in the restrained fitting. Therefore, for fully charged ion pairs, the inter-molecular electrostatic interactions could be reproduced very well with ab initio accuracy. However, as the fixed-charge treatment could be inappropriate for highly charged species due to the neglection of charge transfer and polarization induced by environmental coupling, charge scaling is often applied in simulations employing fixed-charge classical force fields.

Accurate modelling of the solvation behavior also requires accurate electrostatics, but this time the interaction is formed between solute and solvent molecules. We then check the dipole moments produced by the RESP charges and the ab initio results for all solutes under consideration in Fig. 2b, where still 
satisfactory agreements are observed. A wide range of chemical features are covered by these selected solute molecules. For instance, the dipole moments of solutes cover the range of $0 \sim 4.5$ Debye, which suggests the inclusion of both highly polar and non-polar species in the dataset. These selected molecules could be considered as typical solutes for ionic liquids derivatives. As the RESP scheme produces satisfactory reproductions of both the molecular ESP and dipole moments for both ionic solvents and solutes, we conclude that the application of the RESP charge scheme in ionic-liquids simulations is generally not problematic. However, it should be noted that when charge scaling is applied to the ionic solvent, the atomic-charge-produced electrostatics are expected to deviate from the ab initio results, which is true for both solvent-solvent and solute-solvent interactions.

\subsection{Assessment of the GAFF Derivatives.}

After finishing the assessment of the most flexible part of force fields, we then turn to the less systemdependent parts, the parameters of which are often extracted from the pre-fitted GAFF derivatives in the modelling of ionic liquids derivatives. Although the GAFF parameters are commonly employed in the simulations of ionic liquids derivatives, its quality or closeness to high-level ab initio descriptions is seldom evaluated. As these general-purpose transferable parameters are pre-fitted from a large set of drug-like molecules, which could differ from species forming ionic liquids derivatives, whether the GAFF derivatives could produce satisfactory descriptions of these novel solvents remain unknown. Thus, the second part of our modelling of ionic liquids focuses on the justification of the use of these pre-fitted parameters and figures out whether further developments are in urgent need. As atomic charges are fitted in a systemspecific manner and GAFF derivatives are used to describe bonded and vdW interactions, the force-field parameters under assessment only include bonded and vdW terms. As vdW interactions often exhibit less system-dependent behaviors and play a relatively minor role in molecular recognition, these parameters are similar in different force fields. As a result, the main difference between different classical force fields lies in the bonded terms, which include potentials describing bond stretching, angle bending, and dihedral flipping. Among these stiff degrees of freedom, the dihedral/torsional terms are relatively soft and play a key role in determining the intra-molecular conformational preference of individual molecules involved in intermolecular arrangement. As a result, different torsional parameters could lead to significant differences in inter-molecular coordinations. ${ }^{82}$

A direct way to evaluate the goodness of a parameter set is calculating its deviations from some highquality reference data with a certain degree of reliability. For instance, we can select an ab initio level and 
calculate various properties of molecules under consideration as the reference values. Then, we repeat the same calculation using the selected force field and estimate the deviations of these results from the ab initio reference. However, such comparison only provides the absolute deviation of the selected parameter set from the reference data, which is influenced by both the functional form of the force field and the quality of the pre-fitted parameters. The former is considered as the intrinsic limitation introduced by the functional form used in the force field and cannot be overcome unless more complicated terms are added. However, this betterment often comes with a higher computational burden that is undesired for molecular simulation, especially for those requiring long-time-scale sampling. By contrast, the latter could be more straightforwardly improved by adjusting existing parameters, which approaches the accuracy limit of the employed functional form and does not increase the computational cost in sampling. A good force-field evaluation should provide information about both the 'badness' of the existing parameters used in the force field and its distance from the best solution under the existing functional form. Therefore, in the current assessment of the GAFF derivatives two goals are pursued. Here, we use GAFF2 as an example in presentation. The first goal is thus the estimation of the deviations from the reference level under the GAFF2 parameter set, and the second one is whether significant improvements could be gained by refitting the GAFF2 parameters to provide higher-accuracy descriptions. Below, we provide details about the force-field refitting and evaluation for all ionic liquids under investigation.

The generalized force-matching (FM) scheme $\mathrm{s}^{83,84}$ is employed in the force-field refitting. The other nonbonded terms (i.e., atomic charges and vdW terms) are kept unchanged during the course of the refitting. Although the 1-4 non-bonded interactions that are calculated between atoms separated by three consecutive bonds are often considered as bonded terms that correct the errors of the dihedral/torsional terms, as the other non-bonded parameters are kept fixed, the 1-4 non-bonded scaling constants are also excluded from the adjustable parameter set. As a result, only bonded parameters including bond stretching, angle bending, and dihedral flipping terms are optimized.

The objective function used in the current refitting includes the system energy, every component of the atomic forces, and a regularization term restraining the parameter space to explore and avoiding overfitting. The parameterization procedure is generally the same as our previous host-guest work. ${ }^{82}$ Specifically, the reference value of the harmonic L2 restraint imposed on all bonded terms is GAFF2. The relative weights of the energy and force terms are the same, while that of the regularization term is much smaller ( 0.1 of the other two terms). The prior widths remain their recommended values in the implementation. As tested in our previous works, this strength of regularization often triggers an error increase of $\sim 10 \%$ compared with the 
unregularized parameter adjustment, but the obtained parameters are effectively restrained in the reasonable range of physical properties. ${ }^{82}$ The parameter space is further regularized according to the atom-type symmetry defined in the initial guess of GAFF2. Note that the periodicity of dihedral terms is also fixed in the refitting.

Then we detail the procedure of the generation of reference data. The configurations used in $a b$ initio calculations are generated with classical force fields. For each molecule (ion), we accumulate 10000 configurations with a sampling interval of $10 \mathrm{ps}$, which leads to a sampling time of $100 \mathrm{~ns}$ for each ion. Instead of using the physiological condition, in this configurational sampling we employ a high temperature of $600 \mathrm{~K}$ in order to enable unconstrained exploration of the configurational space. The obtained 10000 configurations are then used for $a b$ initio calculations. The highly efficient B97-3c composite method $^{85}$ performs quite well in reproducing the high-level energetics for ionic liquids. ${ }^{33}$ Therefore, in the current force-field refitting and evaluation, this method is selected as the reference level and $\mathrm{ORCA}^{86,87}$ is used to perform these calculations. The optimized parameter set is named as FM-B97-3c in the following discussion.

After obtaining the optimized parameter set, we then perform some sampling to grab some configurations for quality assessment. In this procedure, we perform $10 \mathrm{~ns}$ gas-phase sampling with a sampling interval of 10 ps at $300 \mathrm{~K}$, which gives 1000 independent configurations. The energetics are calculated with the pre-fitted GAFF2 and the refitting FM-B97-3c parameter set and their deviations from the $a b$ initio reference are presented in Fig. S1, where we can see that the refitted parameter set produces results much closer to the reference level than the initial guess GAFF2. The root-mean-squared error (RMSE) of the energetics produced by the two parameter sets are provided in Fig. 3a, where we know that the GAFF2 error is quite large for all ions under consideration, while significant improvements are attained by adjusting all bonded terms in a regularized way to fit the ab initio data. This observation suggests that the refitting of the pre-fitted GAFF derivatives seems necessary for the modeling of ionic liquids derivatives. Although this information is very useful for accurate modelling of ionic liquids, it is still unclear that which term in force fields is more problematic, i.e., requiring adjustment. We thus look into the details of the adjusted parameter set and see which part is changed significantly upon refitting. Interestingly, this fitting-details check suggests the bond stretching interaction to be the most questionable part in GAFF derivatives for describing ionic liquids, and in some cases the angle term is also changed significantly. However, intriguingly, the variations of the rest of bonded terms (i.e., dihedral/torsional arrangement) are quite small. Thus, it is reasonable to wonder whether the bond stretching and sometimes angle bending terms are contributing almost all improvements in refitting and the dihedral interactions are not really problematic. To validate this hypothesis, we repeat the GAFF2 refitting with two other fitting procedures. In the first one, the refitting proceeds with only angle and dihedral 
terms included in the objective function and the bond stretching term is kept fixed. This scheme enables us to separate the energetic improvements due to bond stretching terms. In the second scheme, both bond stretching and angle bending terms are fixed and only the torsional term is under adjustment, which enables the direct evaluation of the quality of torsional parameters of GAFF2. As the focus here is the accuracy improvements after refitting with the all-terms-flexible, bond-stretching-fixed and dihedral-only procedures, we present the improvement of RMSE of the system energy, which is defined as the difference between the RMSE of GAFF2 and that of FM-B97-3c. As shown in Fig. 3b, although significant improvements are observed in refitting when all bonded terms are adjustable, the betterment is smaller under the bond-stretching-fixed procedure. The situation is extremely severe for the DCA anion, where it is clear that the bond stretching term is contributing almost all RMSE improvements. For the other systems, when the bond term is fixed, the RMSE improvements become smaller than the all-bonded-terms-flexible case, but the betterment is still not very small. When bond stretching and angle bending terms are both fixed, the GAFF2 refitting leads to negligible RMSE improvements for all ions under consideration, which suggests that the GAFF2 torsional potential is actually of satisfactory accuracy. Overall, the above detailed refitting check suggests that the improvements in refitting GAFF2 mainly come from the bond stretching term and in some cases also the angle bending term, while the torsional term is perturbed minimally, which validates our hypothesis.

As bond stretching and angle bending do not have significant influences on the formation of threedimensional structural motifs and the force-field refitting requires the generation of computationally costly $a b$ initio data, we thus wonder whether it is necessary to refit the existing GAFF2 parameters. Further, as the torsional rearrangement is actually the main influencing factor among bonded interactions determining the conformational equilibria in the three-dimensional assembly of condensed-phase systems including ionic liquids, the small errors of torsional parameters in the pre-fitted GAFF2 parameter set actually suggest that this transferable force field already contains parameters of acceptable quality and the direct application of them will not cause significant problems. We have also repeated the above procedure for GAFF, which gives very similar results (not shown). Thus, using the GAFF derivatives in the simulation of ionic liquids derivatives would not incur significant problems. However, it should be noted that although the restrained parameter adjustment requires further computational costs and leads to marginal improvements for torsional terms, doing such calculations is meaningful for two reasons. First, the quality of force-field parameters is indeed improved by adjusting existing parameters in a molecule-specific manner targeting a good high-level reference. If we include all bonded interactions in the refitting, bond stretching and angle bending motions would also be represented in a way closer to the ab initio reference, which is especially important when bond- 
length-related observables are under consideration. Second, for newly explored species, we would never know whether the pre-fitted parameter sets could produce satisfactory results until benchmark calculations are performed to calculate their deviations from some ab initio reference. Therefore, the force-field refitting workflow could be considered as a universally usable scheme for the treatment of any ion pairs. Although we can initiate our fast-growth solvation free energy calculation with these refitted parameters to pursue highly accurate descriptions, as the aim of the current work is to provide some guidelines for the selection of the charge scaling parameter in the widely used simulation regime employing GAFF derivatives that do not show significant problems in the treatment of the ion pairs under consideration, we stick to the widely used GAFF derivatives in the following nonequilibrium alchemical transformation. As the GAFF derivatives show similar behaviors in the force-field evaluation, in the following simulation only the GAFF2 parameter set is employed.

\subsection{Best Scaling Parameter from Density Matching.}

The most common way to get the best scaling parameter for atomic charges in the simulation of ionic liquids derivatives is monitoring the response of bulk properties to this variable. Among so many calculable bulk properties (e.g., viscosity and diffusion coefficient), the density of the simulated box wins the widest recognition. It is also observed that the best scaling parameters estimated from different bulk properties are somehow similar. Therefore, in the current benchmark calculation, we focus on the density of the ionic liquids and adjust the charge scaling parameter to achieve the best simulation-experiment agreement. The scanned scaling parameters include $0.5,0.6,0.7,0.8$ and 1.0 , as mentioned in the system construction section. This scanning area covers the possible range that the simulation-experiment agreement could be maximized, which ensures the identification of the best scaling factor for the density of ionic liquids.

The absolute values of the experimental mass densities for the ionic liquids ${ }^{88-91}$ under consideration are summarized in the caption of Fig. 4. It is clear that the densities of the three ionic liquids show significant differences. As the current density-matching benchmark focus on the agreement between experimental and simulated values, we focus on the deviation of the simulated density from the experimental reference. As shown in Fig. 4, the charge scaling influences the densities of all ionic liquids under consideration. For all ion pairs, the unscaled 1.0 system has the largest density, which is expected due to the stronger chargecharge interactions produced by fully charged molecules. When the atomic charges are scaled down, the system density decreases monotonically, which arises from the weaker inter-molecular electrostatic interactions and thus less compact structures formed by these charge-scaled ion pairs. Note that the slope of this decreasing behavior shows system dependence. With the decrease of the scaling parameter, the drop of 
the mass density of the [EMIM][DCA] ion pair is the slowest one among the three root-temperature ionic liquids, while the slopes of the other two curves (i.e., [EMIM][NTf2] and [EMIM][OTf]) are somehow similar.

The best experiment-simulation agreement happens at the intersection of the density-variation curve and the vertical zero-deviation reference. It is observed that although the three ionic liquids under consideration share the same cationic specie, their best charge scaling parameters differ. Therefore, it is improper to choose some pre-defined scaling parameter simply according to one specie involved in the ion pair composition. Further, the experimental densities of the three liquids follow the rank [EMIM][DCA] < $[$ EMIM] $[\mathrm{OTf}]<[\mathrm{EMIM}][\mathrm{NTf}]$, but from density estimation the best charge scaling parameters follow $[$ EMIM $][$ NTf2] $<[$ EMIM $][$ DCA $] \approx[$ EMIM $][$ OTf $]$, which suggests that the correlation between the absolute density and the charge scaling parameter is not good. Therefore, for practical systems, instead of determining the scaling parameter following some pre-defined rules, it is highly desirable to obtain the reliable answer from the scaling-density benchmark. However, although the exact solution of the charge scaling problem for a newly encountered system is unknown, some insights could indeed be summarized. From Fig. 4, it can be observed that the best scaling parameters for different ionic liquids differ, but these optimal solutions all lie somewhere between 0.6 and 0.7 . Therefore, it can be concluded that although the specific value of the best option for charge scaling from density estimation is system-dependent, choosing something in the neighborhood of $0.6 \sim 0.7$ is often half-optimal. As discussed previously, the density-scaling benchmark is quite effective to find the best charge scaling parameter for the simulation of bulk properties. However, as these bulk properties only involve solvent-solvent interactions, it is unclear whether this best scaling factor is proper for more complex situations such as the designed usage of these green solvents, the solvation of some solute molecule in ionic liquids derivatives. In this case, aside from the solvent-solvent interactions, the accurate description of solute-solvent interactions is also important. In the following parts, we would investigate the influence of the scaling factor on the calculated solvation free energy of a spectrum of solutes in the three ionic liquids.

\subsection{Solvation Free Energy from Fast-Growth Simulations.}

For each molecule, the swarm of $\lambda$-switching simulations for solute creation produces 100 independent microscopic works, which are then used to estimate the free energy difference or the solvation free energy at infinite dilution. Three commonly used estimators are employed. The first one is the exponential averaging (EXP) or the Jarzynski equation, ${ }^{92,93}$ which estimates the free energy difference by exponentially averaging 
microscopic nonequilibrium works, i.e., $\Delta A_{\text {solvation }}=-\ln \left\langle e^{-W_{\text {growth }}}\right\rangle$. Here, $\Delta A_{\text {solvation }}$ represents the dimensionless solvation free energy, $W_{\text {growth }}$ denotes the nonequilibrium work accumulated during the growth transformation, and the canonical bracket denotes averaging over the realizations initiated from the fully decoupled state. Although this estimator is asymptotically unbiased, the numerical behavior is unsatisfactory due to its poor statistical efficiency. ${ }^{94-96}$ Also, the analytical uncertainty of this estimator tends to be underestimated due to the presence of the upper bound. ${ }^{97}$ The second estimator is the slow-growth method $^{98-100}$ that approximates the free energy difference with the arithmetic mean of microscopic nonequilibrium works, i.e., $\Delta A_{\text {solvation }}=W_{a}=\left\langle W_{\text {growth }}\right\rangle$. This estimator is theoretically rigorous only when the pulling simulation is infinitely long, i.e., reversible pulling. The third estimator is the Gaussian approximated EXP (GEXP) method, which is a direct result of the truncated cumulant expansion of the EXP estimate, $\Delta A_{\text {solvation }}=\left\langle W_{\text {growth }}\right\rangle-\frac{\sigma_{W_{\text {growth }}}^{2}}{2}$, where $\sigma_{W_{\text {growth }}}^{2}$ denotes the variance of the work distribution. The GEXP estimator has better numerical behavior than the original EXP but introduces systematic bias when the microscopic works are not normally distributed. Aside from the free energy difference itself, accurate determination of the statistical uncertainty of the free energy estimate is also very important. As the analytical formula of the standard error tends to underestimate the statistical uncertainty and the situation is more severe for unidirectional perturbation, ${ }^{97}$ the bootstrapping method is used to calculate the statistical errors of the three free energy estimates. Note that for charged species, the molecule creation in PMEenabled simulations would lead to Coulombic artifacts (Wigner self energy, to be specific), which is significant especially for small simulation boxes. ${ }^{101-103}$ As the solute molecules considered in the current work are all in their neutral forms, which does not lead to net-charge variation in the alchemical transformation, no post-process charge correction term is needed.

We then start to check the free energy estimates from fast-growth alchemical transformations. Before dealing with solvation transformations in ionic liquids, we first validate our simulation protocol and the solute parametrization by checking the results in water, i.e., calculating the hydration free energies for all solutes under consideration. The first thing in free energy analysis is convergence check. For nearequilibrium pulling, the EXP and GEXP estimates are expected to be similar. Whether this phenomenon is observed is used as a criterion for convergence in the current unidirectional switching simulations. In the case that statistically significant differences are observed between the EXP and GEXP results, we lengthen the nonequilibrium transformation time by a factor of two in order to achieve a better level of convergence, 
as discussed in the last paragraph of section 2.2. An illustrative example for this validating procedure is presented in Fig. 5a, where the ordinary average, the exponential average, and the Gaussian approximated exponential average of microscopic works accumulated during the nonequilibrium growing transformation of 1,4-dioxane in water are compared. The free energy estimates obtained from both the directly sampled work distribution and the bootstrap resampled one are presented, where no identifiable difference could be observed between direct and bootstrapped estimates. This phenomenon suggests that no hysteresis problem is hindering the convergence of the free energy calculation. Thus, the simulation is expected to be converged. Another sign of convergence is the perfect agreement between the EXP and GEXP estimates, which suggests that the pulling is well within the near-equilibrium condition. Note that the ordinary average is still deviating from the other estimates, which indicates that the pulling is still performed in a nonequilibrium way. Namely, the reversible pulling condition is not met, although this will not harm when the free energy estimate is extracted from more rigorous EXP and GEXP estimators. As the EXP estimate is theoretically rigorous and its value is close to the GEXP one upon convergence, we use this estimate as the converged solvation free energy in the following discussion of simulation results.

We then turn to the solvation free energies of all solutes under consideration in water to check whether the parametrization of these molecules and the protocol of alchemical transformations are good enough to produce results in good agreement with the experimental values. The computed hydration free energies are summarized in Table S2. The correlation between the computed and experimental results is presented in Fig. $5 \mathrm{~b}$, where we can see that most of the computed values fall within the $\pm 1 \mathrm{kcal} / \mathrm{mol}$ line from the experimental reference. There are only several systems having more than $\pm 1 \mathrm{kcal} / \mathrm{mol}$ deviations and only one solute with a more than $\pm 2 \mathrm{kcal} / \mathrm{mol}$ error. Therefore, it is observed that the calculation is producing satisfactory results from the correlation plot. To assess the quality of calculations with some numerical quantities, we calculate three error metrics including the mean signed error (MSE), the mean absolute error (MAE), and the RMSE and two ranking coefficients of Kendall's rank correlation coefficient $(\tau)$ and Pearlman's predictive index (PI), the results of which are also presented in Table S2. We can see that the calculation achieves a small RMSE below $1 \mathrm{kcal} / \mathrm{mol}$, which is the normal accuracy of the hydration free energy calculation. MSE is close to zero, which suggests no systematic over- or under-estimation. MAE is also small enough $(<1 \mathrm{kcal} / \mathrm{mol})$. The ranking coefficients are both larger than 0.75 , which suggests that the consistency of the computed and experimental ranks of hydration free energies is satisfactory. Overall, the hydration free energies calculated with our modelling protocol are in good agreement with experimental values. Thus, the current model construction and sampling protocol are of sufficient reliability for solvation 
free energy calculation.

\subsection{Best Scaling Parameter for Atomic Charges from Solvation Free Energy in Ionic Liquids.}

The previous section provides sufficient evidence of the reliability of the solvation free energies in water (or hydration free energy) obtained from our fast-growth simulations. In this subsection, we summarize the results obtained under different scaling parameters of atomic charges for ionic liquids and identify the best option that minimizes the deviations from the experimental references. All computed solvation free energies are summarized in Table S3-S11.

\section{Response of solvation free energy to charge scaling: monotonic or not?}

In the mass density case, it is observed that such bulk property often shows some monotonic behavior when the scaling parameter is varied. This is a satisfactory behavior when the best charge scaling factor is pursued, as simple numerical scanning is sufficient to handle the job effectively. When we leave the bulk properties but concentrate on the solute-solvent interaction, it is thus curious to ask whether this monotonic behavior exists for the solvation free energy. For the numerical illustration of this issue, we use the first two solutes under consideration (i.e., 1,4-dioxane and 1-butanol) and the solvent [EMIM][DCA].

The solvation free energies obtained with different scaling parameters for the bulk ionic solvent are shown in Fig. 6 along with the sampled work distributions. As shown in Fig. 6a-c for the first solute 1,4dioxane, the microscopic works fall in a narrow range for all of the three scaling parameters simulated. The EXP and GEXP estimates agree well, which suggests the near-equilibrium condition of the nonequilibrium growth transformation and the resulting good convergence behavior of the alchemical simulation. The slowgrowth ordinary average $W_{\text {a }}$ of the microscopic works stays far away from the other two estimates, which indicates that the pulling simulation is irreversible. An interesting phenomenon about the ordinary average is that the difference between the ordinary average and the Gaussian estimate seems to be positively correlated with the scaling factor. As this difference is often considered as an estimate of the dissipation of the nonequilibrium process, the scaling down of atomic charges is actually reducing the dissipation. Also, as this difference is directly related to the width of the work distribution, the microscopic solvation works in charge-scaled ionizing solvents are thus distributed in a narrower range and the alchemical free energy simulation is easier to converge. Similar observation could be attained for the second example 1-butanol. Thus, we do not repeat such discussion. Bootstrap resampling is performed to get the numerical statistical uncertainty of the free energy estimates. The resulting bootstrapped work distributions are used to extract free energy differences, which are ultimately averaged to give the averaged bootstrapped estimate of the 
solvation free energy. These averaged bootstrapped estimates are plotted along with the direct ones in Fig. 6, where good agreements are observed. This observation again suggests that the hysteresis problem is negligible and thus the good convergence behavior of the current free energy calculation.

After finalizing the convergence check, we then turn to the physical aspect about the (non-)existence of the monotonic behavior. Instead of comparing the absolute solvation free energies, we still use the deviation from the experimental reference as the quantity to monitor in the scaling-parameter dependence. Still, the two solute molecules presented above are used as illustrative examples. As shown in Fig. 7, the solvation free energies of both solutes exhibit monotonic behaviors with respect to the variation of the scaling parameter. However, the free energy estimate of 1,4-dioxane increases monotonically (more positive) while the 1-butanol one decreases monotonically (more negative). This opposite behavior suggests that the response of solvation free energy to the variation of scaling parameter is solute-dependent. As there are a set of solutes with structurally diverse features under consideration, the whole set of solvation free energies would exhibit the averaged behavior of the whole solute set. This averaged response, although being the combined result of a set of monotonic components, may not be monotonic. If we jump out of the whole-set assessment but focus on the optimal scaling parameter for a given solute-solvent pair, a combinationdependent solution could be reached. When the solvation of 1,4-dioxane in [EMIM][DCA] is used as the reference, the optimal scaling parameter is close to 0.8 . When the reference is the combination of 1-butanol and [EMIM][DCA], the optimal solution is close to 0.7. Recalling that the density-derived solution for this ionic solvent presented in Fig. 4 is 0.7 , it is obvious that the solvation-free-energy-derived result could differ from the bulk-property-derived one significantly. Although this observation could be taken as some facts to guide the charge scaling of ionic solvents, it would be extremely useful if the physical reason that causes this phenomenon could be unveiled.

The solvation-free-energy-derived solution to the charge scaling problem differs from the density one, which is not unexpected. Below, we provide a more detailed investigation of this issue and provide an explanation from a theoretical point of view. Both solute-solvent and solvent-solvent interactions are affected by the scaling of the solvent charges. We first consider the variation of the bulk density when the atomic charges of ionic liquids are scaled down. When the charge scaling factor decreases from 0.8 to 0.6 , the mass density of the ionic liquids decreases monotonically (c.f., Fig. 4), which suggests looser intermolecular coordination. It is thus easier to create a cavity in this less compact bulk solvent, which is actually experienced during the switching-on simulation of the repulsive-dispersive solute-solvent interaction. Note that the vdW transformation also includes favorable solute-solvent interactions, but this cavity creation cost 
is often expected to be more sensitive to the variation of the solvent density. As the free energy cost for cavity creation is positive, larger scaling parameters would lead to more positive solute cavity works. By contrast, the electrostatic contribution to the solvation free energy is often negative (energetically favorable). A large scaling parameter would lead to stronger solute-solvent electrostatic stabilization and thus a more negative electrostatic contribution. As a result, the charge-scaling-dependent behavior of the solvation free energy in ionic liquids is triggered by the competition between electrostatic and vdW components of the solvation free energy. To provide the numerical validation of this hypothesis, we present in Fig. 8a-b the electrostatic and vdW contributions to the solvation free energies of the first two solutes in [EMIM][DCA] under different scaling parameters. These plots clearly show that when the charge scaling parameter grows from 0.6 to 0.8 , the solute-solvent electrostatic interaction becomes more negative/favorable and the vdW contribution becomes more positive (less favorable), which agrees with our hypothesis. The systemdependent behavior of the magnitudes of electrostatic and vdW responses leads to the solute-dependent response of the solvation free energy. Upon the elucidation of this behavior, another interesting question to ask is whether there is a system with similar electrostatic and vdW responses that trigger a close-to-zero change of the solvation free energy when the scaling parameter is varied. Fortunately, due to the inclusion of a broad spectrum of solute molecules, the observation of this phenomenon is possible. By scanning the whole solvation dataset for [EMIM][DCA], we do find some systems with this interesting behavior. An illustrative example to discuss is 1-propanol, the results of which are shown in Fig. 8c. For this system, the solvation free energy seems irrelevant to the charge scaling parameter. The reason for this intermediate behavior can be obviously seen from the component decomposition. The electrostatic and vdW contributions to the solvation free energy have similar response magnitudes, which leads to variation cancellation. The existence of solute-solvent pairs with various charge-scaling dependence (i.e., increasing, decreasing, or irrelevant) supports the diversity of the selected solvation dataset and thus the broad validity of the current benchmark calculations. The above observations also show that the monotonic behavior of bulk properties is no longer valid for the absolute solvation free energies of the whole solvation set. However, whether the deviation of the computed value from the experimental reference would be monotonic remains unknown, as system-dependent offsets are included in such calculation.

\section{Visual inspection of the calculation-experiment correlation as a tool for charge scaling.}

The quality of the calculated values is assessed with three error metrics including MSE, MAE, and RMSE and two ranking coefficients of $\tau$ and PI, as discussed in the hydration case previously. The illustrative examples 1,4-dioxane, 1-butanol and 1-propanol already show that the solvation free energies of 
different solutes behave differently when the degree of charge scaling is varied. As the current solvation benchmark is averaging the results of the given set of solute-solvent pairs and their responses to the charge scaling treatment, it is hard to say whether the quality metrics for the solvation free energies of a set of structurally different solutes would be monotonic with respect to the scaling parameter. Still, the option that produces the best result is pursued, and this apparent estimate is expected to vary with the chosen test set. However, as the current dataset already contains a spectrum of solute molecules and three representative ionic liquids, the result from the current benchmark test is expected to be valid for a wide range of soluteionic-liquids pairs.

The solvation free energies obtained from the current extensive modelling are summarized along with the corresponding experimental values in Table S3-11. The quality metrics calculated with these statistics are also provided. Before monitoring the variation of the quality metrics with respect to the scaling parameter, we first evaluate the charge scaling issue by visualizing the experiment-calculation correlation to obtain some preliminary picture of the influence of this charge scaling treatment. The EXP estimate is used in the current and following analyses. The results are plotted in a system-specific manner in Fig. 9a-c. For the first ionic solvent [EMIM][DCA] shown in Fig. 9a, the calculated solvation free energies with a scaling parameter of 0.6 have many outliers (more than $2 \mathrm{kcal} / \mathrm{mol}$ deviations). The experiment-calculation correlation is improved when the scaling factor is increased by 0.1 , i.e., producing the 0.7 results. Note that this value 0.7 is also the best scaling parameter derived from the previous density analysis shown in Fig. 4. Thus, the density-derived solution is reasonable to some extent. Larger improvements are observed when the scaling factor is increased further to 0.8. If we check the RMSEs calculated from the 0.7 and 0.8 sets in Table S4 and S5, we can see that the RMSE of the 0.7 set is $1.25 \mathrm{kcal} / \mathrm{mol}$, which is larger than the typical 1 $\mathrm{kcal} / \mathrm{mol}$ error for solvation free energies. By contrast, the value for the 0.8 set is $\sim 0.7 \mathrm{kcal} / \mathrm{mol}$, which falls satisfactorily within the $1 \mathrm{kcal} / \mathrm{mol}$ threshold. Thus, the scaling factor of 0.8 does seem more reasonable than the density-derived estimate 0.7. As for the second ionic liquids [EMIM][NTf2] shown in Fig. 9b, while the calculated solvation free energies with the scaling factors 0.6 and 0.7 have outliers falling outside the 2 $\mathrm{kcal} / \mathrm{mol}$ deviation line, the 0.8 -scaled results are all close to the $\mathrm{y}=\mathrm{x}$ line. Thus, from this correlation plot we can say that the value of 0.8 could lead to better performance than 0.6 and 0.7 . Note that the densityderived scaling parameter for this ionic solvent is 0.6 , which is much smaller than the current solvationcorrelation-derived 0.8. The experiment-calculation correlation for the last ionic liquids [EMIM][OTf] is presented in Fig. 9c, where we can see that all calculated results fall inside the $\pm 2 \mathrm{kcal} / \mathrm{mol}$ deviation line, irrespective of the scaling factor. The results obtained with the scaling factor of 0.6 seem to have larger 
errors/deviations than the 0.7 ones, and the 0.8 results are generally higher/larger than the 0.7 ones. However, it is difficult to determine the best solution simply from this correlation plot. Thus, for this ionic solvent, the later quantitative comparison of quality metrics seems necessary.

Another useful experiment-calculation correlation is putting the results of all ionic liquids obtained under the same scaling parameter together, as shown in Fig. 9d-f. This comparison considers all systems under simulation as a whole set of solute-ionic-liquids pairs, and can be used to deduce a solution generally applicable to a broad range of ionic liquids rather than a specific combination (e.g., those shown in Fig. 9ac). For the scaling factor of 0.6, the results of all ionic liquids are presented in Fig. 9d. We can see that under this scaling parameter the calculated results for [EMIM][DCA] have many outliers with more than 2 $\mathrm{kcal} / \mathrm{mol}$ deviations from the experimental reference, for [EMIM][NTf2] there are only several points lying outside the $\pm 2 \mathrm{kcal} / \mathrm{mol}$ line, and for [EMIM][OTf] there is no outlier. Considering the solvation behaviors of all ionic liquids, this 0.6 scaling parameter inevitably produces significant deviations $(>2 \mathrm{kcal} / \mathrm{mol})$ from experiments. The solvation free energies in all ionic liquids obtained under the scaling factor 0.7 are presented in Fig. 9e, where a similar phenomenon is observed. Namely, the number of outliers is quite large for [EMIM][DCA], only several points are falling outside the $2 \mathrm{kcal} / \mathrm{mol}$ deviation line for [EMIM][NTf2], and no outlier is observed for [EMIM][OTf]. When the scaling factor is increased further to 0.8 , the results shown in Fig. 9f are obtained. Under this scaling parameter, interestingly, all solvation free energies in [EMIM][DCA] are within the $\pm 2 \mathrm{kcal} / \mathrm{mol}$ line, which suggests the applicability of this scaling parameter for this solvent. For the other two ionizing solvents, the number of outliers is also zero. Therefore, this 0.8 scaling parameter is considered to be generally applicable to ionic liquids with different compositions. It should be noted that for all of the three ionic liquids under simulation, the density-derived solutions never reach such a high value, which suggests that the solvation-derived estimate with across-the-broad applicability could differ drastically from the density-derived estimates. This phenomenon is not unexpected, as the accurate description of the solvation process requires accurate and balanced solutesolvent and solvent-solvent interactions, while only the latter (i.e., the solvent-solvent interaction) is taken into consideration in the bulk-property-derived estimate.

\section{Quality metrics as the thermodynamic criteria for charge scaling.}

To determine the best scaling parameter directly from the quality metrics, we present the dependences of three error metrics (RMSE, MSE and MAE) and two ranking coefficients (Kendall $\tau$ and PI) on the scaling factor for atomic charges in Fig. 10. For the first ionizing solvent [EMIM][DCA], as shown in Fig. $10 \mathrm{a}$, all of the three error metrics exhibit a monotonically decreasing behavior with respect to the scaling 
parameter. The positive MSE indicates systematic over- or under-estimation (depending the sign considered in discussion). This systematic deviation gradually vanishes with increased scaling parameters. RMSE achieves its smallest value when the scaling factor is 0.8 and is well below the $1 \mathrm{kcal} / \mathrm{mol}$ threshold that is the normally observed mean error for hydration free energy. Therefore, the best scaling parameter derived from error metrics is 0.8 for this solvent. As for the ranking coefficients presented in Fig. 10b, monotonically increasing behaviors are observed for both metrics. The highest value of Kendall $\tau$ is 0.69 , and that of PI is 0.85 . These high ranking coefficients suggest good agreements between computed and experimental ranks of solvation free energies. Thus, the best scaling parameter derived from ranking coefficients is 0.8 . Taken together, the best scaling parameters determined from error metrics and ranking coefficients agree well and are 0.8 . Note that this solvation-thermodynamics-derived 0.8 solution is 0.1 larger than the density-derived estimate shown previously. Another worth noting point is that we cannot guarantee that the minimum error point is reached in the scanned range of the scaling parameter, mainly due to the monotonic behaviors of all quality metrics. A possible observation is further lower errors and better ranks for the scaling parameter of 0.9 . However, as this 0.8 scaling factor simultaneously ensures accurate calculation of solvation free energies and maintains a good level of density reproduction, we can conclude that this value is a half-optimal option at least.

For the second ionic liquids [EMIM][NTf2], the error metrics and the ranking coefficients are presented in Fig. 10c-d. Interestingly, for the second solvent the error metrics calculated with the 0.6 and 0.7 results are similar, although quality metrics produced by the 0.7 set are still superior than the 0.6 case. Note that the RMSE calculated with the 0.7 set is already below the $1 \mathrm{kcal} / \mathrm{mol}$ threshold for solvation free energy, which suggests that this scaling parameter is able to produce solvation thermodynamics with acceptable accuracy. When it comes to the ranking coefficients, a modest improvement could still be observed. When the scaling factor increases further to 0.8, all error metrics experience a drastic fall. The RMSE achieves $0.45 \mathrm{kcal} / \mathrm{mol}$, which indicates an extreme accuracy of the solvation free energy calculation. MAE exhibits a behavior similar to RMSE. The MSE of $-0.03 \mathrm{kcal} / \mathrm{mol}$ suggests no systematic over- or under-estimation. Significant improvements are also observed for ranking coefficients. Therefore, the best scaling parameter determined from extensive solvation free energy calculations for this ionic solvent [EMIM][NTf2] is 0.8 . Note that this value is 0.2 larger than the density-derived solution 0.6 , which again suggests the inappropriateness of using bulk properties as reference observables when pursuing accurate solvation behaviors that involve both solute-solvent and solvent-solvent interactions.

The results for the third ionic solvent [EMIM][OTf] are shown in Fig. 10e-f. The error metrics in Fig. 
10e suggest that the scaling factors of 0.7 and 0.8 produce similar performances in the solvation set and their RMSEs are below the $1 \mathrm{kcal} / \mathrm{mol}$ threshold. Thus, both scaling factors seem acceptable from error metrics. By contrast, the scaling factor of 0.6 is obviously worse and should not be considered in practical simulations. Interestingly, unlike the scaling-factor-irrelevant error metrics in the range of $0.7-0.8$, both ranking coefficients shown in Fig. 10f exhibit monotonically increasing behaviors with respect to the scaling parameter in the whole scanned range $0.6-0.8$. Thus, we can conclude that the value of 0.8 is better than 0.7 and the best scaling factor for this solvent [EMIM][OTf] is 0.8. By comparing this solvation-free-energyderived solution with the density-derived one shown previously, we still observe that the recommended value from the solvation set is 0.1 larger than the bulk-property-derived estimate. Considering the consistent solvation-larger-than-density behavior observed for all of the three ionic liquids, we summarize that the best solution determined from solvation thermodynamics is generally slightly larger $(\sim 0.1)$ than the bulkproperty-derived one. Based on this fact, it is possible to devise a feasible way to obtain the best scaling parameter that produces balanced solute-solvent and solvent-solvent interactions. As large-scale solvation free energy calculations are computationally demanding and should be avoided in practical simulations, we recommend scanning the density-scaling-parameter profile exhaustively to find the best density-derived solution and then adding $\sim 0.1$ to approximate the solvation-thermodynamics-derived scaling parameter.

Although the above ion-pair-specific benchmark provides valuable thermodynamic insights about the charge scaling problem, in the last solvation benchmark we consider all ionic liquids as a whole and expect to determine a universal scaling factor applicable for a series of ion-pair combinations. All solvation free energies in the three ionic liquids are combined to produce a total solvation set, from which error metrics and ranking coefficients are computed. As shown in Fig. 10g-h, the error metrics and the ranking coefficients show consistent monotonic behaviors with respect to the scaling parameter, and the calculation achieves its highest performance at the scaling parameter 0.8 . This value is at least 0.1 larger than any density-derived estimates for individual ionic solvent forming the total set. Therefore, from this total benchmark we can also conclude that the solvation-free-energy-derived scaling parameter is slightly higher than the density-derived one.

\subsection{The Partition Benchmark.}

The above computational perspective about the solvation-free-energy-derived scaling parameter is extremely useful when accurate solvation behaviors are pursued. The inter-molecular coordination between the ionic liquids derivatives and the external agent would be described very well. Although the absolute 
solvation free energy is a critical criterion with significant thermodynamic relevance for practical chemical and biological applications, another crucial thermodynamic observable highly related to it could also be derived from the statistics accumulated in this work as another criterion for the charge-scaling issue. The basic idea is combining the computational results from the solute-in-ionic-liquids and the solute-in-water cases to produce the relative solvation free energy of each external agent in the two solvents, which is often called as the transfer free energy used to estimate the water-ionic-liquids partition coefficient. Although the logarithm of the partition coefficient $\log$ P defined as the transfer free energy divided by RT $\ln 10$ is the observable commonly used in this solute-distribution situation, as this suppressed quantity is smaller in value than the free energy difference itself at physiological conditions, we stick to the transfer free energy that is more numerically sensitive in the partition benchmark, similar to our previous treatment of $\mathrm{pKa}$ shifts. ${ }^{104}$ The accuracy of the calculation of this relative free energy depends on both the solute-water description and the solute-ionic-liquids counterpart. Therefore, aside from the correct description of solvation thermodynamics in ionic liquids derivatives evaluated in the previous case, the modelling accuracy of the solvation in water is also an influencing part. Here, as the popular TIP3P model is used to describe water molecules, any observation about the partition behavior from the current computational perspective would be limited to this model, although the hydration thermodynamics produced by popular water models are often similar.

In this partition benchmark, the water-ionic-liquids transfer free energy for each solute is calculated as the difference between its solvation free energies in these two solvents. As these solvation data are already available in Table S2-S11, we do not duplicate the exact values of transfer free energies but focus on quality metrics directly. The error metrics and the ranking coefficients for the water-[EMIM][DCA] partition set obtained with different scaling parameters are presented in Fig. 11a-b. Interestingly, compared with the previous [EMIM][DCA] solvation benchmark, although the error metrics still show monotonic behaviors with respect to the scaling parameter, the ranking coefficients seem unchanged for scaling factors in the range of 0.6 and 0.8 , which obviously is caused by the inclusion of the calculated hydration free energies. This phenomenon clearly shows that the partition benchmark is not equivalent to the solvation set. It is worth noting that the absolute values of ranking coefficients are enlarged when including hydration free energies in the calculation. For instance, PI computed from solvation free energies in the ionic solvent [EMIM][DCA] is $\sim 0.6$ when the scaling factor is 0.6 , and reaches a value of $\sim 0.8$ when the scaling parameter is increased to 0.8 . However, in the partition benchmark, the values of PI under all scaling parameters are larger than 0.8 . This enlargement due to the inclusion of hydration free energies is obviously 
caused by the cancellation of errors in the calculated solvation free energies in the two solvents for individual solute. It is hard to define the best scaling parameter from the ranking coefficients as the three estimates (i.e., with scaling factors $0.6,0.7$ and 0.8 ) are extremely similar, but we can still conclude that the best option is 0.8 according to the error metrics. Therefore, the basic conclusion about charge scaling remains the same as the solvation set for this ionic solvent [EMIM][DCA].

For the second ionic solvent [EMIM][NTf2], the monotonic behavior for the error metrics observed in the solvation set vanishes in the current partition benchmark, although the scaling parameter of 0.8 still shows minor superiority among the three values, as shown in Fig. 11c. Further, in Fig. 11d, the monotonically increasing behaviors of ranking coefficients observed in the solvation set no longer exist. Instead, the opposite behavior (i.e., monotonically decreasing behavior) is observed in the water[EMIM][NTf2] partition set. Therefore, the best scaling parameter determined from ranking coefficients is 0.6. The disagreement between recommended values from error estimation and ranking analysis makes the determination of the best scaling parameter a difficult thing for the partition benchmark. If the smallest errors/deviations are pursued, the scaling factor of 0.8 should be chosen. On the other hand, if the ranks of transfer free energies (partition coefficients) are of utmost importance, the value of 0.6 is recommended. Anyway, as this partition set also involves the errors introduced by hydration free energies in TIP3P water, the above observations and the resulting recommendations are limited to the TIP3P-ionic-liquids combination. When a different water model is employed, different conclusions are expected to be reached. Another worth noting point is still the significant improvement of the absolute values of ranking coefficients. Similar to the previous water-[EMIM][DCA] case, the values of $\tau$ and PI in the solvation set are significantly smaller than those in the partition benchmark. This phenomenon, obviously, arises from the error cancellation for the calculated solvation free energies of the same solute in water and in ionic liquids.

As for the third ionic solvent [EMIM][OTf], the error metrics and the ranking coefficients for the water[EMIM][OTf] partition set are shown in Fig. 11e-f. The scaling-parameter dependence of the error metrics in the partition set differs from the [EMIM][OTf] solvation case, where the error metrics RMSE and MAE obtained from the scaling factors 0.7 and 0.8 are similar and are obviously smaller than the 0.6 results. For the partition set, RMSE and MAE reach their minimum values when the scaling factor is 0.7 . As for the ranking coefficients, monotonically decreasing behaviors are observed and the best scaling parameter is found to be 0.6 . The best solutions determined from error estimation and rank analysis again do not agree well, which makes it difficult to determine the best scaling factor. However, it can be concluded that the best solution is expected to fall in the range $0.6 \sim 0.7$, which overlaps with the $0.7 \sim 0.8$ option derived from the 
solvation set to some extent. Note that the improvement of the absolute values of ranking coefficients could still be observed in this case. For instance, the largest PI observed in the [EMIM][OTf] solvation case is smaller than 0.9 , but the values of PI under all of the three scaling parameters in the water-[EMIM][OTf] partition set are well above the 0.9 line.

Overall, considering the above observations in the partition benchmark, it can be concluded that the solution to the charge scaling problem obtained from partition coefficients or transfer free energies shows some deviations from the solvation-derived estimate, and the magnitude of this deviation depends on the employed water model and also the solvent set itself. In some cases, the deviation is minimal and the partition benchmark gives exactly the same recommendation as the solvation set, while in cases that the deviation is large the conclusions obtained from transfer and solvation free energies differ significantly. The last part of the partition benchmark is merging the above ion-pair-specific benchmarks into a single 'total' set, which is used to determine a universal scaling factor applicable for a series of water-ionic-liquids combinations. The drastically different behaviors of the three water-ionic-liquids partition sets observed above are averaged/combined, and the resulting error metrics and ranking coefficients of this total partition set are shown in Fig. 11g-h. Interestingly, although the three water-ionic-liquids components behave differently, the total set produces error metrics and ranking coefficients with consistent monotonic behaviors with respect to the scaling parameter. The best option from this total partition set can be straightforwardly identified to be 0.8 , which is in perfect agreement with the total solvation set reported in the previous section about solvation thermodynamics. As the best scaling parameter obtained from the solvation set is generally slightly higher than the density-derived estimate, from the current partition benchmark we can conclude that the water-ionic-liquids partition-coefficients-derived (or equivalently transfer-free-energy-derived) scaling parameter is slightly higher than the density-derived one.

\section{Conclusion}

Novel functionalized solvents are developed as replacements for conventional organic volatile, toxic and flammable solvents. Ionized species are considered as promising candidates for achieving this goal. Ionic liquids and their more recent derivative deep eutectic solvents are receiving attentions in recent years. These tunable functionalized solvents exhibit satisfactory solvation power for organic and metallic species. Current applications can be observed in organic synthesis, electrochemical studies, enzyme-catalyzed reactions, liquid-liquid extraction and chromatography. Molecular simulations are becoming increasingly popular in the investigation of the dynamic and thermodynamic behaviors of these novel solvents. 
Classical force fields are widely applied due to their simplicity and the resulting computational efficiency. The basic ingredients of all-atom force fields include bonded and non-bonded terms, with the former determining the intra-molecular conformational preference and the latter defining the strength and pattern of inter-molecular coordination. The widely accepted treatment of these interactions is the combination of the transferable GAFF derivatives and the RESP charge scheme, with the former defining the intra-molecular bonded interactions and the vdW component of inter-molecular interactions and the latter determining the most influencing part of inter-molecular coordination, the electrostatics. Despite the massive use of this combined scheme in simulations of ionic liquids derivatives, the quality of the modelling regime is seldom validated. Therefore, a thorough evaluation of the pre-fitted parameters in the GAFF derivatives is presented in this work. To identify the problematic parts of these transferable parameters, we further present a component-specific refitting procedure to separate the errors introduced by bond stretching, angle bending, and dihedral/torsional terms. The detailed results obtained from our evaluation and refitting suggest that the bond stretching term is actually the most problematic part of GAFF derivatives. In some cases, the angle bending term also contributes some errors. Interestingly, the more influencing part for intramolecular conformational arrangement, the dihedral/torsional potential, is found to perform quite well for ionic liquids. Thus, it can be concluded that in the case that bond stretching and angle bending motions are not important, the GAFF derivatives are actually providing a very good description of the energetics of ionic liquids. However, when these stiff degrees of freedom do matter, refitting these existing parameter sets is necessary.

Further, it is widely believed that the fixed-charge treatment of electrostatics generally fails to reproduce the condensed-phase behavior of these green solvents due to charge transfer and polarization induced by environmental coupling. To circumvent the electrostatic problem, uniform scaling of atomic charges is often applied. The scaling parameter for atomic charges is derived mainly in the simulation of a bulk solvent box. The best solution is achieved when the experiment-simulation agreement is maximized. A bulk property widely used in the mass density of ionic liquids derivatives. However, in the investigation of the most common and designed usage of these green solvents, i.e., the solvation process of some selected molecules in ionic liquids derivatives, the thermodynamic observable with the highest relevance is, actually, the solvation free energy. Although the bulk-property-derived charge scaling parameter could be effective in describing the solvent-solvent interaction, it may produce unbalanced descriptions of solute-solvent and solvent-solvent interactions, which leads to wrong thermodynamic behaviors in the solvation process. To obtain some guidelines of charge scaling from this commonly overlooked solvation thermodynamic 
perspective, we perform nonequilibrium free energy calculations to obtain the solvation free energies of a spectrum of molecules in three ionic liquids. The pleasantly parallel nonequilibrium switching simulations are performed for solute-solvent pairs under different scaling factors for atomic charges. A worth noting observation is that although the bulk property of density shows monotonic behavior with respect to the scaling parameter, the solvation free energies do not exhibit consistent behavior. For some solutes, the solvation free energy increases monotonically when the scaling parameter is increased, while for some solutes the opposite behavior is observed. However, we also observe cases that have solvation free energies irrelevant to the charge scaling factor. The underlying physics of this inconsistent scaling-factor dependence is found to be the competing electrostatic and $\mathrm{vdW}$ responses to the variation of the scaling parameter. When the scaling factor increases, the favorable electrostatic interaction is strengthened, while the vdW contribution becomes less favorable due to increased solute cavity work. As a result, different solute-solvent pairs show different responses to the charge scaling parameter and the behavior of the whole solvation set is actually averaging over a large set of solvation components. The apparent solution of the charge scaling issue is obtained by monitoring the scaling-factor dependence of three error metrics and two ranking coefficients. When the error metrics are minimized and the ranking coefficients are maximized, the experiment-calculation agreement achieves the highest level and the optimal charge scaling parameter is obtained. Intriguingly, the best scaling parameter derived from solvation thermodynamics differs from the bulk-property-derived one. Generally, although the density-derived best scaling parameters for the three ionic liquids studied in this work fall in the range of 0.6 0.7, the optimal solutions obtained from solvation free energies are often 0.1 larger, which leads to the range of $0.7 \sim 0.8$. As only the neighborhood of the best density-derived estimates is scanned in the solvation free energy calculation, we cannot omit the possibility that better results can be obtained by further increasing the scaling parameter (e.g., to 0.9). However, as the current recommendation of $0.7 \sim 0.8$ ( 0.1 larger than the density-derived estimate, to be specific) is able to produce solvation free energies in good agreement with the experimental reference $(\mathrm{RMSE}<1 \mathrm{kcal} / \mathrm{mol}$ which is the typical error of hydration free energies) and produce a mass density close to the experimental value, we believe that the current scanning suffices for the modelling of ionic liquids derivatives with classical fixed-charge force fields. Based on the phenomenon that the density-derived scaling factor is generally $\sim 0.1$ smaller than the solvation-derived one, we propose a computationally feasible way to obtain the best scaling parameter that produces balanced solute-solvent and solvent-solvent interactions. Namely, we first scan the density-scaling-parameter profile to get the density-derived solution and then add $\sim 0.1$ to get an approximate solvation-derived estimate. Another thermodynamic perspective is obtained by 
combining the solvation free energies in ionic liquids and in water. Taking their differences leads to an estimate of the transfer free energy of solutes between these two solvents, which is highly related to the partition coefficient. Although this partition benchmark provides a similar picture of the charge scaling issue, in many aspects different behaviors of the thermodynamic parameters are observed. Overall, the obtained insights about the component-wise force-field quality and the thermodynamic perspectives of the charge scaling issue provide useful guidelines for molecular modelling of ionic liquids derivatives.

\section{Acknowledgement}

This work was supported by the National Natural Science Foundation of China (Grant No. 21633001). Part of the simulation was performed on the high-performance computing platform of the Center for Life Science (Peking University). We thank Dr. Zhe Huai (XtalPi) and the anonymous reviewers for valuable comments and critical reading.

\section{Conflict of Interest Statement}

There are no conflicts of interest to declare.

\section{Supporting Information Description}

The time series of the deviation of the MM (GAFF2 and the refitted parameter set) energetics from the $a b$ initio B97-3c reference for all ions, a summary of the solutes considered in the current work, the results of hydration free energies of all solutes, and the solvation free energies in the three ionic liquids under the scaling parameters $0.6,0.7$ and 0.8 are provided in the supporting material.

\section{Data and Software Availability}

The data that support the findings of this study are available from the corresponding author upon reasonable request. 


\section{References}

1. Romero, A.; Santos, A.; Tojo, J.; Rodríguez, A., Toxicity and biodegradability of imidazolium ionic liquids. Journal of hazardous materials 2008, 151, 268-273.

2. Martín-Calero, A.; Pino, V.; Afonso, A. M., lonic liquids as a tool for determination of metals and organic compounds in food analysis. TrAC, Trends Anal. Chem. 2011, 30, 1598-1619.

3. Abbott, A. P.; Capper, G.; Davies, D. L.; McKenzie, K. J.; Obi, S. U., Solubility of metal oxides in deep eutectic solvents based on choline chloride. Journal of Chemical \& Engineering Data 2006, 51, 1280-1282.

4. Arce, A.; Earle, M. J.; Rodríguez, H.; Seddon, K. R., Separation of aromatic hydrocarbons from alkanes using the ionic liquid 1-ethyl-3-methylimidazolium bis \{(trifluoromethyl) sulfonyl\} amide. Green Chemistry 2007, 9, 70-74.

5. Selvan, M. S.; McKinley, M. D.; Dubois, R. H.; Atwood, J. L., Liquid - liquid equilibria for toluene+ heptane+ 1-ethyl3-methylimidazolium triiodide and toluene+ heptane+ 1-butyl-3-methylimidazolium triiodide. Journal of Chemical \& Engineering Data 2000, 45, 841-845.

6. Jacquemin, J.; Husson, P.; Majer, V.; Gomes, M. F. C., Influence of the cation on the solubility of CO 2 and $\mathrm{H} 2$ in ionic liquids based on the bis (trifluoromethylsulfonyl) imide anion. J. Solution Chem. 2007, 36, 967-979.

7. Whitehead, J. A.; Lawrance, G. A.; McCluskey, A., 'Green'leaching: recyclable and selective leaching of gold-bearing ore in an ionic liquid. Green Chemistry 2004, 6, 313-315.

8. Zhang, M.; Kamavaram, V.; Reddy, R. G., Aluminum electrowinning in ionic liquids at low temperature. Light Metals 2005, 2005, 583-588.

9. Łachwa, J.; Morgado, P.; Esperanca, J. M.; Guedes, H. J.; Canongia Lopes, J. N.; Rebelo, L. P. N., Fluid-phase behavior of $\{1$-hexyl-3-methylimidazolium bis (trifluoromethylsulfonyl) imide,[C6mim][NTf2],+ C2- C8 n-alcohol\} mixtures: Liquid

- liquid equilibrium and excess volumes. Journal of Chemical \& Engineering Data 2006, 51, 2215-2221.

10. Verma, R.; Mohan, M.; Goud, V. V.; Banerjee, T., Operational Strategies and Comprehensive Evaluation of Menthol Based Deep Eutectic Solvent for the Extraction of Lower Alcohols from Aqueous Media. ACS Sustainable Chemistry \& Engineering 2018, 6, 16920-16932.

11. Chagnoleau, J.-B.; Papaiconomou, N.; Jamali, M.; Abranches, D. O.; Coutinho, J. A. P.; Fernandez, X.; Michel, T., Toward a Critical Evaluation of DES-Based Organic Biphasic Systems: Are Deep Eutectic Solvents so Critical? ACS Sustainable Chemistry \& Engineering 2021, 9, 9707-9716.

12. van Osch, D. J. G. P.; Dietz, C. H. J. T.; Warrag, S. E. E.; Kroon, M. C., The Curious Case of Hydrophobic Deep Eutectic Solvents: A Story on the Discovery, Design, and Applications. ACS Sustainable Chemistry \& Engineering 2020, 8, 1059110612.

13. Domańska, U.; Królikowska, M.; Królikowski, M., Phase behaviour and physico-chemical properties of the binary systems $\{1$-ethyl-3-methylimidazolium thiocyanate, or 1-ethyl-3-methylimidazolium tosylate+ water, or + an alcohol\}. Fluid Phase Equilib. 2010, 294, 72-83.

14. Kareem, M. A.; Mjalli, F. S.; Hashim, M. A.; Hadj-Kali, M. K.; Bagh, F. S. G.; Alnashef, I. M., Phase equilibria of toluene/heptane with tetrabutylphosphonium bromide based deep eutectic solvents for the potential use in the separation of aromatics from naphtha. Fluid Phase Equilib. 2012, 333, 47-54.

15. Habibi, E.; Ghanemi, K.; Fallah-Mehrjardi, M.; Dadolahi-Sohrab, A., A novel digestion method based on a choline chloride-oxalic acid deep eutectic solvent for determining Cu, Fe, and $\mathrm{Zn}$ in fish samples. Analytica chimica acta 2013, 762, 61-67.

16. Hayyan, A.; Hashim, M. A.; Hayyan, M.; Mjalli, F. S.; AlNashef, I. M., A novel ammonium based eutectic solvent for the treatment of free fatty acid and synthesis of biodiesel fuel. Industrial Crops and Products 2013, 46, 392-398.

17. Sapir, L.; Stanley, C. B.; Harries, D., Properties of polyvinylpyrrolidone in a deep eutectic solvent. J. Phys. Chem. A 2016, 120, 3253-3259. 
18. García-Alvarez-Coque, M.; Ruiz-Angel, M. J.; Berthod, A.; Carda-Broch, S., On the use of ionic liquids as mobile phase additives in high-performance liquid chromatography. A review. Analytica chimica acta 2015, 883, 1-21.

19. Xiaohua, X.; Liang, Z.; Xia, L.; Shengxiang, J., lonic liquids as additives in high performance liquid chromatography: Analysis of amines and the interaction mechanism of ionic liquids. Analytica chimica acta 2004, 519, $207-211$.

20. Anderson, J. L.; Armstrong, D. W., High-stability ionic liquids. A new class of stationary phases for gas chromatography. Analytical Chemistry 2003, 75, 4851-4858.

21. Marszałł, M. P.; Kaliszan, R., Application of ionic liquids in liquid chromatography. Critical Reviews in Analytical Chemistry 2007, 37, 127-140.

22. Sun, Z.; Liu, Z., BAR-Based Multi-Dimensional Nonequilibrium Pulling for Indirect Construction of QM/MM Free Energy Landscapes: Varying the QM Region. Adv. Theory Simul. 2021, 2100185.

23. Sun, Z.; Zhang, J. Z. H., Thermodynamic Insights of Base Flipping in TNA Duplex: Force Fields, Salt Concentrations, and Free-Energy Simulation Methods. CCS Chemistry 2021, 3, 1026-1039.

24. Sun, Z.; Huai, Z.; He, Q.; Liu, Z., A General Picture of Cucurbit [8] uril Host-Guest Binding. J. Chem. Inf. Model. 2021.

25. Sun, Z., SAMPL7 TrimerTrip Host-Guest Binding Poses and Binding Affinities from Spherical-Coordinates-Biased Simulations. J. Comput.-Aided Mol. Des. 2021, 35, 105-115.

26. Sun, Z., BAR-based multi-dimensional nonequilibrium pulling for indirect construction of QM/MM free energy landscapes: from semi-empirical to ab initio. Phys. Chem. Chem. Phys. 2019, 21, 21942-21959

27. Sun, Z.; Wang, X.; Zhang, J. Z. H.; He, Q., Sulfur-substitution-induced base flipping in the DNA duplex. Phys. Chem. Chem. Phys. 2019, 21, 14923-14940.

28. Huai, Z.; Sun, Z., Titration of Adenine in a GA mismatch with Grand Canonical Simulations. Journal of Computational Biophysics and Chemistry 2020, 20, 165-173.

29. Gao, Y.; Zhu, T.; Chen, J., Exploring drug-resistant mechanisms of I84V mutation in HIV-1 protease toward different inhibitors by thermodynamics integration and solvated interaction energy method. Chem. Phys. Lett. 2018, 706, 400-408. 30. Chen, J.; Zhang, S.; Wang, W.; Pang, L.; Zhang, Q.; Liu, X., Mutation-Induced Impacts on the Switch Transformations of the GDP- and GTP-Bound K-Ras: Insights from Multiple Replica Gaussian Accelerated Molecular Dynamics and Free Energy Analysis. J. Chem. Inf. Model. 2021, 61, 1954-1969.

31. Boz, E.; Stein, M., Accurate Receptor-Ligand Binding Free Energies from Fast QM Conformational Chemical Space Sampling. International Journal of Molecular Sciences 2021, 22, 3078.

32. Köddermann, T.; Paschek, D.; Ludwig, R., Molecular Dynamic Simulations of Ionic Liquids: A Reliable Description of Structure, Thermodynamics and Dynamics. ChemPhysChem 2007, 8, 2464-2470.

33. Perlt, E.; Ray, P.; Hansen, A.; Malberg, F.; Grimme, S.; Kirchner, B., Finding the best density functional approximation to describe interaction energies and structures of ionic liquids in molecular dynamics studies. J. Chem. Phys. 2018, 148, 193835.

34. Yasaka, Y.; Klein, M. L.; Nakahara, M.; Matubayasi, N., Exploring the reorientation of benzene in an ionic liquid via molecular dynamics: Effect of temperature and solvent effective charge on the slow dynamics. J. Chem. Phys. 2011, 134, 191101.

35. Zhang, Y.; Maginn, E. J., A Simple AIMD Approach to Derive Atomic Charges for Condensed Phase Simulation of Ionic Liquids. J. Phys. Chem. B 2012, 116, 10036-10048.

36. Chaban, V., Polarizability versus mobility: atomistic force field for ionic liquids. Phys. Chem. Chem. Phys. 2011, 13, 16055-16062.

37. Chaban, V. V.; Prezhdo, O. V., A new force field model of 1-butyl-3-methylimidazolium tetrafluoroborate ionic liquid and acetonitrile mixtures. Phys. Chem. Chem. Phys. 2011, 13, 19345-19354.

38. Zhang, Y.; Maginn, E. J., The effect of C2 substitution on melting point and liquid phase dynamics of imidazolium based-ionic liquids: insights from molecular dynamics simulations. Phys. Chem. Chem. Phys. 2012, 14, 12157-12164. 39. Maple, J. R.; Dinur, U.; Hagler, A. T., Derivation of force fields for molecular mechanics and dynamics from ab initio energy surfaces. Proc. Natl. Acad. Sci. USA 1988, 85, 5350-5354.

40. Allinger, N. L.; Chen, K.; Lii, J. H., An improved force field (MM4) for saturated hydrocarbons. J. Comput. Chem. 1996, $31 / 70$ 
17, 642-668.

41. Halgren, T. A.; Nachbar, R. B., Merck molecular force field. IV. Conformational energies and geometries for MMFF94. J. Comput. Chem. 1996, 17, 587-615.

42. Wang, J.; Cieplak, P.; Kollman, P. A., How well does a restrained electrostatic potential (RESP) model perform in calculating conformational energies of organic and biological molecules? J. Comput. Chem. 2000, 21, 1049-1074.

43. Bixon, M.; Lifson, S., Potential functions and conformations in cycloalkanes. Tetrahedron 1967, 23, 769-784.

44. Aleman, C.; Orozco, M.; Luque, F., Multicentric charges for the accurate representation of electrostatic interactions in force-field calculations for small molecules. Chem. Phys. 1994, 189, 573-584.

45. Vahid, A.; Maginn, E. J., Monte Carlo simulation and SAFT modeling study of the solvation thermodynamics of dimethylformamide, dimethylsulfoxide, ethanol and 1-propanol in the ionic liquid trimethylbutylammonium bis(trifluoromethylsulfonyl)imide. Phys. Chem. Chem. Phys. 2015, 17, 7449-7462.

46. Mecklenfeld, A.; Raabe, G., Comparison of RESP and IPolQ-mod partial charges for solvation free energy calculations of various solute/solvent pairs. J. Chem. Theory Comput. 2017, 13, 6266-6274.

47. Mohamed, N. A.; Bradshaw, R. T.; Essex, J. W., Evaluation of solvation free energies for small molecules with the AMOEBA polarizable force field. J. Comput. Chem. 2016, 37, 2749-2758.

48. Muddana, H. S.; Sapra, N. V.; Fenley, A. T.; Gilson, M. K., The SAMPL4 hydration challenge: evaluation of partial charge sets with explicit-water molecular dynamics simulations. J. Comput.-Aided Mol. Des. 2014, 28, 277-287.

49. Mobley, D. L.; Dumont, E.; Chodera, J. D.; Dill, K. A., Comparison of charge models for fixed-charge force fields: small-molecule hydration free energies in explicit solvent. J. Phys. Chem. B 2007, 111, 2242-2254.

50. Vassetti, D.; Pagliai, M.; Procacci, P., Assessment of GAFF2 and OPLS-AA general force fields in combination with the water models TIP3P, SPCE, and OPC3 for the solvation free energy of druglike organic molecules. J. Chem. Theory Comput. 2019, 15, 1983-1995.

51. Sitkoff, D.; Sharp, K. A.; Honig, B., Accurate calculation of hydration free energies using macroscopic solvent models. J. Phys. Chem. 1994, 98, 1978-1988.

52. Acree, J., William E; Abraham, M. H., The analysis of solvation in ionic liquids and organic solvents using the Abraham linear free energy relationship. Journal of Chemical Technology \& Biotechnology: International Research in Process, Environmental \& Clean Technology 2006, 81, 1441-1446.

53. Tunon, I.; Silla, E.; Pascual-Ahuir, J., Continuum-uniform approach calculations of the solubility of hydrocarbons in water. Chem. Phys. Lett. 1993, 203, 289-294.

54. Sprunger, L. M.; Proctor, A.; Acree Jr, W. E.; Abraham, M., LFER correlations for room temperature ionic liquids: Separation of equation coefficients into individual cation-specific and anion-specific contributions. Fluid Phase Equilib. 2008, 265, 104-111.

55. Mutelet, F.; Revelli, A. -L.; Jaubert, J.-N.; Sprunger, L. M.; Acree Jr, W. E.; Baker, G. A., Partition coefficients of organic compounds in new imidazolium and tetralkylammonium based ionic liquids using inverse gas chromatography. Journal of Chemical \& Engineering Data 2010, 55, 234-242.

56. Revelli, A.-L.; Mutelet, F.; Jaubert, J.-N., Prediction of Partition Coefficients of Organic Compounds in lonic Liquids: Use of a Linear Solvation Energy Relationship with Parameters Calculated through a Group Contribution Method. Industrial \& Engineering Chemistry Research 2010, 49, 3883-3892.

57. Gallicchio, E.; Zhang, L. Y.; Levy, R. M., The SGB/NP hydration free energy model based on the surface generalized born solvent reaction field and novel nonpolar hydration free energy estimators. J. Comput. Chem. 2002, 23, 517-529.

58. Marenich, A. V.; Cramer, C. J.; Truhlar, D. G., Universal solvation model based on the generalized born approximation with asymmetric descreening. J. Chem. Theory Comput. 2009, 5, 2447-2464.

59. Michielan, L.; Bacilieri, M.; Kaseda, C.; Moro, S., Prediction of the aqueous solvation free energy of organic compounds by using autocorrelation of molecular electrostatic potential surface properties combined with response surface analysis. Biorg. Med. Chem. 2008, 16, 5733-5742.

60. Bayly, C. I.; Cieplak, P.; Cornell, W.; Kollman, P. A., A well-behaved electrostatic potential based method using charge restraints for deriving atomic charges: the RESP model. J. Phys. Chem. 1992, 97, 10269-10280. 
61. Wang, J.; Wolf, R. M.; Caldwell, J. W.; Kollman, P. A.; Case, D. A., Development and testing of a general amber force field. J. Comput. Chem. 2004, 25, 1157-1173.

62. Jorgensen, W. L.; Chandrasekhar, J;; Madura, J. D.; Impey, R. W.; Klein, M. L., Comparison of Simple Potential Functions for Simulating Liquid Water. J. Chem. Phys. 1983, 79, 926-935.

63. Price, D. J.; Brooks III, C. L., A Modified TIP3P Water Potential for Simulation with Ewald Summation. J. Chem. Phys. 2004, 121, 10096-10103.

64. Martínez, L.; Andrade, R.; Birgin, E. G.; Martínez, J. M., PACKMOL: a package for building initial configurations for molecular dynamics simulations. J. Comput. Chem. 2009, 30, 2157-2164.

65. Huai, Z.; Shen, Z.; Sun, Z., Binding Thermodynamics and Interaction Patterns of Inhibitor-Major Urinary Protein-I Binding from Extensive Free-Energy Calculations: Benchmarking AMBER Force Fields. J. Chem. Inf. Model. 2021, 61, 284297.

66. Wang, X.; Sun, Z., Understanding PIM-1 kinase inhibitor interactions with free energy simulation. Phys. Chem. Chem. Phys. 2019, 21, 7544-7558.

67. Fan, S.; Nedev, H.; Vijayan, R.; Iorga, B. I.; Beckstein, O., Precise force-field-based calculations of octanol-water partition coefficients for the SAMPL7 molecules. J. Comput.-Aided Mol. Des. 2021, 35, 853-870.

68. Sun, Z. X.; Wang, X. H.; Zhang, J. Z. H., BAR-based Optimum Adaptive Sampling Regime for Variance Minimization in Alchemical Transformation. Phys. Chem. Chem. Phys. 2017, 19, 15005-15020.

69. Wang, X.; Tu, X.; Zhang, J. Z. H.; Sun, Z., BAR-based Optimum Adaptive Sampling Regime for Variance Minimization in Alchemical Transformation: The Nonequilibrium Stratification. Phys. Chem. Chem. Phys. 2018, 20, $2009-2021$.

70. Huai, Z.; Yang, H.; Li, X.; Sun, Z., SAMPL7 TrimerTrip host-guest binding affinities from extensive alchemical and endpoint free energy calculations. J. Comput.-Aided Mol. Des. 2021, 35, 117-129.

71. Procacci, P.; Cardelli, C., Fast Switching Alchemical Transformations in Molecular Dynamics Simulations. J. Chem. Theory Comput. 2014, 10, 2813-2823.

72. Macchiagodena, M.; Karrenbrock, M.; Pagliai, M.; Procacci, P., Virtual Double-System Single-Box for Absolute Dissociation Free Energy Calculations in GROMACS. J. Chem. Inf. Model. 2021.

73. Evans, D. J.; Searles, D. J., Equilibrium microstates which generate second law violating steady states. Phys. Rev. E 1994, 50, 1645-1648.

74. Evans, D. J.; Searles, D. J., The Fluctuation Theorem. Adv. Phys. 2002, 51, 1529-1585.

75. Crooks, G. E., Nonequilibrium Measurements of Free Energy Differences for Microscopically Reversible Markovian Systems. J. Stat. Phys. 1998, 90, 1481-1487.

76. Hummer, G., Fast-growth thermodynamic integration: Error and efficiency analysis. J. Chem. Phys. 2001, 114, 7330.

77. Abraham, M. J.; Murtola, T.; Schulz, R.; Páll, S.; Smith, J. C.; Hess, B.; Lindahl, E., GROMACS: High performance molecular simulations through multi-level parallelism from laptops to supercomputers. SoftwareX 2015, 1, 19-25.

78. Bussi, G.; Donadio, D.; Parrinello, M., Canonical sampling through velocity rescaling. J. Chem. Phys. $2007,126,2384$.

79. Nosé, S.; Klein, M. L., Constant pressure molecular dynamics for molecular systems. Molecular Physics 1983, 50, 1055-1076.

80. Parrinello, M.; Rahman, A., Polymorphic transitions in single crystals: A new molecular dynamics method. Journal of Applied Physics 1981, 52, 7182-7190.

81. Essmann, U.; Perera, L.; Berkowitz, M. L.; Darden, T.; Lee, H.; Pedersen, L. G., A smooth particle mesh Ewald method. J. Chem. Phys. 1995, 103, 8577-8593.

82. Sun, Z.; He, Q.; Zhihao, G.; Payam, K.; Huai, Z.; Liu, Z., A General Picture of Cucurbit[8]uril Host-Guest Binding: Recalibrating Bonded Interactions. 2021.

83. Morado, J.; Mortenson, P. N.; Verdonk, M. L.; Ward, R. A.; Essex, J. W.; Skylaris, C.-K., ParaMol: A Package for Automatic Parameterization of Molecular Mechanics Force Fields. J. Chem. Inf. Model. 2021, 61, $2026-2047$.

84. Wang, L.-P.; Chen, J.; Van Voorhis, T., Systematic parametrization of polarizable force fields from quantum chemistry data. J. Chem. Theory Comput. 2013, 9, 452-460.

85. Brandenburg, J. G.; Bannwarth, C.; Hansen, A.; Grimme, S., B97-3c: A revised low-cost variant of the B97-D density $33 / 70$ 
functional method. J. Chem. Phys. 2018, 148, 064104.

86. Neese, F., The ORCA program system. Wiley Interdisip. Rev. Comput. Mol. Sci. 2012, 2, 73-78.

87. Neese, F., Software update: the ORCA program system, version 4.0. Wiley Interdisip. Rev. Comput. Mol. Sci. 2018, 8, e1327.

88. MacFarlane, D. R.; Golding, J.; Forsyth, S.; Forsyth, M.; Deacon, G. B., Low viscosity ionic liquids based on organic salts of the dicyanamide anion. Chem. Commun. 2001, 1430-1431.

89. Jacquemin, J.; Husson, P.; Padua, A. A. H.; Majer, V., Density and viscosity of several pure and water-saturated ionic liquids. Green Chemistry 2006, 8, 172-180.

90. Vercher, E.; Orchillés, A. V.; Miguel, P. J.; Martínez-Andreu, A., Volumetric and Ultrasonic Studies of 1-Ethyl-3methylimidazolium Trifluoromethanesulfonate Ionic Liquid with Methanol, Ethanol, 1-Propanol, and Water at Several Temperatures. Journal of Chemical \& Engineering Data 2007, 52, 1468-1482.

91. Seoane, R. G.; Corderí, S.; Gómez, E.; Calvar, N.; González, E. J.; Macedo, E. A.; Domínguez, Á., Temperature Dependence and Structural Influence on the Thermophysical Properties of Eleven Commercial Ionic Liquids. Industrial \& Engineering Chemistry Research 2012, 51, 2492-2504.

92. Jarzynski, C., A Nonequilibrium Equality for Free Energy Differences. Physical Review Letters 1997, 78, $2690-2693$.

93. Jarzynski, C., Equilibrium free-energy differences from nonequilibrium measurements: A master-equation approach. Phys. Rev. E 1997, 56, 5018-5035.

94. Paliwal, H.; Shirts, M. R., A benchmark test set for alchemical free energy transformations and its use to quantify error in common free energy methods. J. Chem. Theory Comput. 2011, 7, 4115-34.

95. Wood, R. H.; Muhlbauer, W. C. F.; Thompson, P. T., Systematic errors in free energy perturbation calculations due to a finite sample of configuration space: sample-size hysteresis. J. Phys. Chem. 1991, 95, 6670-6675.

96. Gore, J.; Ritort, F.; Bustamante, C., Bias and error in estimates of equilibrium free-energy differences from nonequilibrium measurements. Proc. Natl. Acad. Sci. USA 2003, 100, 12564-12569.

97. Wang, X.; Sun, Z., A Theoretical Interpretation of Variance-based Convergence Citeria in Perturbation-based Theories. arXiv preprint arXiv:1803.03123 2018.

98. Kirkwood, J. G., Statistical Mechanics of Fluid Mixtures. J. Chem. Phys. 1935, 3, 300.

99. Pearlman, D. A.; Kollman, P. A., The lag between the Hamiltonian and the system configuration in free energy perturbation calculations. Journal of Chemical Physics 1989, 91, 7831-7839.

100. Straatsma, T. P.; Mccammon, J. A., Treatment of rotational isomers in free energy calculations. II. Molecular dynamics simulation study of 18-crown-6 in aqueous solution as an example of systems with large numbers of rotational isomeric states. J. Chem. Phys. 1989, 91, 3631-3637.

101. Darden, T.; Pearlman, D.; Pedersen, L. G., Ionic charging free energies: Spherical versus periodic boundary conditions. J. Chem. Phys. 1998, 109, 10921-10935.

102. Rocklin, G. J.; Mobley, D. L.; Dill, K. A.; Hünenberger, P. H., Calculating the binding free energies of charged species based on explicit-solvent simulations employing lattice-sum methods: An accurate correction scheme for electrostatic finite-size effects. J. Chem. Phys. 2013, 139, 184103.

103. Hub, J. S.; de Groot, B. L.; Grubmu“ller, H.; Groenhof, G., Quantifying artifacts in Ewald simulations of inhomogeneous systems with a net charge. J. Chem. Theory Comput. 2014, 10, 381-390.

104. Sun, Z.; Wang, X.; Song, J., Extensive Assessment of Various Computational Methods for Aspartate's pKa Shift. J. Chem. Inf. Model. 2017, 57, 1621-1639. 
<smiles></smiles>

EMIM

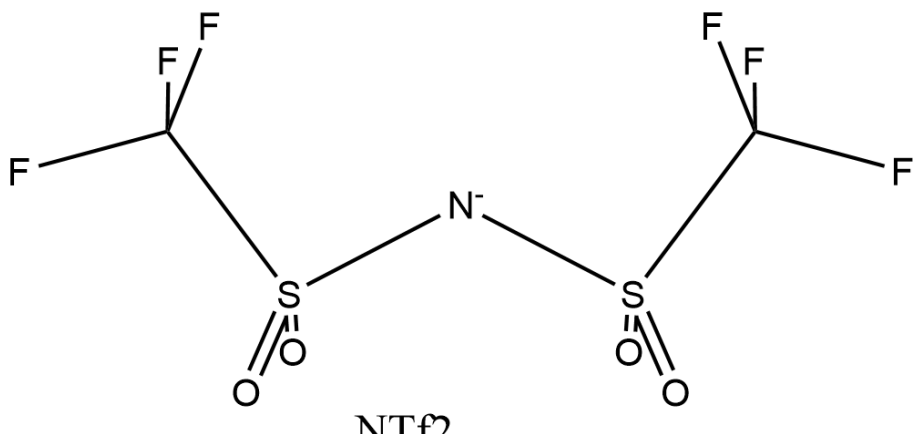

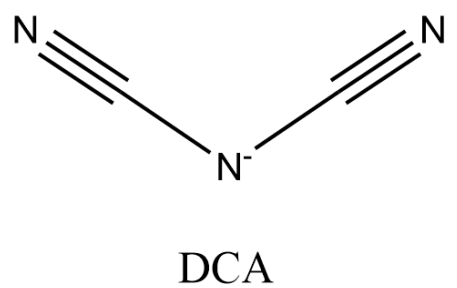

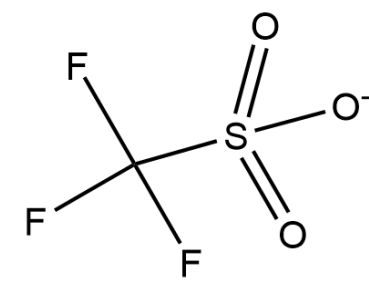

OTf

Fig. 1. The ionic species forming the ionic solvents considered in this work. The only cationic [EMIM] is combined with three different anionic species including [DCA], [NTf2] and [OTf], which gives three ionic liquids. The solute molecules under consideration are given in Table S1. 

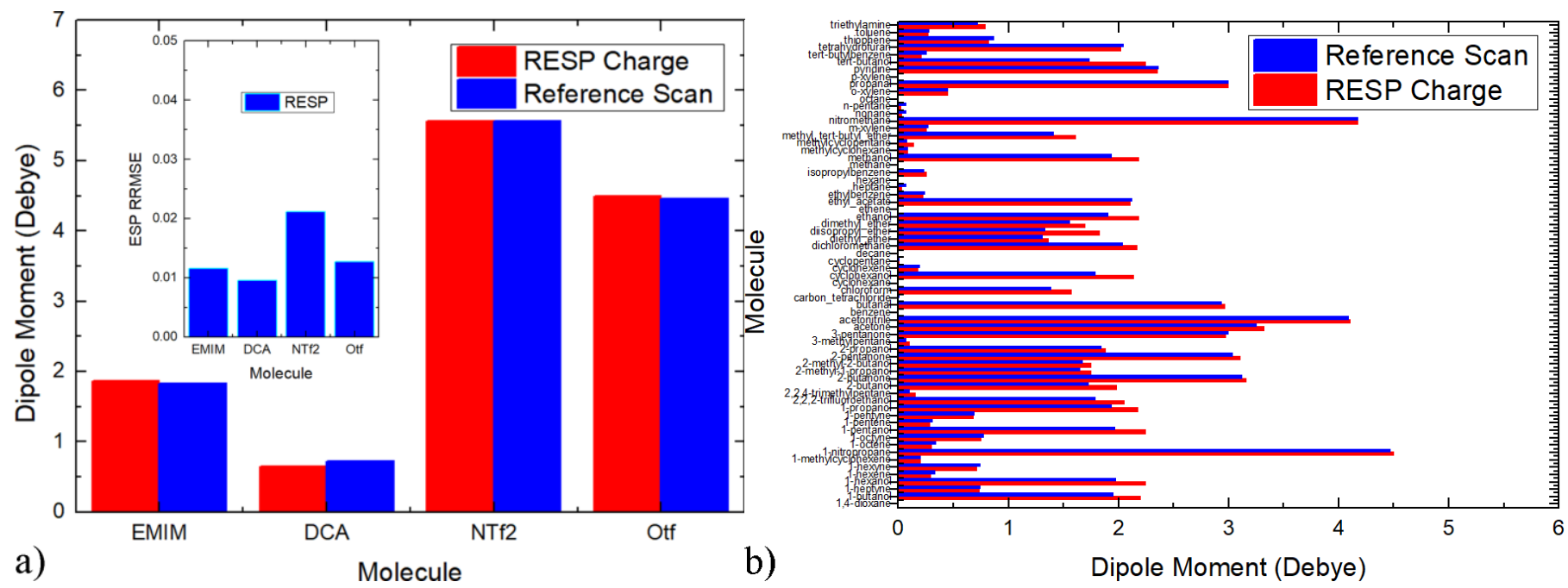

Fig. 2. Quality assessment for the RESP charge scheme. a) For the ions that form ionic liquids, the dipole moments produced by the fitted atomic charges and the reference HF/6-31G* scan, with the ESP relative error embedded in the inset. b) The dipole moments produced by the RESP charges and electronic structure calculations (i.e., HF/6-31G*) for all solutes under consideration. We can see that the regularized fitting produces charges that accurately reproduce the molecular ESP and the dipole moment at the target level for all molecules under consideration. 


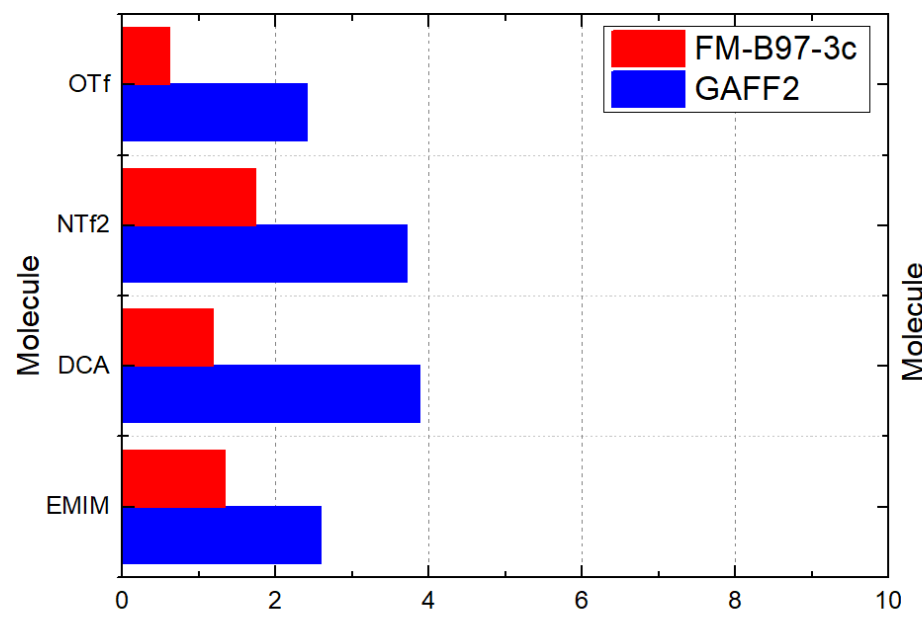

a)

RMSE Energy $(\mathrm{kcal} / \mathrm{mol})$

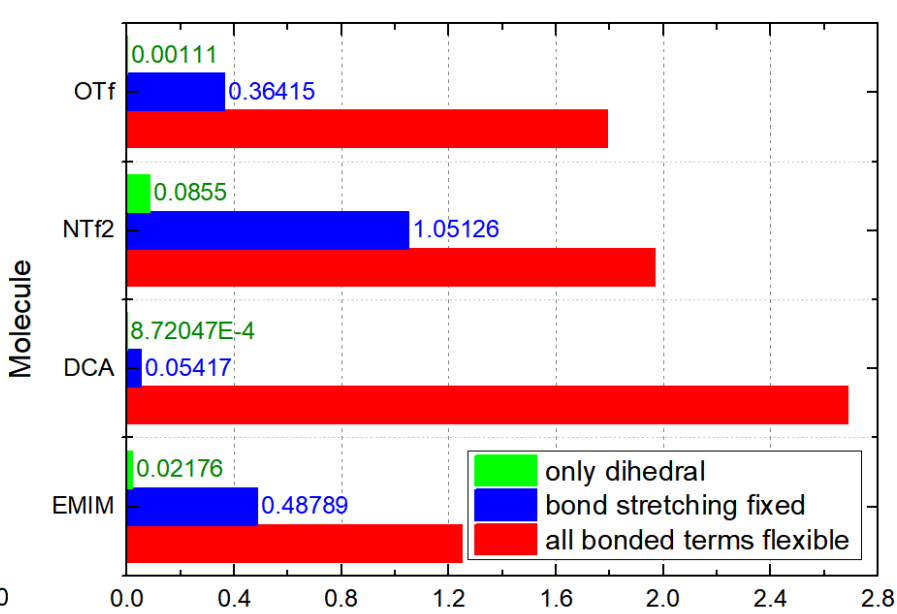

b)

Fig. 3. a) The errors/deviations (RMSEs) of the energetics produced by the initial guess GAFF2 and the refitted force field where all bonded terms are under adjustment. b) The decrease of the RMSEs of the system energy when shifting from the initial guess GAFF2 to the refitted force fields with three refitting procedures. The first one includes all bonded terms (i.e., bond stretching, angle bending and dihedral flipping) in the regularized parameter adjustment, the absolute RMSEs of which are presented in the first subplot. By contrast, in the second one we fix the bond stretching term and only refit angle bending and dihedral/torsional terms in order to separate the improvements of the error estimates due to the bond stretching term. Finally, in the third fitting procedure both bond stretching and angle bending terms are fixed to the original GAFF2 parameters and only torsional parameters are under refitting. Note that all force-field refitting procedures start from the pre-fitted GAFF2 parameter set. The configurations used in the calculation of error estimates in the all-terms, bond-stretching-fixed and dihedral-only refitting are generated independently. Thus, the error estimates in the three fitting procedures are independent. The first subplot clearly shows that significant improvements could be obtained upon GAFF2 refitting. However, when we only adjust the angle and dihedral parameters, the deviations of the force-field results from the ab initio target are not improved significantly. Further, when only dihedral terms are under refitting, the merit of refitting is negligible. These observations suggest that torsional/dihedral terms defined in the initial guess GAFF2 are not really problematic, while the other stiff degrees of freedom (i.e., bond stretching and angle bending) show relatively large errors and are the main source of improvements observed in the all-bondedterms-flexible refitting. 


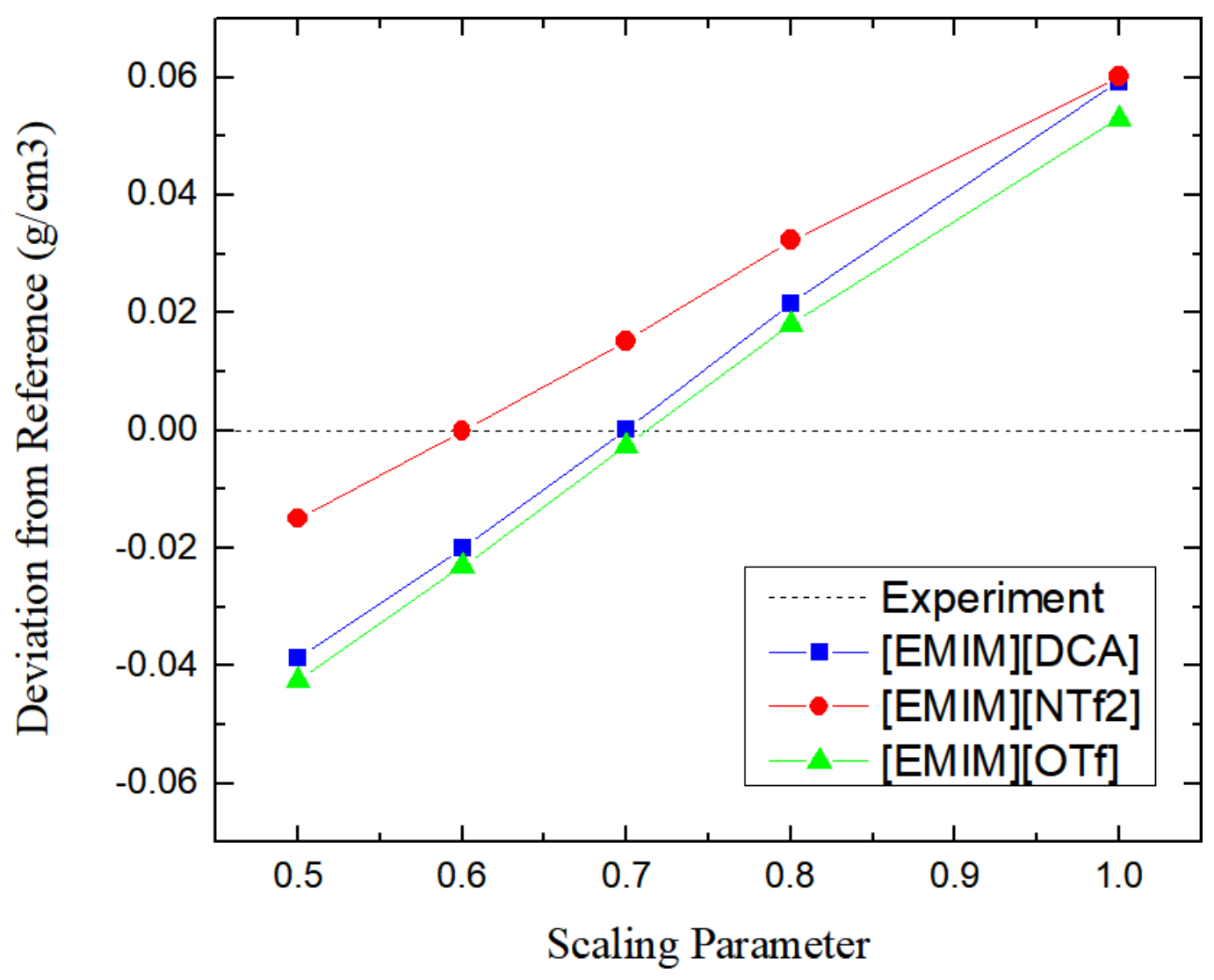

Fig. 4. The deviation of the simulated density from the experimental reference as a function of the scaling parameter for the atomic charges. The statistical uncertainty of the simulated density is quite small and thus is not shown. The experimental densities of the three ionic liquids under consideration (i.e., [EMIM][DCA], [EMIM][NTf2], and [EMIM][OTf]) are $1.06 \mathrm{~g} / \mathrm{cm}^{3}, 1.518 \mathrm{~g} / \mathrm{cm}^{3}$, and $1.384 \mathrm{~g} / \mathrm{cm}^{3}$, respectively. We can see that the optimal scaling factors for all systems lie somewhere between 0.6 and 0.7 , but the exact value shows system-dependence. 

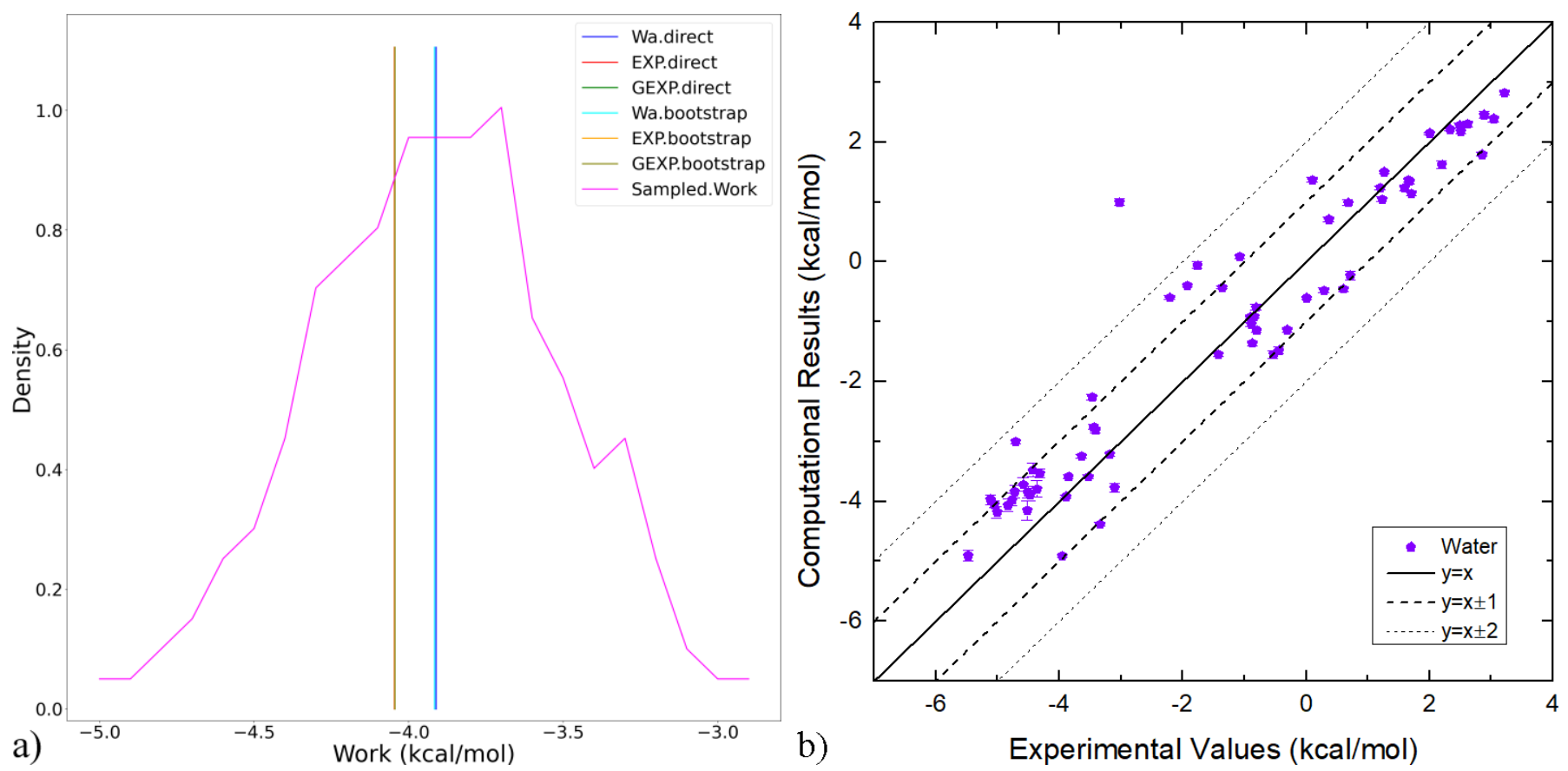

Fig. 5. a) Slow-growth, fast-growth and Gaussian approximated free energy estimates of hydration free energies calculated with directly sampled and bootstrap resampled work distributions superposed on the microscopic work distribution obtained from nonequilibrium alchemical transformations for 1,4-dioxane, b) the correlation between the calculated solvation free energies in water extracted from the EXP estimator and the experimental reference for all solutes under consideration. The exact values of these free energy estimates, the experimental references and the quality assessment metrics for these calculations are provided in Table S2. 

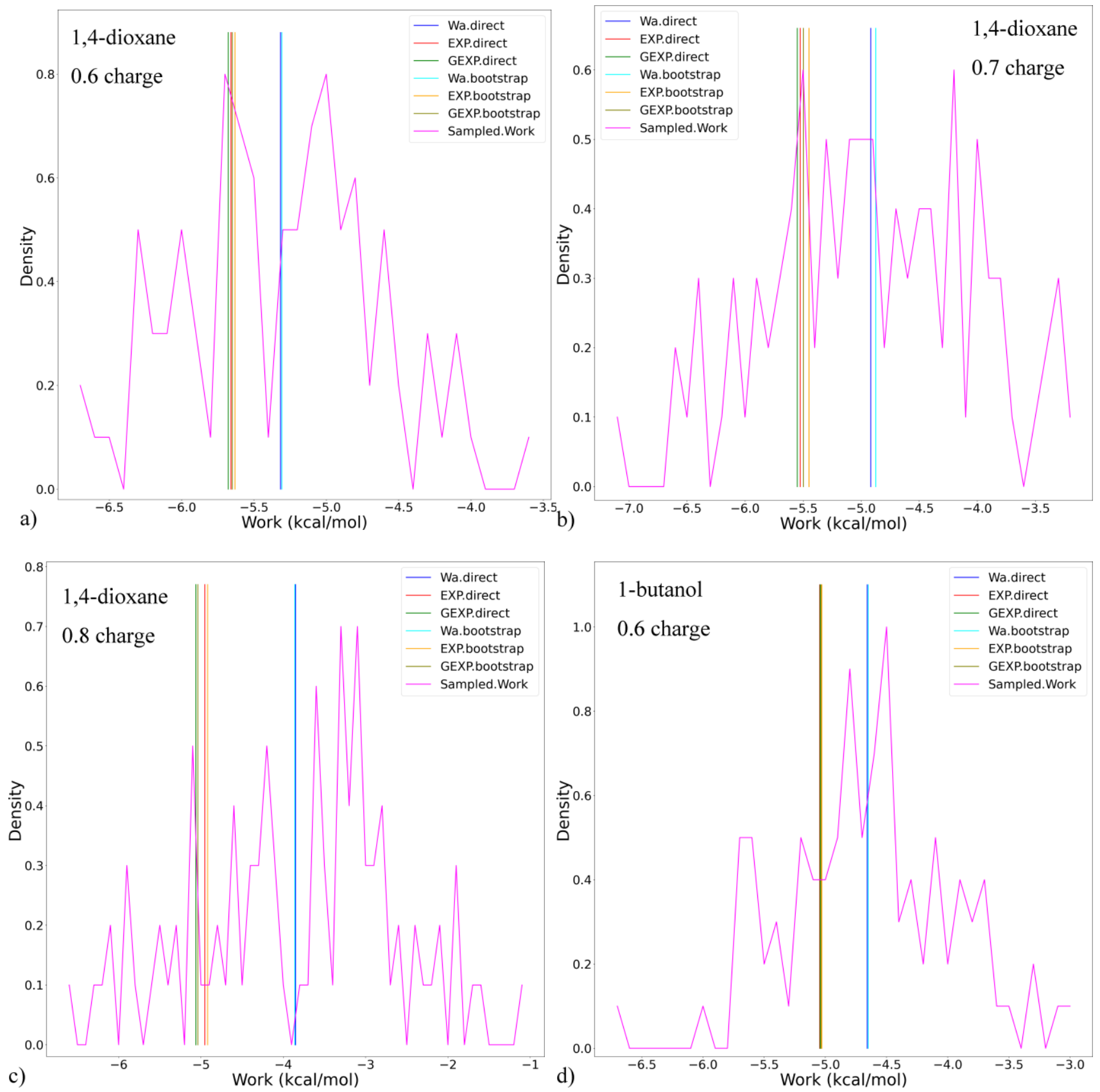

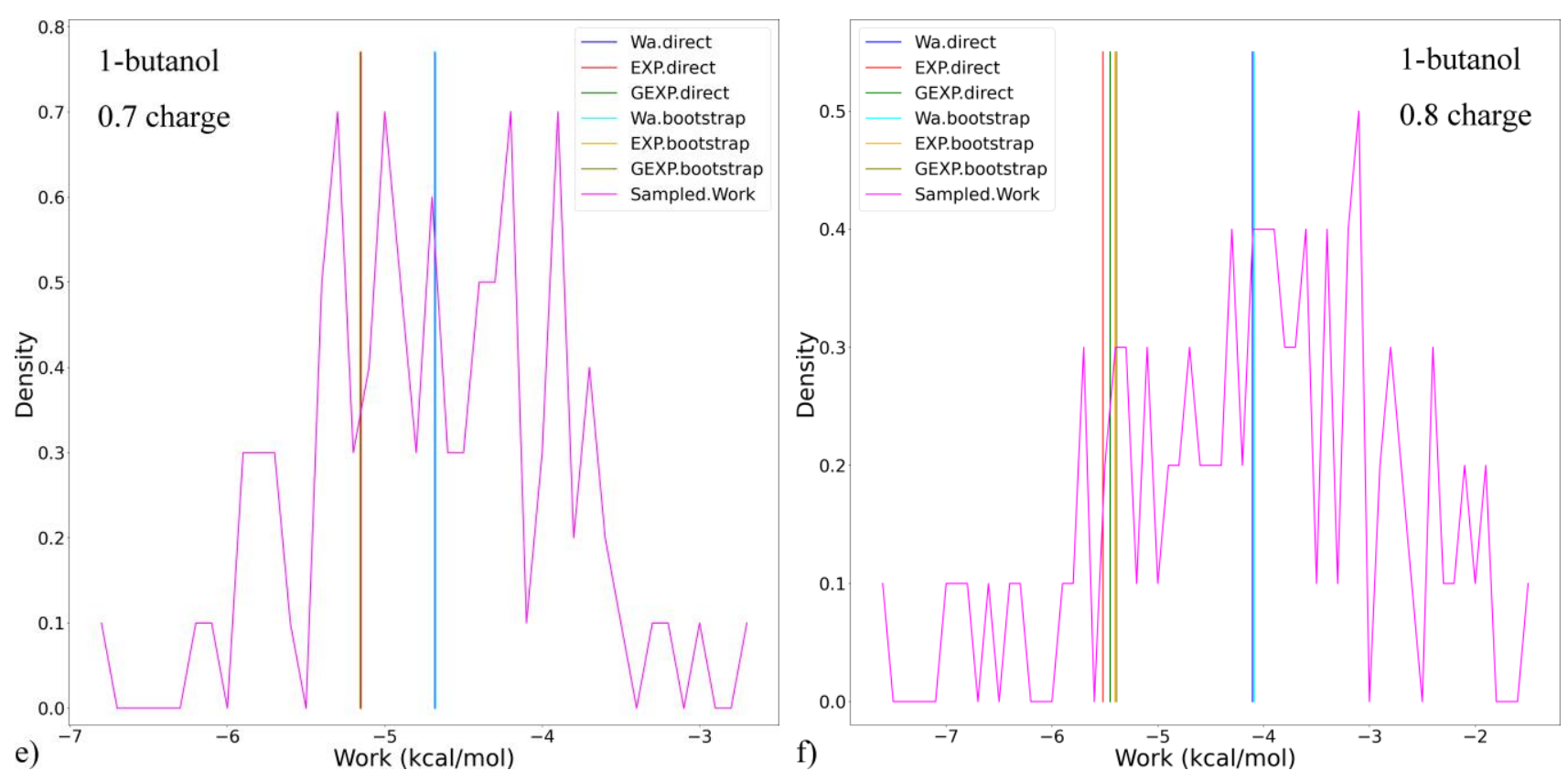

Fig. 6. Slow-growth, fast-growth and Gaussian approximated free energy estimates of solvation free energies calculated with directly sampled and bootstrap resampled work distributions superposed on the directly obtained microscopic work distribution from nonequilibrium alchemical transformations for a-c) 1,4-dioxane and d-f) 1-butanol in [EMIM][DCA] obtained with three charge scaling parameters $0.6,0.7$ and 0.8. Similar to the water solvent case, for all scaling parameters employed here and both solutes, the EXP and GEXP estimates agree quite well, which suggests near-equilibrium pulling and the resulting good convergence behavior of the nonequilibrium free energy simulation. The ordinary average of the microscopic works shows obvious differences from the other estimates, which indicates that the pulling is irreversibly conducted. The free energy estimates obtained with bootstrap resampling agree well with the direct results calculated from the sampled work distributions, which suggests that the hysteresis problem is negligible in the current pulling simulations. An interesting phenomenon about the free energy estimates under different charge-scaling factors is that for the first solute 1,4-dioxane, the solvation free energy becomes more negative when the atomic charges are scaled down, while for the second solute 1-butanol the opposite behavior is observed. This observation suggests that the monotonic response of the bulk properties to charge scaling may not be valid for solvation free energies, which complicates the current solvation benchmark. 


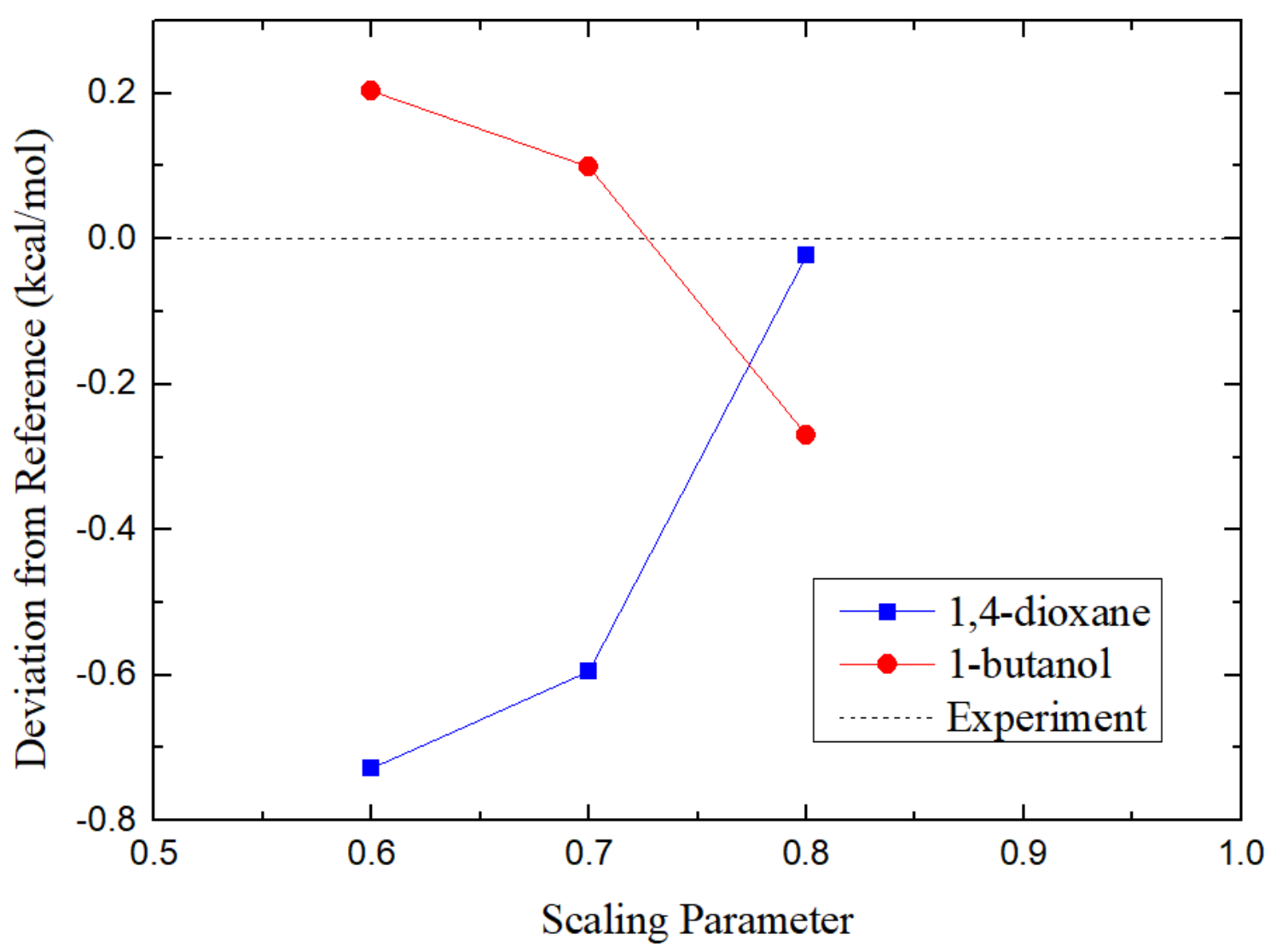

Fig. 7. The charge-scaling dependence of the deviation of the calculated solvation free energy from the experimental reference for the two solutes (1,4-dioxane and 1-butanol) in [EMIM][DCA]. The experimental solvation free energies of the two solutes in ionic liquids are $-4.93 \mathrm{kcal} / \mathrm{mol}$ and $-5.25 \mathrm{kcal} / \mathrm{mol}$. We can see that although both solutes exhibit some monotonic behavior with respect to the variation of the charge scaling parameter, one of them (1,4-dioxane) increases but the other (1-butanol) decreases monotonically. This opposite behavior suggests a solute-dependent response of the solvation free energy. As the whole set of solutes under consideration could show different monotonic behaviors, the overall solvation behavior is not expected to show some monotonic trend. If we only focus on the solvation of one molecule, the optimal charge scaling parameter would be 0.8 for 1,4-dioxane or 0.7 for 1-butanol, which also shows solutedependence. 


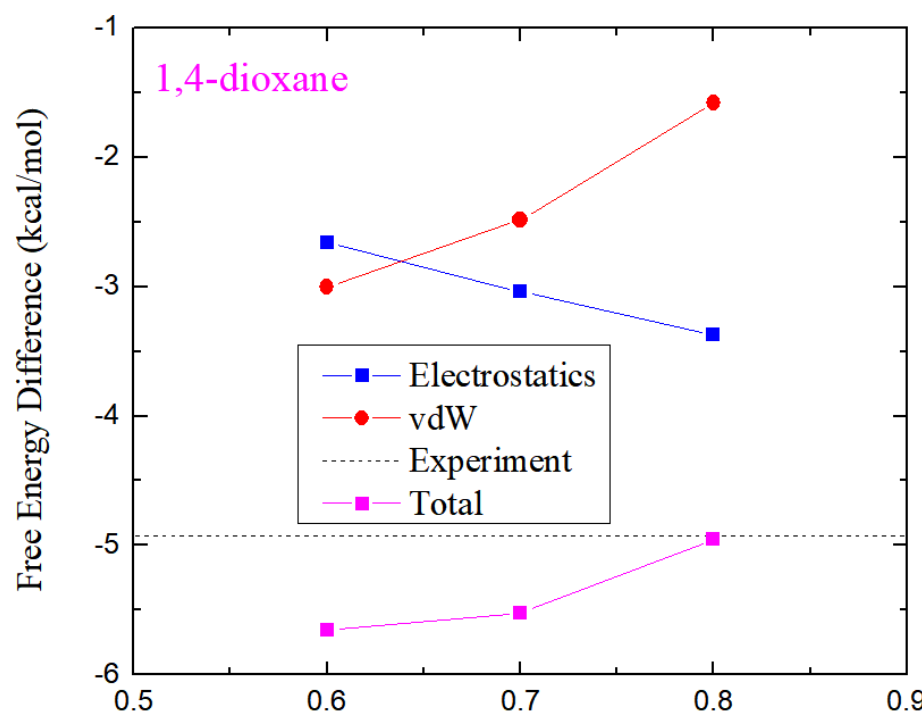

a)

Scaling Parameter

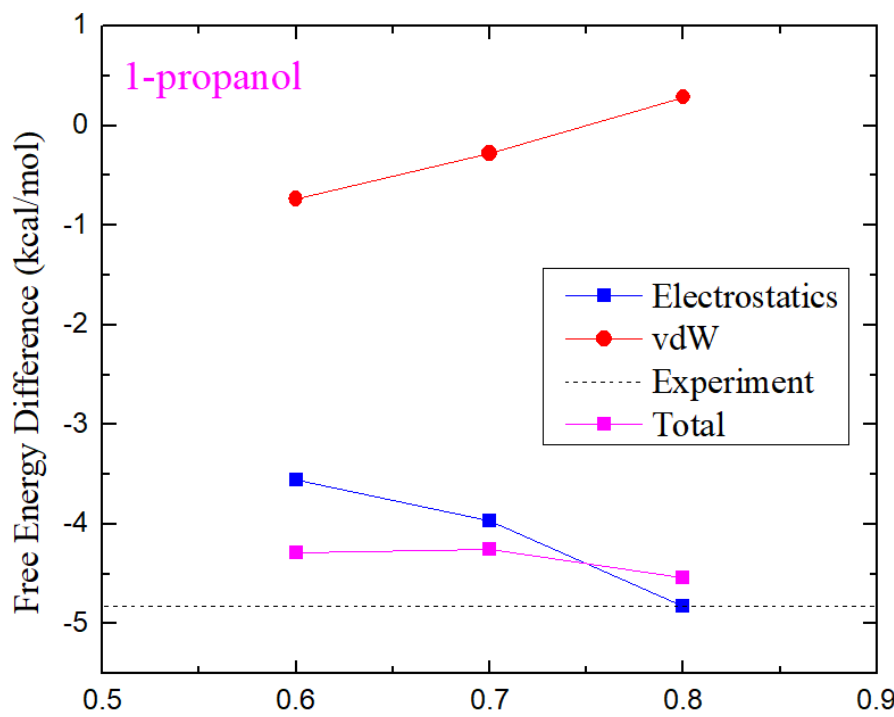

c)

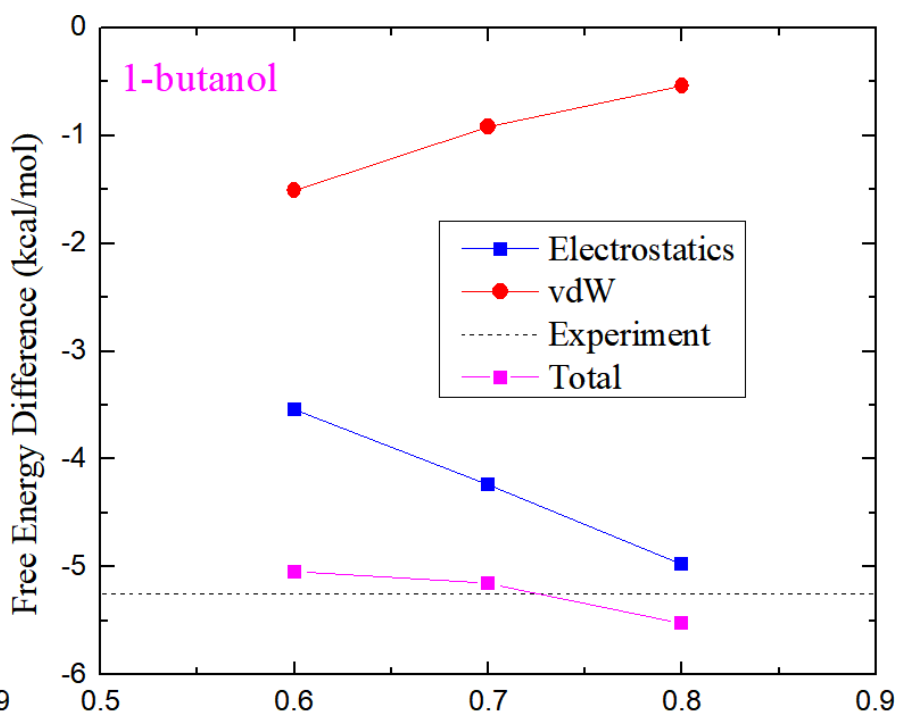

b)

\section{9}

Scaling Parameter

Fig. 8. The charge-scaling dependences of electrostatic and vdW components of the solvation free energy in $[\mathrm{EMIM}][\mathrm{DCA}]$ and their sum (i.e., total solvation free energy) as well as the experimental reference for a) 1,4-dioxane, b) 1-butanol and c) 1-propanol. It is clearly shown that the solvation free energy of 1,4-dioxnae increases monotonically (i.e., more positive) as the scaling parameter increases, while 1-butanol exhibits the opposite behavior. This solute-dependent behavior originates from the competing contributions from electrostatics and vdW interactions, which behaves differently when the scaling parameter is varied. The last example 1-propanol shows an intermediate behavior. Namely, its solvation free energy seems irrelevant to the scaling parameter, which arises from its similar electrostatic and vdW responses to the scaling-parameter variation. 

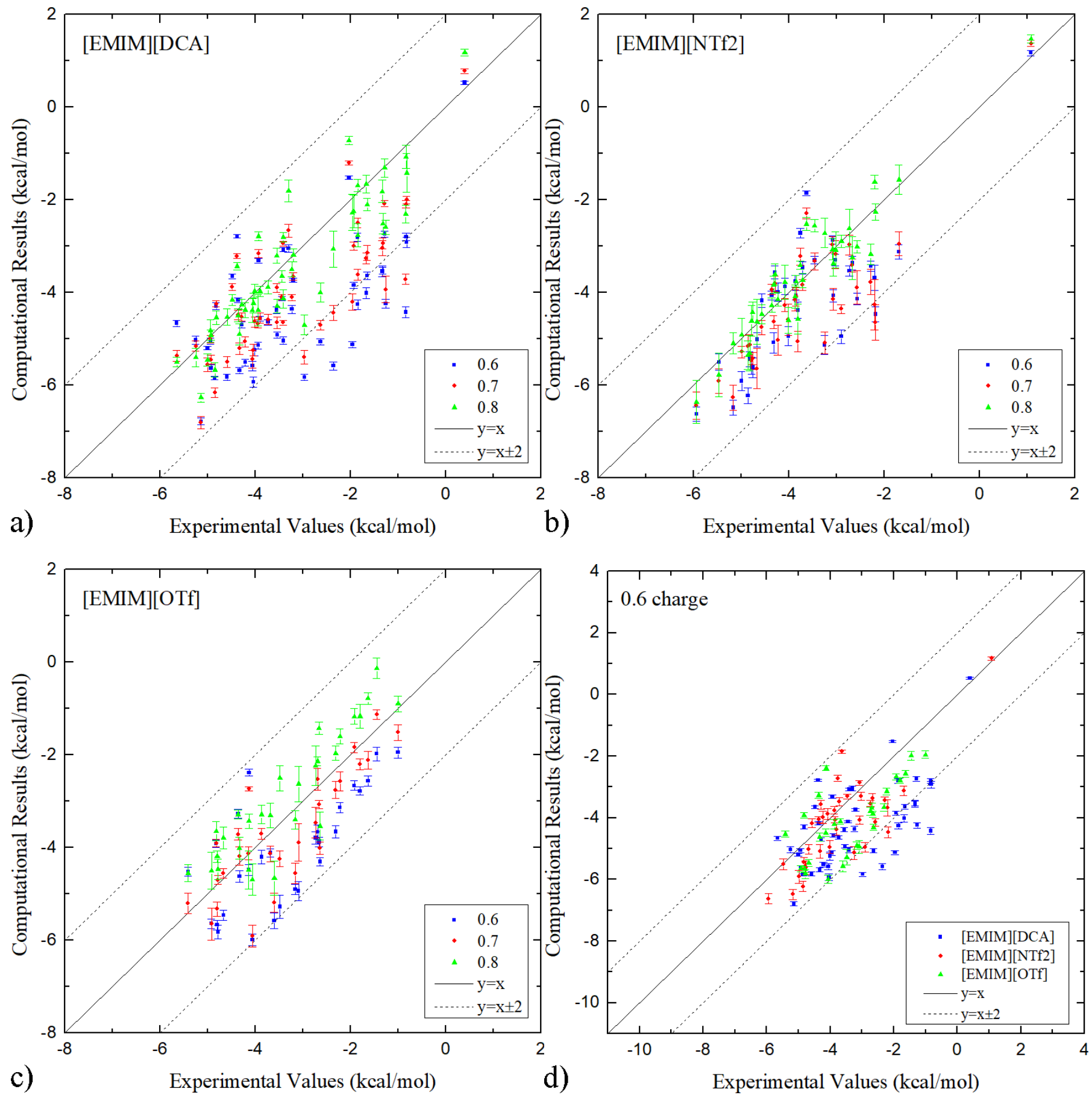


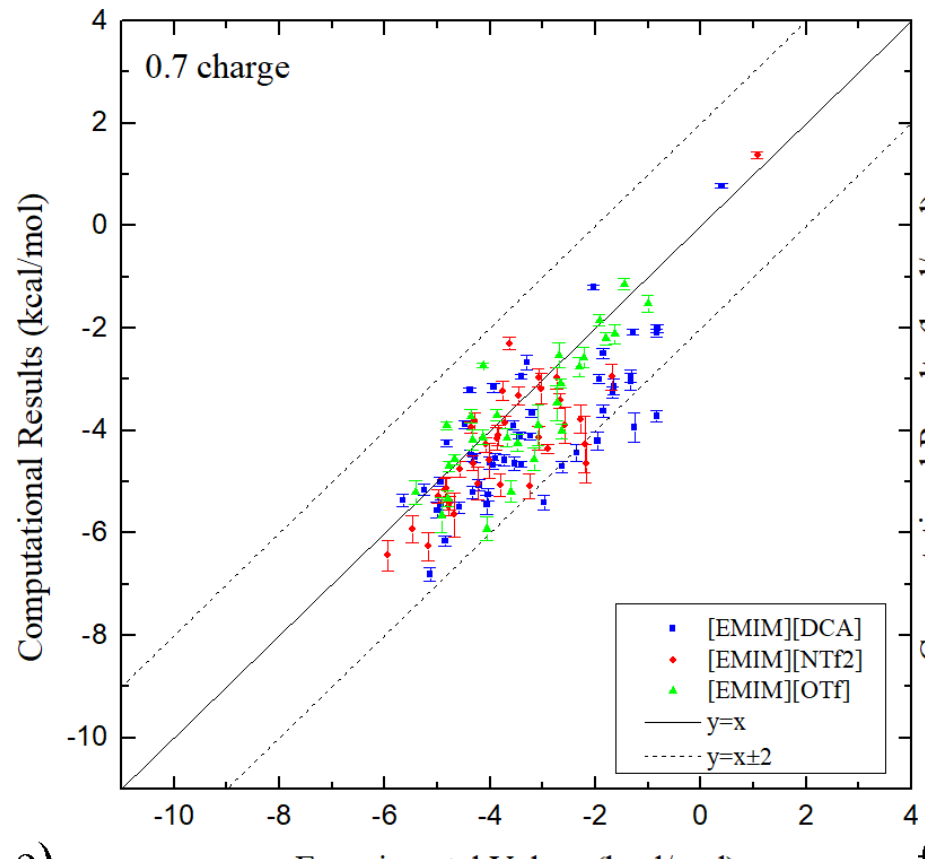

e)

Experimental Values (kcal/mol)

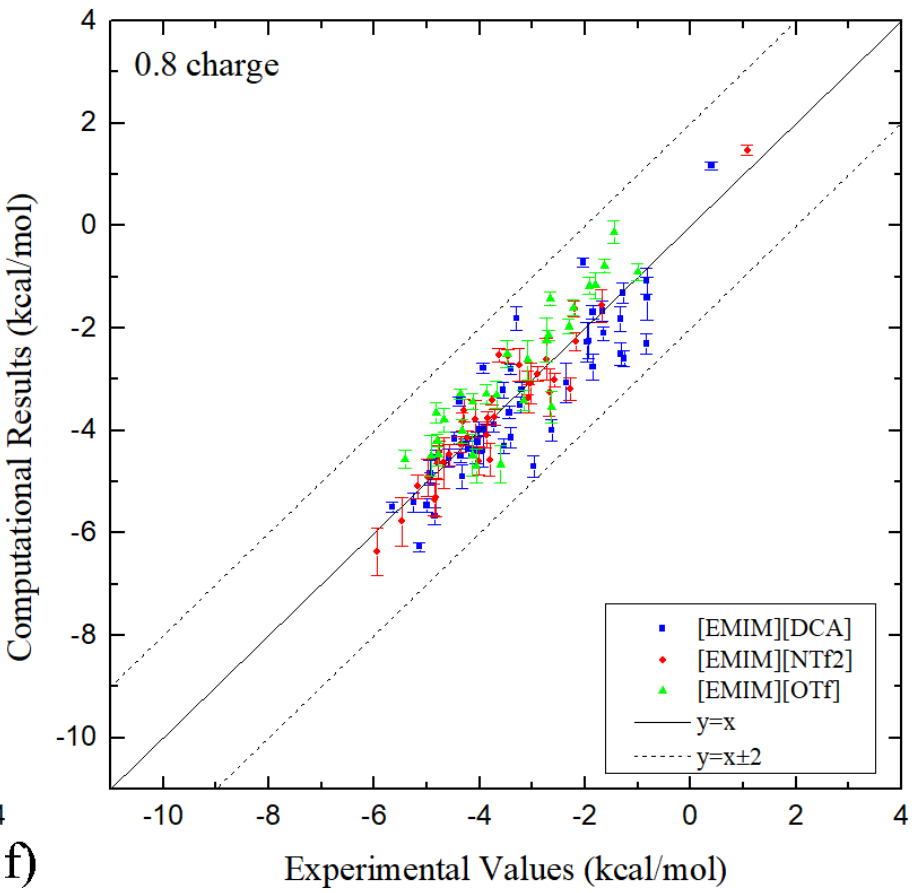

Fig. 9. The calculation-experiment correlation for EXP estimates of solvation free energies in a) [EMIM][DCA], b) [EMIM][NTf2] and c) [EMIM][OTf] under three scaling factors of 0.6, 0.7 and 0.8. And the experiment-calculation correlation for solvation free energies in all ionic liquids with the scaling factor of d) 0.6 , e) 0.7 , and f) 0.8 . The exact values of these solvation free energies, the experimental references and the quality assessment metrics are provided in Table S3-11. 


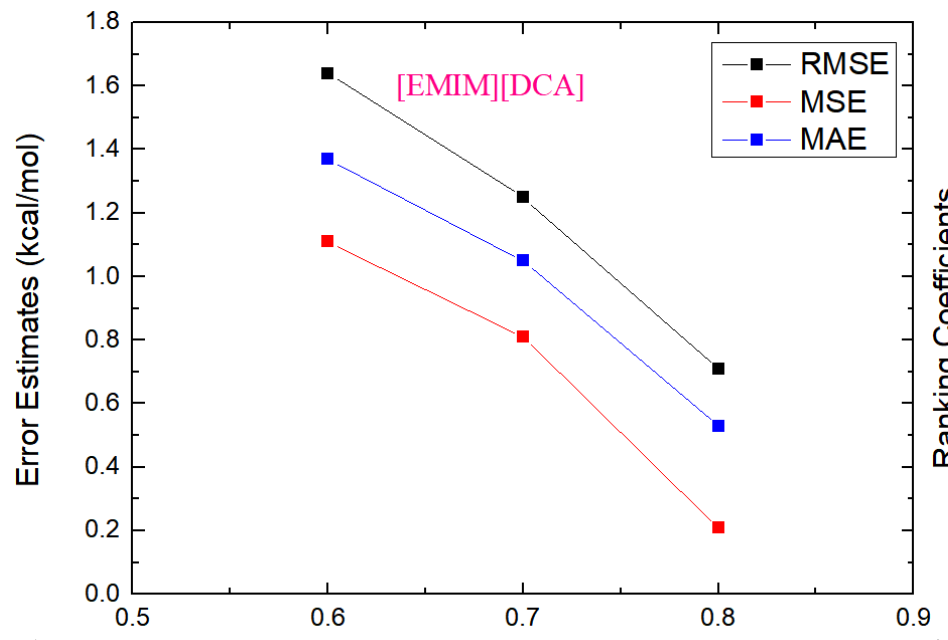

a)

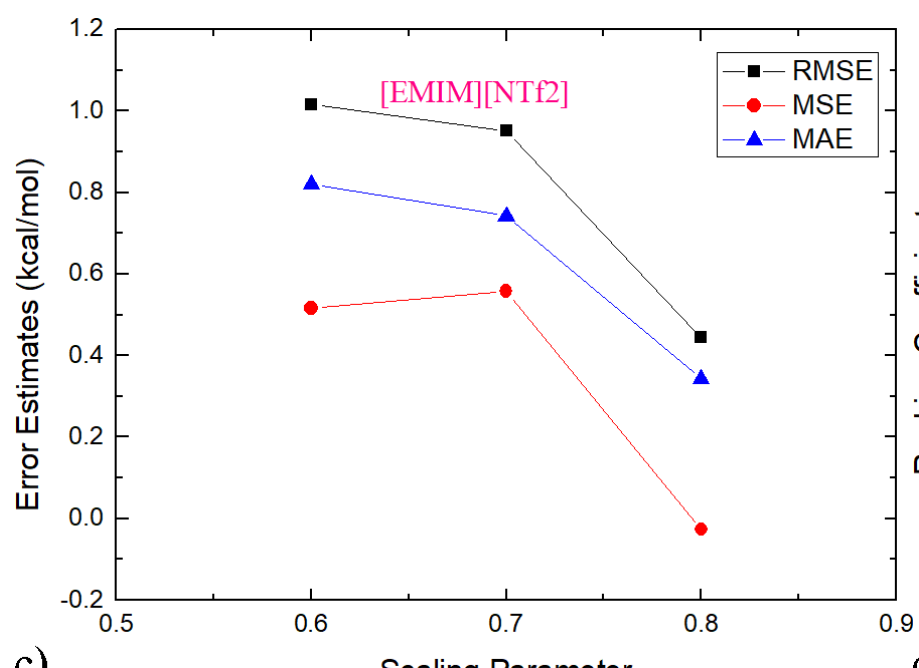

c)

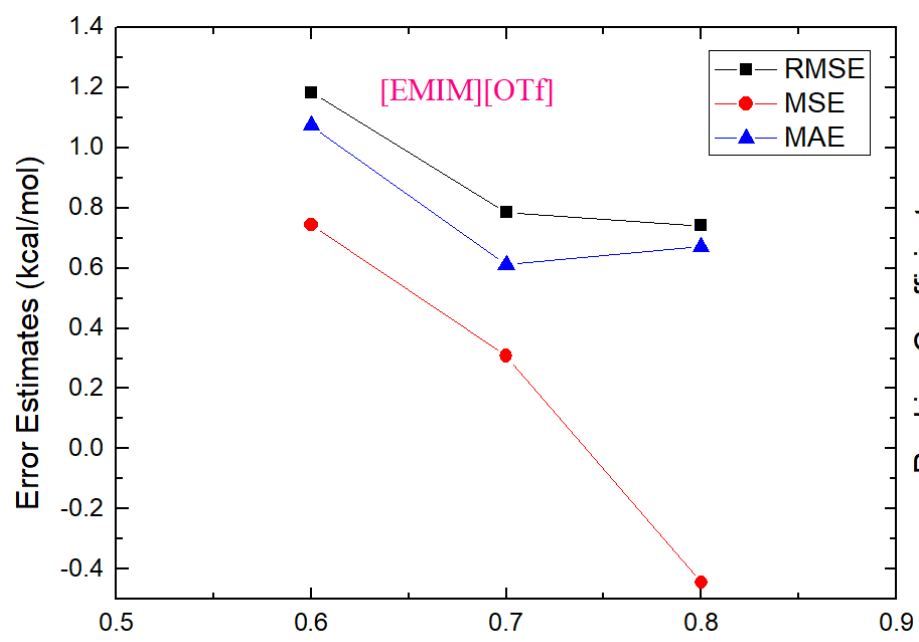

e)

Scaling Parameter

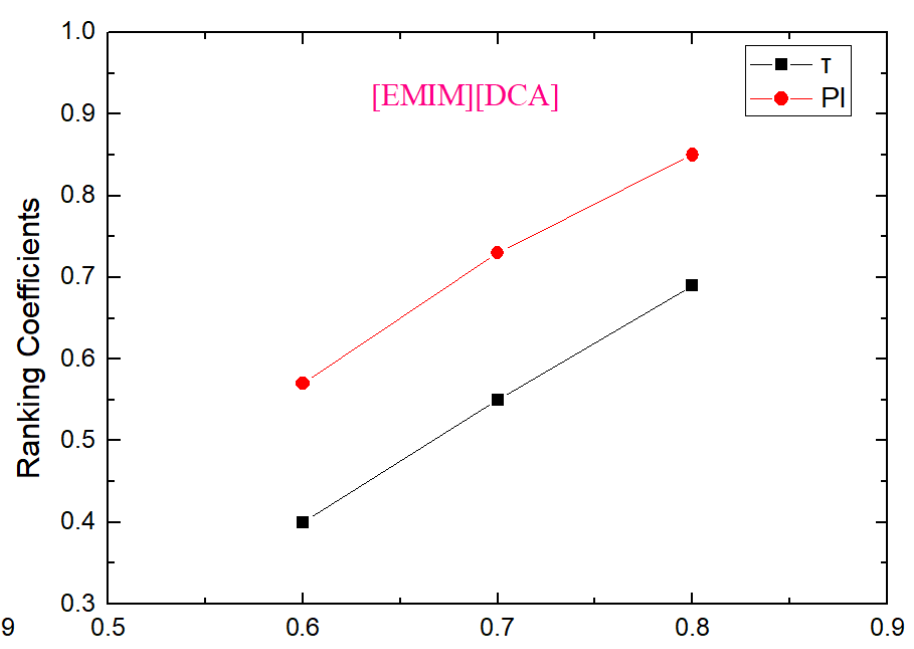

b)

Scaling Parameter

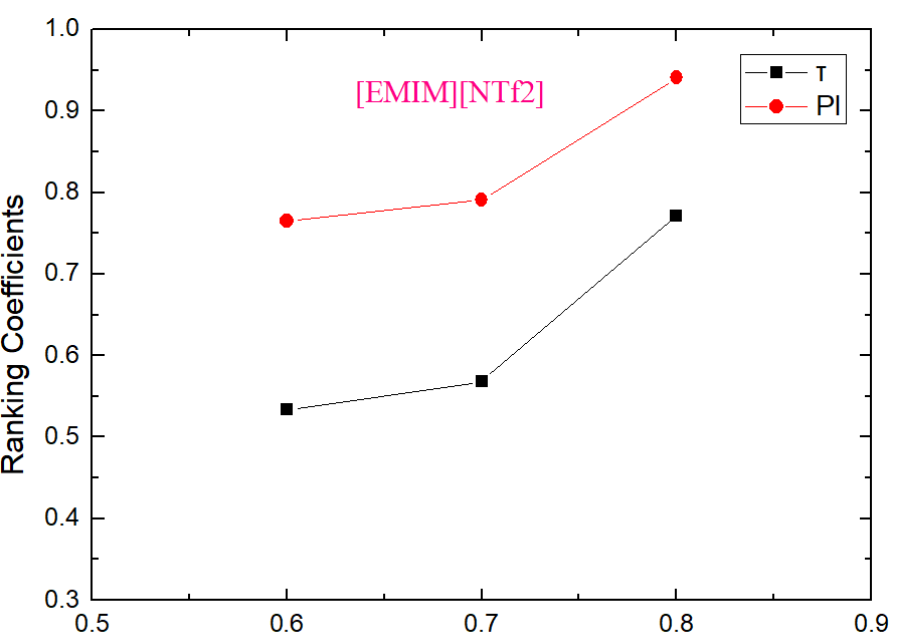

d)

Scaling Parameter

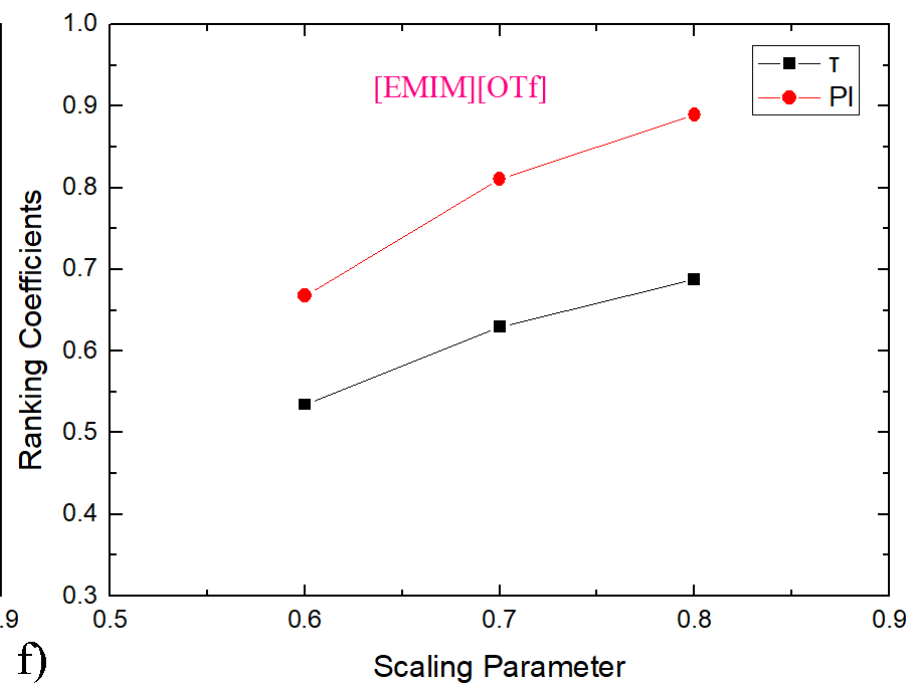




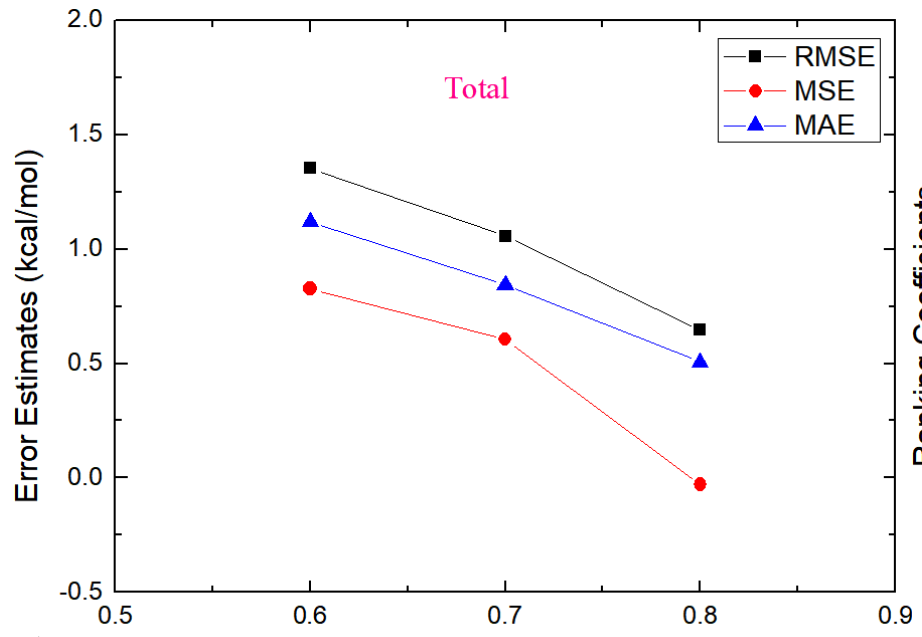

g)

Scaling Parameter

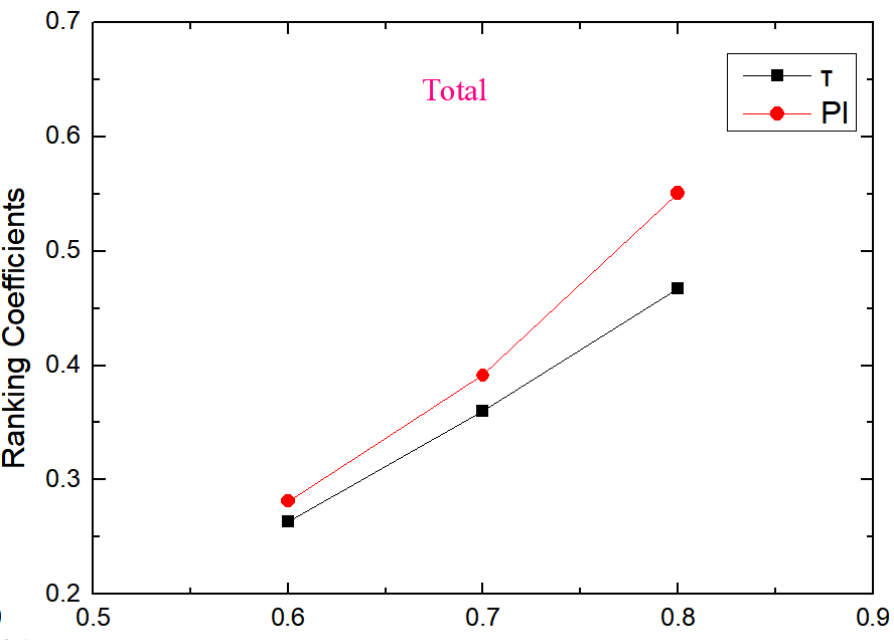

h)

Scaling Parameter

Fig. 10. Scaling-factor-dependence of quality metrics for infinite-dilution solvation free energies in ionic liquids: a-b) the ionic liquids [EMIM][DCA], c-d) [EMIM][NTf2], e-f) [EMIM][OTf] and g-h) all soluteionic-liquids pairs. The error metrics are shown in subplots a, c, e, and g, while in the other the ranking coefficients are presented. The best charge scaling parameter for the first ionic solvent [EMIM][DCA] seems to be 0.8 , although we cannot omit the possibility of better results under further enlarged scaling factors due to the monotonically decreasing behaviors of error metrics and monotonically increasing behaviors of ranking coefficients. However, as this value of 0.8 ensures highly accurate calculation of solvation thermodynamics and maintains a good level of density property of the bulk solvent simultaneously, this value is recommended in the modelling of this ion pair. The second ionic liquids [EMIM][NTf2] shows a similar behavior. Namely, its RMSE decreases and ranking coefficients increase monotonically when the scaling parameter increases, which gives the 0.8 solution to the charge scaling problem. This value is 0.2 larger than the density-derived estimate 0.6 . A worth noting observation for this solvation [EMIM][NTf2] set is that the accuracy of the calculated solvation free energies is already very good with the scaling parameter of 0.7 (i.e., RMSE $<1 \mathrm{kcal} / \mathrm{mol}$ ). Interestingly, for the third ionizing solvent [EMIM][OTf], the densityderived solution 0.7 is already good enough to produce satisfactory solvation thermodynamics that are in good agreement with the experimental references, and the RMSE values obtained with scaling factors 0.7 and 0.8 are very similar. However, the ranking coefficients still exhibit monotonic increasing behaviors when the scaling factor is increased. Combining error metrics and ranking coefficients, it can be concluded that for this ionic solvent the best scaling parameter is 0.8 , which is still 0.1 larger than the density-derived solution. In the last two subplots, the solvation free energies in all ionic liquids are combined to give the overall estimates of error metrics and ranking coefficients, which is used to investigate whether there is a universal scaling factor that produces satisfactory results of these solvation thermodynamics. Interestingly, it 
is indeed observed that this general solution exists. The error metrics and ranking coefficients show consistent monotonic behaviors with respect to the scaling factor, which leads to the best solution of 0.8 that is still at least 0.1 larger than the density-derived estimates. Therefore, we can conclude that for all ionic liquids under investigation, the solvation-free-energy-derived scaling factor is slightly higher than the density-derived one. 

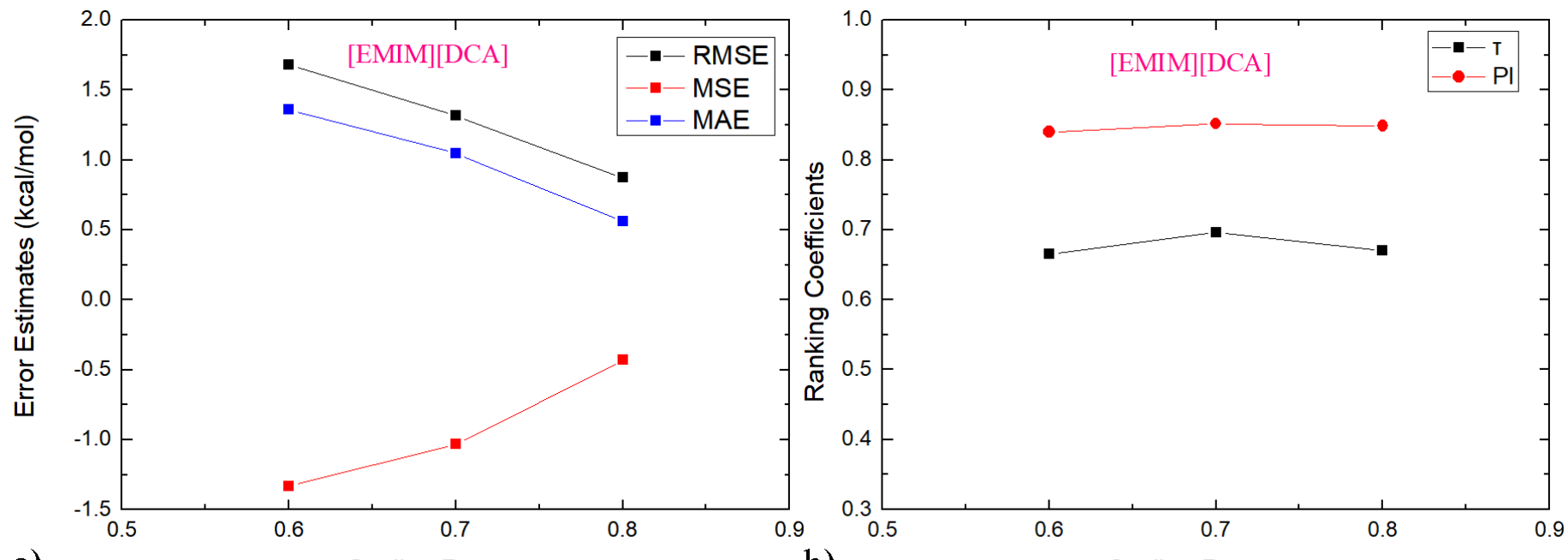

a)

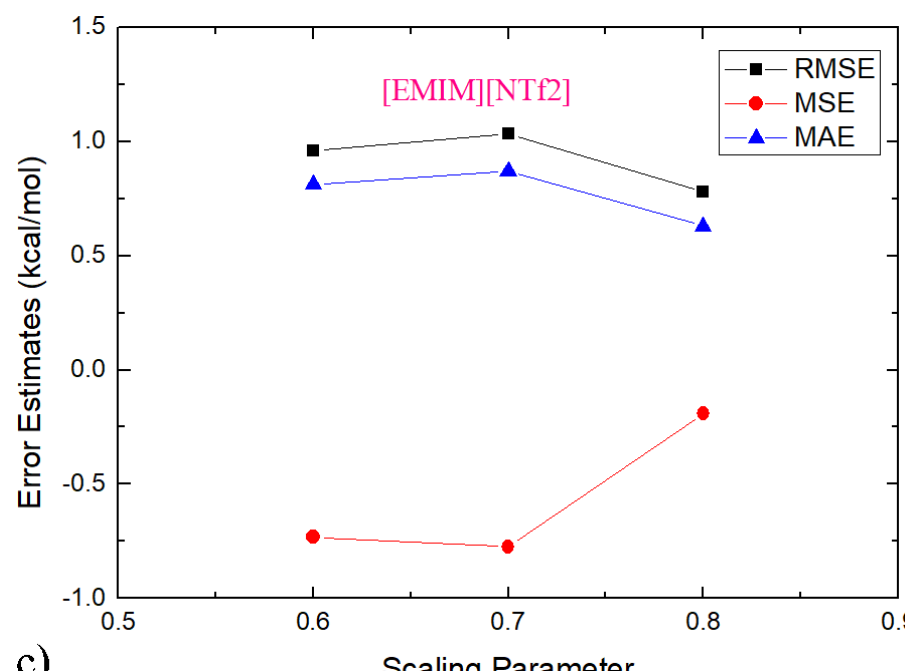

c)

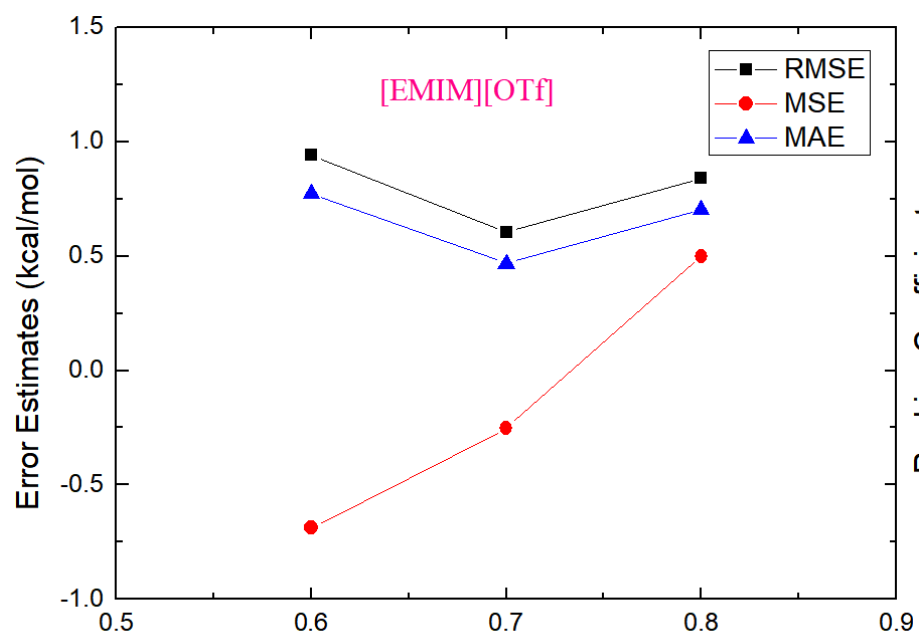

e)

Scaling Parameter b)

Scaling Parameter

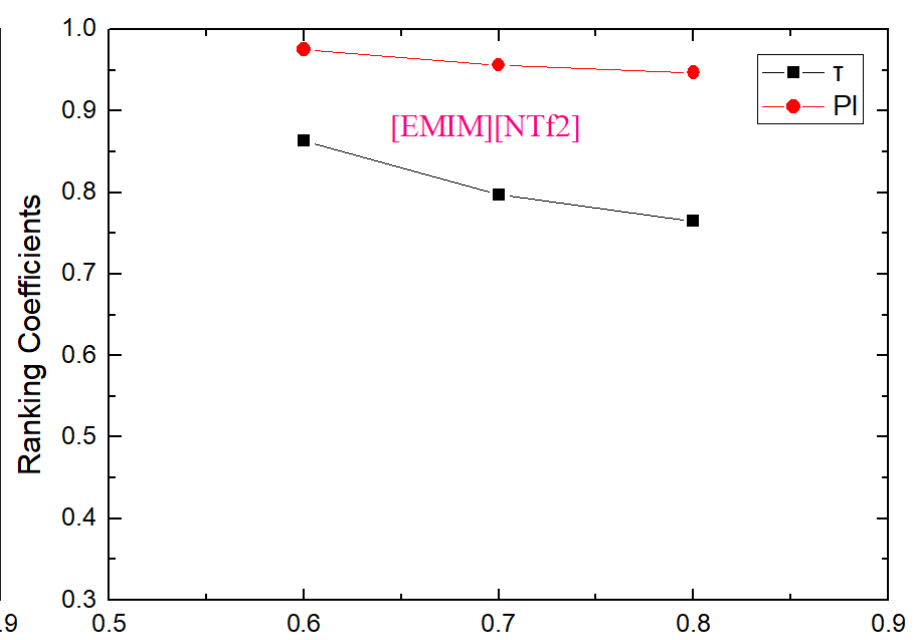

d)

Scaling Parameter

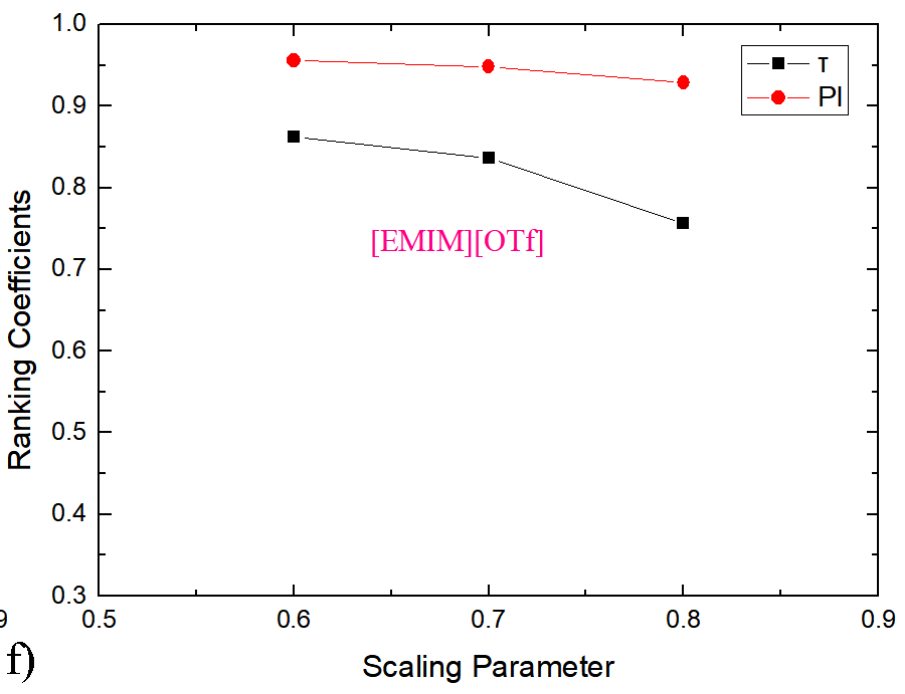



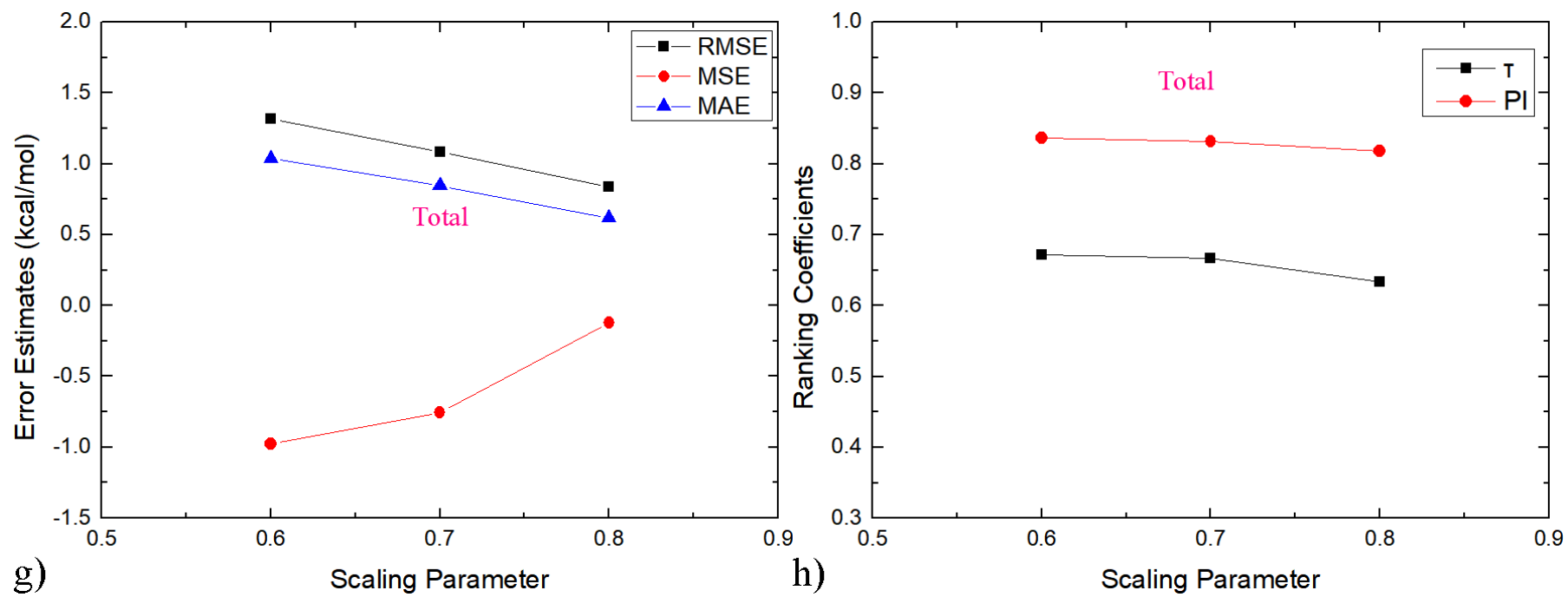

Fig. 11. Scaling-factor-dependence of quality metrics for the water-ionic-liquids transfer free energies: $a-b)$ the ionic liquids [EMIM][DCA], c-d) [EMIM][NTf2], e-f) [EMIM][OTf] and g-h) all solute-ionic-liquids pairs. The error metrics are shown in subplots a, c, e, and g, while in the other the ranking coefficients are presented. The EXP estimate is used in this benchmark. As the transfer free energy of a selected solute can be easily obtained by taking the difference between its hydration free energy and its solvation free energy in ionic liquids, the values of which are already provided in the tables shown in the supporting information, we do not summarize the exact values of these transfer free energies in a table. 


\title{
Supporting Information
}

\section{Molecular Modelling of Ionic Liquids: Force-Field Validation and \\ Thermodynamic Perspective from Large-Scale Fast-Growth Solvation Free Energy Calculations}

\author{
Zhaoxi Sun ${ }^{1 *}$, Mao Wang 2 , Qiaole He ${ }^{3}$, Zhirong Liu ${ }^{1}$ \\ ${ }^{I}$ Beijing National Laboratory for Molecular Sciences, College of Chemistry and Molecular Engineering, Peking \\ University, Beijing 100871, China \\ ${ }^{2}$ NCS Testing Technology Co., Ltd., No. 13, Gaoliangqiao Xiejie, Beijing 100081, China \\ ${ }^{3}$ AI Department of Enzymaster (Ningbo) Bio-Engineering Co., Ltd., North Century Avenue 333, 315100 Ningbo, \\ China
}

*To whom correspondence should be addressed: z.sun@pku.edu.cn 
Fig. S1. Time series of the deviation of the MM (GAFF2 and the refitted parameter set) energetics from the ab initio B97-3c reference during $10 \mathrm{~ns}$ trajectories generated at $300 \mathrm{~K}$ in vacuo for ions forming ionic liquids. The sampling interval is $10 \mathrm{ps}$ and there are 1000 independent configurations in total.
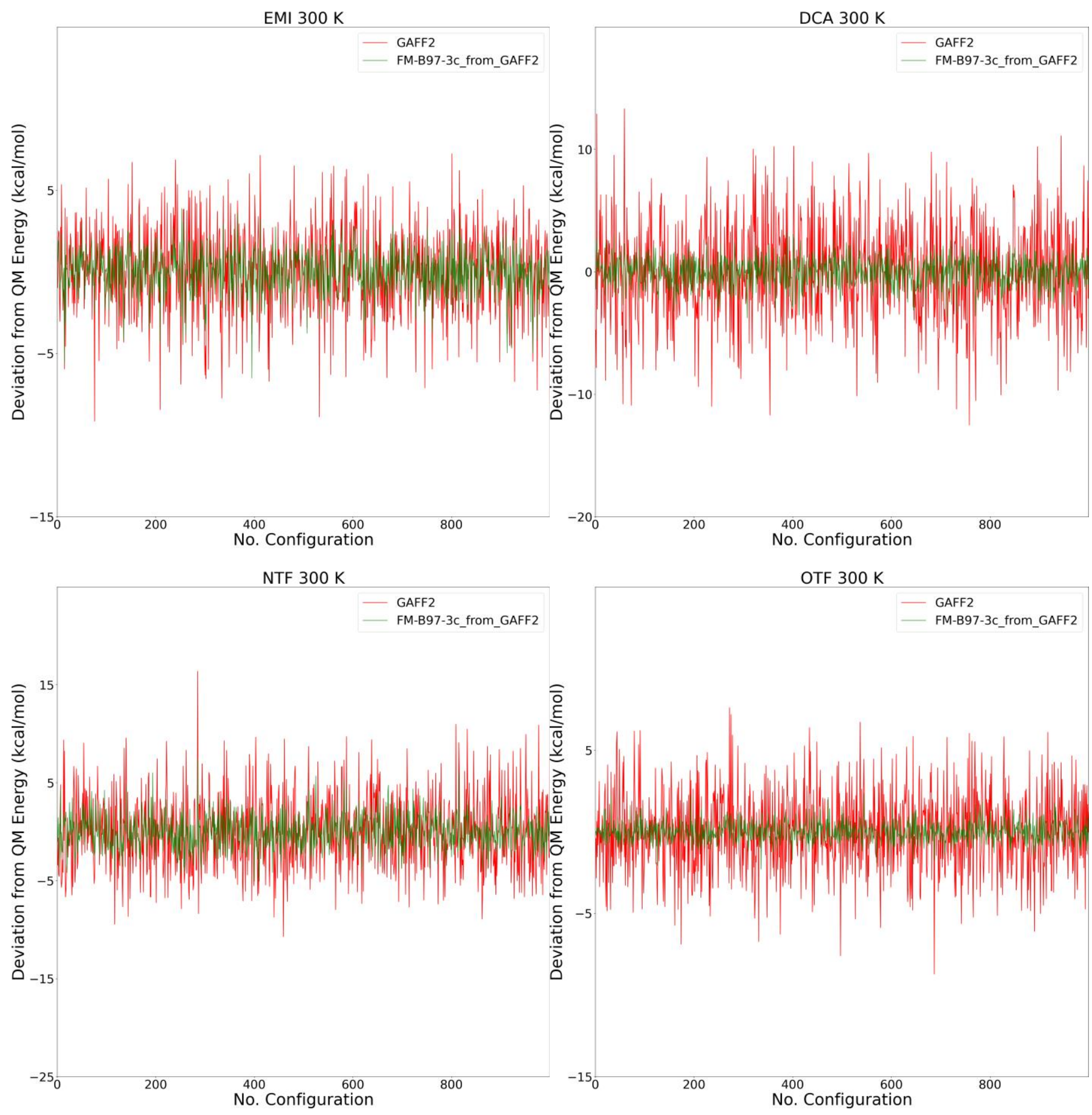
Table S1. Solutes considered in this work.

\begin{tabular}{ccc}
\hline 1,4-dioxane & 3-methylpentane & hexane \\
1-butanol & 3-pentanone & isopropylbenzene \\
1-heptyne & acetone & methane \\
1-hexanol & acetonitrile & methanol \\
1-hexene & benzene & methylcyclohexane \\
1-hexyne & butanal & methylcyclopentane \\
1-methylcyclohexene & carbon_tetrachloride & methyl_tert-butyl_ether \\
1-nitropropane & chloroform & m-xylene \\
1-octene & cyclohexane & nitromethane \\
1-octyne & cyclohexanol & nonane \\
1-pentanol & cyclohexene & n-pentane \\
1-pentene & cyclopentane & octane \\
1-pentyne & decane & o-xylene \\
1-propanol & dichloromethane & propanal \\
2,2,2-trifluoroethanol & diethyl_ether & p-xylene \\
2,2,4-trimethylpentane & diisopropyl_ether & pyridine \\
2-butanol & dimethyl_ether & tert-butanol \\
2-butanone & ethanol & tert-butylbenzene \\
2-methyl-1-propanol & ethene & tetrahydrofuran \\
2-methyl-2-butanol & ethyl_acetate & thiophene \\
2-pentanone & ethylbenzene & toluene \\
2-propanol & heptane & triethylamine \\
\hline
\end{tabular}


Table S2. Hydration free energy of all solutes under consideration in kcal/mol. $\Delta G_{W_{a}}$ is the slow-growth estimate, $\Delta G_{\mathrm{EXP}}$ represents exponential averaging, and $\Delta G_{\mathrm{GEXP}}$ is its Gaussian approximation. SD represents the standard error of the free energy estimate, which is obtained from numerical bootstrap resampling. MSE, MAE, RMSE, $\tau$, and PI serve as quality measurements.

\begin{tabular}{|c|c|c|c|c|c|c|c|}
\hline Solute & Experiment & $\Delta G_{W_{a}}$ & $\mathrm{SD}$ & $\Delta G_{\mathrm{EXP}}$ & $\mathrm{SD}$ & $\Delta G_{\mathrm{GEXP}}$ & $\mathrm{SD}$ \\
\hline 1,4-dioxane & -5.05 & -3.90 & 0.05 & -4.05 & 0.06 & -4.04 & 0.06 \\
\hline 1-butanol & -4.72 & -2.73 & 0.15 & -3.83 & 0.10 & -5.27 & 0.25 \\
\hline 1-heptyne & 0.61 & -0.31 & 0.04 & -0.45 & 0.04 & -0.46 & 0.04 \\
\hline 1-hexanol & -4.36 & -2.69 & 0.14 & -3.79 & 0.14 & -4.50 & 0.23 \\
\hline 1-hexene & 1.68 & 1.49 & 0.03 & 1.34 & 0.04 & 1.35 & 0.04 \\
\hline 1-hexyne & 0.29 & -0.33 & 0.04 & -0.48 & 0.04 & -0.48 & 0.04 \\
\hline 1-methylcyclohexene & 0.68 & 1.11 & 0.04 & 0.99 & 0.05 & 0.99 & 0.05 \\
\hline 1-nitropropane & -3.34 & -4.27 & 0.03 & -4.38 & 0.03 & -4.39 & 0.03 \\
\hline 1-octene & 2.20 & 1.79 & 0.04 & 1.62 & 0.06 & 1.63 & 0.05 \\
\hline 1-octyne & 0.72 & -0.01 & 0.05 & -0.23 & 0.07 & -0.21 & 0.07 \\
\hline 1-pentanol & -4.47 & -2.63 & 0.12 & -3.89 & 0.10 & -5.00 & 0.31 \\
\hline 1-pentene & 1.66 & 1.46 & 0.03 & 1.37 & 0.03 & 1.36 & 0.03 \\
\hline 1-pentyne & 0.01 & -0.50 & 0.02 & -0.60 & 0.03 & -0.59 & 0.03 \\
\hline 1-propanol & -4.83 & -3.19 & 0.13 & -4.06 & 0.10 & -4.46 & 0.14 \\
\hline 2,2,2-trifluoroethanol & -4.31 & -3.10 & 0.07 & -3.53 & 0.07 & -3.67 & 0.07 \\
\hline 2,2,4-trimethylpentane & 2.85 & 1.91 & 0.03 & 1.79 & 0.03 & 1.79 & 0.03 \\
\hline 2-butanol & -4.58 & -2.35 & 0.12 & -3.72 & 0.12 & -5.33 & 0.32 \\
\hline 2-butanone & -3.64 & -3.11 & 0.03 & -3.25 & 0.03 & -3.24 & 0.03 \\
\hline 2-methyl-1-propanol & -4.52 & -2.27 & 0.21 & -4.15 & 0.17 & -6.35 & 0.46 \\
\hline 2-methyl-2-butanol & -4.43 & -2.57 & 0.11 & -3.47 & 0.12 & -3.78 & 0.16 \\
\hline 2-pentanone & -3.53 & -3.46 & 0.03 & -3.59 & 0.03 & -3.59 & 0.03 \\
\hline 2-propanol & -4.76 & -3.00 & 0.16 & -3.97 & 0.11 & -4.88 & 0.28 \\
\hline 3-methylpentane & 2.51 & 2.27 & 0.03 & 2.18 & 0.04 & 2.18 & 0.04 \\
\hline 3-pentanone & -3.41 & -2.63 & 0.04 & -2.81 & 0.05 & -2.81 & 0.05 \\
\hline acetone & -3.85 & -3.49 & 0.03 & -3.59 & 0.04 & -3.59 & 0.04 \\
\hline acetonitrile & -3.89 & -3.86 & 0.02 & -3.92 & 0.02 & -3.92 & 0.02 \\
\hline benzene & -0.87 & -1.23 & 0.03 & -1.36 & 0.04 & -1.36 & 0.04 \\
\hline butanal & -3.18 & -3.12 & 0.02 & -3.21 & 0.03 & -3.21 & 0.03 \\
\hline carbon_tetrachloride & 0.10 & 1.48 & 0.04 & 1.37 & 0.04 & 1.37 & 0.04 \\
\hline chloroform & -1.07 & 0.16 & 0.03 & 0.09 & 0.03 & 0.08 & 0.03 \\
\hline cyclohexane & 1.23 & 1.14 & 0.04 & 1.04 & 0.04 & 1.04 & 0.04 \\
\hline cyclohexanol & -5.47 & -3.77 & 0.16 & -4.91 & 0.09 & -6.07 & 0.22 \\
\hline cyclohexene & 0.37 & 0.82 & 0.04 & 0.71 & 0.05 & 0.71 & 0.04 \\
\hline cyclopentane & 1.20 & 1.33 & 0.03 & 1.24 & 0.03 & 1.24 & 0.03 \\
\hline decane & 3.22 & 3.00 & 0.04 & 2.83 & 0.04 & 2.82 & 0.04 \\
\hline dichloromethane & -1.36 & -0.37 & 0.03 & -0.43 & 0.03 & -0.43 & 0.03 \\
\hline
\end{tabular}




\begin{tabular}{|c|c|c|c|c|c|c|c|}
\hline diethyl_ether & -1.76 & 0.07 & 0.04 & -0.05 & 0.06 & -0.05 & 0.05 \\
\hline diisopropyl_ether & -0.53 & -1.37 & 0.04 & -1.54 & 0.06 & -1.54 & 0.06 \\
\hline dimethyl_ether & -1.92 & -0.33 & 0.03 & -0.39 & 0.03 & -0.40 & 0.03 \\
\hline ethanol & -5.01 & -3.56 & 0.08 & -4.18 & 0.09 & -4.46 & 0.16 \\
\hline ethene & 1.27 & 1.56 & 0.02 & 1.50 & 0.02 & 1.50 & 0.02 \\
\hline ethyl_acetate & -3.10 & -3.58 & 0.05 & -3.77 & 0.07 & -3.76 & 0.06 \\
\hline ethylbenzene & -0.80 & -0.97 & 0.04 & -1.14 & 0.04 & -1.16 & 0.04 \\
\hline heptane & 2.62 & 2.46 & 0.04 & 2.31 & 0.05 & 2.31 & 0.05 \\
\hline hexane & 2.49 & 2.41 & 0.03 & 2.28 & 0.04 & 2.28 & 0.04 \\
\hline isopropylbenzene & -0.30 & -0.98 & 0.04 & -1.14 & 0.04 & -1.15 & 0.04 \\
\hline methane & 2.00 & 2.18 & 0.02 & 2.15 & 0.02 & 2.15 & 0.02 \\
\hline methanol & -5.11 & -3.06 & 0.19 & -3.97 & 0.08 & -4.80 & 0.21 \\
\hline methylcyclohexane & 1.71 & 1.24 & 0.04 & 1.14 & 0.04 & 1.14 & 0.04 \\
\hline methylcyclopentane & 1.60 & 1.33 & 0.05 & 1.23 & 0.04 & 1.23 & 0.05 \\
\hline methyl_tert-butyl_ether & -2.21 & -0.47 & 0.03 & -0.60 & 0.03 & -0.60 & 0.03 \\
\hline m-xylene & -0.84 & -0.75 & 0.03 & -0.91 & 0.03 & -0.91 & 0.03 \\
\hline nitromethane & -3.95 & -4.85 & 0.02 & -4.91 & 0.02 & -4.91 & 0.02 \\
\hline nonane & 3.04 & 2.52 & 0.04 & 2.39 & 0.05 & 2.40 & 0.05 \\
\hline n-pentane & 2.33 & 2.30 & 0.03 & 2.22 & 0.03 & 2.21 & 0.03 \\
\hline octane & 2.89 & 2.61 & 0.05 & 2.46 & 0.05 & 2.45 & 0.05 \\
\hline o-xylene & -0.90 & -0.79 & 0.03 & -0.92 & 0.03 & -0.91 & 0.03 \\
\hline propanal & -3.44 & -2.67 & 0.04 & -2.76 & 0.04 & -2.76 & 0.04 \\
\hline p-xylene & -0.81 & -0.63 & 0.05 & -0.76 & 0.05 & -0.76 & 0.05 \\
\hline pyridine & -4.70 & -2.86 & 0.04 & -3.00 & 0.04 & -3.01 & 0.03 \\
\hline tert-butanol & -4.51 & -3.27 & 0.10 & -3.84 & 0.10 & -4.06 & 0.12 \\
\hline tert-butylbenzene & -0.44 & -1.26 & 0.06 & -1.47 & 0.06 & -1.49 & 0.07 \\
\hline tetrahydrofuran & -3.47 & -2.12 & 0.04 & -2.26 & 0.04 & -2.26 & 0.04 \\
\hline thiophene & -1.42 & -1.43 & 0.03 & -1.55 & 0.04 & -1.55 & 0.04 \\
\hline toluene & -0.89 & -0.87 & 0.03 & -1.04 & 0.03 & -1.04 & 0.03 \\
\hline triethylamine & -3.02 & 1.18 & 0.05 & 1.00 & 0.06 & 0.98 & 0.05 \\
\hline RMSE & & 1.16 & & 0.91 & & 0.89 & \\
\hline MSE & & -0.49 & & -0.20 & & -0.02 & \\
\hline MAE & & 0.86 & & 0.68 & & 0.65 & \\
\hline$\tau$ & & 0.72 & & 0.77 & & 0.78 & \\
\hline PI & & 0.92 & & 0.95 & & 0.95 & \\
\hline
\end{tabular}


Table S3. Solvation free energies in [EMIM][DCA] with the charge scaling factor of $0.6 \mathrm{in} \mathrm{kcal} / \mathrm{mol}$. $\Delta G_{W_{a}}$ is the slow-growth estimate, $\Delta G_{\mathrm{EXP}}$ represents exponential averaging, and $\Delta G_{\mathrm{GEXP}}$ is its Gaussian approximation. SD represents the standard error of the free energy estimate, which is obtained from numerical bootstrap resampling. MSE, MAE, RMSE, $\tau$, and PI serve as quality measurements.

\begin{tabular}{|c|c|c|c|c|c|c|c|}
\hline Solute & Experiment & $\Delta G_{W_{a}}$ & $\mathrm{SD}$ & $\Delta G_{\mathrm{EXP}}$ & $\mathrm{SD}$ & $\Delta G_{\mathrm{GEXP}}$ & $\mathrm{SD}$ \\
\hline 1,4-dioxane & -4.93 & -5.31 & 0.07 & -5.63 & 0.07 & -5.65 & 0.07 \\
\hline 1-butanol & -5.25 & -4.65 & 0.06 & -5.03 & 0.09 & -5.04 & 0.07 \\
\hline 1-heptyne & -2.97 & -5.47 & 0.04 & -5.83 & 0.06 & -5.83 & 0.05 \\
\hline 1-hexene & -1.28 & -2.38 & 0.07 & -2.74 & 0.07 & -2.77 & 0.08 \\
\hline 1-hexyne & -2.63 & -4.78 & 0.07 & -5.07 & 0.07 & -5.11 & 0.07 \\
\hline 1-nitropropane & -5.14 & -6.49 & 0.06 & -6.78 & 0.07 & -6.79 & 0.08 \\
\hline 1-propanol & -4.82 & -4.02 & 0.06 & -4.29 & 0.07 & -4.27 & 0.07 \\
\hline 2,2,2-trifluoroethanol & -5.65 & -4.43 & 0.06 & -4.66 & 0.07 & -4.66 & 0.07 \\
\hline 2,2,4-trimethylpentane & -1.26 & -3.78 & 0.07 & -4.24 & 0.09 & -4.25 & 0.09 \\
\hline 2-butanone & -3.73 & -4.37 & 0.05 & -4.64 & 0.06 & -4.66 & 0.06 \\
\hline 2-methyl-1-propanol & -5.00 & -4.93 & 0.05 & -5.20 & 0.04 & -5.21 & 0.04 \\
\hline 2-pentanone & -4.06 & -5.17 & 0.08 & -5.58 & 0.10 & -5.58 & 0.10 \\
\hline 2-propanol & -4.36 & -3.96 & 0.04 & -4.17 & 0.05 & -4.18 & 0.06 \\
\hline 3-methylpentane & -0.82 & -2.39 & 0.07 & -2.92 & 0.11 & -2.86 & 0.10 \\
\hline 3-pentanone & -4.01 & -4.83 & 0.08 & -5.24 & 0.13 & -5.23 & 0.12 \\
\hline acetone & -3.44 & -3.94 & 0.05 & -4.13 & 0.04 & -4.14 & 0.05 \\
\hline acetonitrile & -4.28 & -4.44 & 0.06 & -4.70 & 0.06 & -4.71 & 0.06 \\
\hline benzene & -3.55 & -4.03 & 0.07 & -4.38 & 0.08 & -4.39 & 0.08 \\
\hline butanal & -3.54 & -4.61 & 0.05 & -4.92 & 0.06 & -4.93 & 0.07 \\
\hline carbon_tetrachloride & -3.30 & -2.73 & 0.06 & -3.05 & 0.08 & -3.08 & 0.09 \\
\hline chloroform & -3.93 & -3.09 & 0.04 & -3.31 & 0.05 & -3.32 & 0.06 \\
\hline cyclohexane & -1.65 & -3.29 & 0.06 & -3.64 & 0.08 & -3.68 & 0.08 \\
\hline decane & -2.36 & -5.24 & 0.06 & -5.58 & 0.09 & -5.59 & 0.09 \\
\hline dichloromethane & -3.41 & -2.91 & 0.05 & -3.07 & 0.05 & -3.07 & 0.05 \\
\hline diethyl_ether & -1.85 & -2.43 & 0.06 & -2.81 & 0.08 & -2.81 & 0.08 \\
\hline diisopropyl_ether & -0.84 & -3.93 & 0.08 & -4.43 & 0.11 & -4.42 & 0.12 \\
\hline dimethyl_ether & -2.03 & -1.40 & 0.04 & -1.53 & 0.04 & -1.53 & 0.04 \\
\hline ethanol & -4.48 & -3.46 & 0.05 & -3.66 & 0.05 & -3.67 & 0.05 \\
\hline ethene & 0.40 & 0.65 & 0.04 & 0.53 & 0.04 & 0.53 & 0.04 \\
\hline ethyl_acetate & -3.41 & -4.71 & 0.07 & -5.04 & 0.08 & -5.04 & 0.08 \\
\hline ethylbenzene & -4.21 & -5.14 & 0.06 & -5.51 & 0.08 & -5.53 & 0.09 \\
\hline heptane & -1.33 & -3.14 & 0.08 & -3.54 & 0.11 & -3.58 & 0.10 \\
\hline hexane & -0.83 & -2.36 & 0.08 & -2.80 & 0.08 & -2.85 & 0.09 \\
\hline methanol & -4.38 & -2.66 & 0.04 & -2.78 & 0.04 & -2.79 & 0.04 \\
\hline methylcyclohexane & -1.85 & -3.86 & 0.07 & -4.26 & 0.10 & -4.25 & 0.10 \\
\hline methylcyclopentane & -1.32 & -3.25 & 0.08 & -3.53 & 0.08 & -3.59 & 0.09 \\
\hline
\end{tabular}




\begin{tabular}{cccccccc} 
m-xylene & -4.04 & -5.44 & 0.09 & -5.93 & 0.11 & -5.93 & 0.11 \\
nitromethane & -4.85 & -5.65 & 0.04 & -5.85 & 0.04 & -5.86 & 0.04 \\
nonane & -1.96 & -4.60 & 0.07 & -5.13 & 0.07 & -5.14 & 0.08 \\
octane & -1.67 & -3.60 & 0.07 & -4.01 & 0.12 & -4.02 & 0.10 \\
o-xylene & -4.59 & -5.42 & 0.09 & -5.82 & 0.07 & -5.90 & 0.07 \\
propanal & -3.20 & -3.57 & 0.04 & -3.74 & 0.05 & -3.74 & 0.05 \\
p-xylene & -4.33 & -5.32 & 0.05 & -5.68 & 0.06 & -5.72 & 0.07 \\
pyridine & -4.94 & -4.87 & 0.05 & -5.06 & 0.06 & -5.07 & 0.07 \\
tetrahydrofuran & -3.23 & -4.04 & 0.07 & -4.36 & 0.09 & -4.39 & 0.09 \\
thiophene & -3.90 & -4.35 & 0.05 & -4.57 & 0.05 & -4.56 & 0.05 \\
toluene & -3.94 & -4.88 & 0.06 & -5.14 & 0.07 & -5.17 & 0.07 \\
triethylamine & -1.93 & -3.50 & 0.06 & -3.85 & 0.07 & -3.85 & 0.07 \\
\hline RMSE & & 1.38 & & 1.64 & & 1.65 & \\
MSE & & 0.79 & & 1.11 & & 1.12 & \\
MAE & & 1.16 & & 1.37 & & 0.38 & \\
$\tau$ & & 0.43 & & 0.40 & & 0.57 & \\
PI & & 0.61 & & 0.57 & & \\
\hline
\end{tabular}


Table S4. Solvation free energies in [EMIM][DCA] with the charge scaling factor of $0.7 \mathrm{in} \mathrm{kcal} / \mathrm{mol}$. $\Delta G_{W_{a}}$ is the slow-growth estimate, $\Delta G_{\mathrm{EXP}}$ represents exponential averaging, and $\Delta G_{\mathrm{GEXP}}$ is its Gaussian approximation. SD represents the standard error of the free energy estimate, which is obtained from numerical bootstrap resampling. MSE, MAE, RMSE, $\tau$, and PI serve as quality measurements.

\begin{tabular}{|c|c|c|c|c|c|c|c|}
\hline Solute & Experiment & $\Delta G_{W_{a}}$ & $\mathrm{SD}$ & $\Delta G_{\mathrm{EXP}}$ & $\mathrm{SD}$ & $\Delta G_{\mathrm{GEXP}}$ & $\mathrm{SD}$ \\
\hline 1,4-dioxane & -4.93 & -4.87 & 0.09 & -5.45 & 0.10 & -5.50 & 0.11 \\
\hline 1-butanol & -5.25 & -4.68 & 0.07 & -5.16 & 0.11 & -5.16 & 0.07 \\
\hline 1-heptyne & -2.97 & -4.73 & 0.10 & -5.40 & 0.15 & -5.36 & 0.15 \\
\hline 1-hexene & -1.28 & -1.68 & 0.07 & -2.08 & 0.06 & -2.10 & 0.06 \\
\hline 1-hexyne & -2.63 & -4.12 & 0.09 & -4.71 & 0.11 & -4.72 & 0.12 \\
\hline 1-nitropropane & -5.14 & -6.19 & 0.07 & -6.81 & 0.13 & -6.82 & 0.13 \\
\hline 1-propanol & -4.82 & -3.95 & 0.06 & -4.24 & 0.07 & -4.24 & 0.07 \\
\hline 2,2,2-trifluoroethanol & -5.65 & -4.82 & 0.07 & -5.36 & 0.12 & -5.36 & 0.13 \\
\hline 2,2,4-trimethylpentane & -1.26 & -2.66 & 0.14 & -3.94 & 0.29 & -3.84 & 0.29 \\
\hline 2-butanone & -3.73 & -3.99 & 0.07 & -4.58 & 0.11 & -4.56 & 0.11 \\
\hline 2-methyl-1-propanol & -5.00 & -4.90 & 0.07 & -5.56 & 0.15 & -5.45 & 0.13 \\
\hline 2-pentanone & -4.06 & -4.73 & 0.13 & -5.44 & 0.19 & -5.46 & 0.18 \\
\hline 2-propanol & -4.36 & -4.04 & 0.06 & -4.49 & 0.11 & -4.43 & 0.10 \\
\hline 3-methylpentane & -0.82 & -1.47 & 0.09 & -2.00 & 0.08 & -2.07 & 0.09 \\
\hline 3-pentanone & -4.01 & -4.11 & 0.10 & -4.63 & 0.11 & -4.67 & 0.12 \\
\hline acetone & -3.44 & -3.74 & 0.05 & -4.10 & 0.06 & -4.11 & 0.06 \\
\hline acetonitrile & -4.28 & -4.21 & 0.06 & -4.53 & 0.09 & -4.53 & 0.09 \\
\hline benzene & -3.55 & -3.42 & 0.10 & -3.90 & 0.09 & -3.96 & 0.11 \\
\hline butanal & -3.54 & -4.12 & 0.07 & -4.65 & 0.12 & -4.61 & 0.12 \\
\hline carbon_tetrachloride & -3.30 & -2.00 & 0.08 & -2.67 & 0.14 & -2.63 & 0.14 \\
\hline chloroform & -3.93 & -2.81 & 0.06 & -3.16 & 0.09 & -3.13 & 0.08 \\
\hline cyclohexane & -1.65 & -2.44 & 0.09 & -3.15 & 0.16 & -3.06 & 0.16 \\
\hline decane & -2.36 & -3.37 & 0.13 & -4.44 & 0.16 & -4.76 & 0.21 \\
\hline dichloromethane & -3.41 & -2.65 & 0.05 & -2.94 & 0.05 & -2.96 & 0.05 \\
\hline diethyl_ether & -1.85 & -2.06 & 0.08 & -2.49 & 0.10 & -2.51 & 0.11 \\
\hline diisopropyl_ether & -0.84 & -3.13 & 0.10 & -3.72 & 0.11 & -3.80 & 0.13 \\
\hline dimethyl_ether & -2.03 & -1.00 & 0.04 & -1.21 & 0.05 & -1.22 & 0.05 \\
\hline ethanol & -4.48 & -3.59 & 0.06 & -3.88 & 0.08 & -3.88 & 0.08 \\
\hline ethene & 0.40 & 0.91 & 0.05 & 0.77 & 0.05 & 0.77 & 0.05 \\
\hline ethyl_acetate & -3.41 & -4.27 & 0.07 & -4.65 & 0.05 & -4.69 & 0.07 \\
\hline ethylbenzene & -4.21 & -4.47 & 0.08 & -5.06 & 0.11 & -5.16 & 0.13 \\
\hline heptane & -1.33 & -2.06 & 0.10 & -3.04 & 0.16 & -2.97 & 0.15 \\
\hline hexane & -0.83 & -1.63 & 0.07 & -2.10 & 0.08 & -2.12 & 0.10 \\
\hline methanol & -4.38 & -3.00 & 0.04 & -3.21 & 0.05 & -3.23 & 0.05 \\
\hline methylcyclohexane & -1.85 & -2.91 & 0.09 & -3.62 & 0.11 & -3.69 & 0.14 \\
\hline methylcyclopentane & -1.32 & -2.42 & 0.07 & -2.94 & 0.12 & -2.99 & 0.12 \\
\hline
\end{tabular}




\begin{tabular}{cccccccc} 
m-xylene & -4.04 & -4.52 & 0.10 & -5.25 & 0.12 & -5.39 & 0.15 \\
nitromethane & -4.85 & -5.74 & 0.09 & -6.16 & 0.10 & -6.19 & 0.09 \\
nonane & -1.96 & -3.20 & 0.10 & -4.21 & 0.17 & -4.24 & 0.18 \\
octane & -1.67 & -2.46 & 0.09 & -3.26 & 0.12 & -3.32 & 0.15 \\
o-xylene & -4.59 & -4.67 & 0.10 & -5.50 & 0.11 & -5.55 & 0.15 \\
propanal & -3.20 & -3.30 & 0.06 & -3.66 & 0.08 & -3.70 & 0.10 \\
p-xylene & -4.33 & -4.52 & 0.10 & -5.21 & 0.12 & -5.19 & 0.13 \\
pyridine & -4.94 & -4.56 & 0.08 & -5.01 & 0.09 & -5.04 & 0.09 \\
tetrahydrofuran & -3.23 & -3.70 & 0.07 & -4.11 & 0.07 & -4.15 & 0.07 \\
thiophene & -3.90 & -4.06 & 0.07 & -4.55 & 0.11 & -4.50 & 0.10 \\
toluene & -3.94 & -4.13 & 0.08 & -4.67 & 0.09 & -4.73 & 0.09 \\
triethylamine & -1.93 & -2.41 & 0.09 & -3.00 & 0.09 & -3.05 & 0.12 \\
\hline RMSE & & 0.85 & & 1.25 & & 1.27 & \\
MSE & & 0.26 & & 0.81 & & 0.83 & \\
MAE & & 0.69 & & 1.05 & & 0.07 & \\
$\tau$ & & 0.61 & & 0.55 & & 0.72 & \\
PI & & 0.78 & & 0.73 & & \\
\hline
\end{tabular}


Table S5. Solvation free energies in [EMIM][DCA] with the charge scaling factor of $0.8 \mathrm{in} \mathrm{kcal} / \mathrm{mol}$. $\Delta G_{W_{a}}$ is the slow-growth estimate, $\Delta G_{\mathrm{EXP}}$ represents exponential averaging, and $\Delta G_{\mathrm{GEXP}}$ is its Gaussian approximation. SD represents the standard error of the free energy estimate, which is obtained from numerical bootstrap resampling. MSE, MAE, RMSE, $\tau$, and PI serve as quality measurements.

\begin{tabular}{|c|c|c|c|c|c|c|c|}
\hline Solute & Experiment & $\Delta G_{W_{a}}$ & SD & $\Delta G_{\mathrm{EXP}}$ & $\mathrm{SD}$ & $\Delta G_{\mathrm{GEXP}}$ & $\mathrm{SD}$ \\
\hline 1,4-dioxane & -4.93 & -3.86 & 0.12 & -4.92 & 0.11 & -5.04 & 0.14 \\
\hline 1-butanol & -5.25 & -4.09 & 0.13 & -5.40 & 0.19 & -5.39 & 0.20 \\
\hline 1-heptyne & -2.97 & -3.52 & 0.13 & -4.70 & 0.21 & -4.69 & 0.20 \\
\hline 1-hexene & -1.28 & 0.01 & 0.10 & -1.31 & 0.20 & -1.38 & 0.22 \\
\hline 1-hexyne & -2.63 & -2.59 & 0.14 & -4.00 & 0.21 & -4.14 & 0.27 \\
\hline 1-nitropropane & -5.14 & -5.68 & 0.08 & -6.27 & 0.09 & -6.41 & 0.14 \\
\hline 1-propanol & -4.82 & -4.03 & 0.10 & -4.54 & 0.15 & -4.55 & 0.15 \\
\hline 2,2,2-trifluoroethanol & -5.65 & -4.71 & 0.11 & -5.50 & 0.10 & -5.72 & 0.15 \\
\hline 2,2,4-trimethylpentane & -1.26 & -1.35 & 0.11 & -2.59 & 0.16 & -2.81 & 0.20 \\
\hline 2-butanone & -3.73 & -2.77 & 0.12 & -3.88 & 0.14 & -4.02 & 0.19 \\
\hline 2-methyl-1-propanol & -5.00 & -4.20 & 0.10 & -5.46 & 0.13 & -5.56 & 0.19 \\
\hline 2-pentanone & -4.06 & -3.38 & 0.10 & -4.38 & 0.26 & -4.65 & 0.20 \\
\hline 2-propanol & -4.36 & -3.55 & 0.09 & -4.49 & 0.14 & -4.64 & 0.20 \\
\hline 3-methylpentane & -0.82 & 0.41 & 0.17 & -1.42 & 0.42 & -1.51 & 0.36 \\
\hline 3-pentanone & -4.01 & -2.78 & 0.10 & -3.97 & 0.15 & -4.19 & 0.15 \\
\hline acetone & -3.44 & -2.82 & 0.10 & -3.65 & 0.12 & -3.69 & 0.13 \\
\hline acetonitrile & -4.28 & -3.55 & 0.08 & -4.26 & 0.11 & -4.33 & 0.09 \\
\hline benzene & -3.55 & -2.14 & 0.11 & -3.21 & 0.16 & -3.52 & 0.20 \\
\hline butanal & -3.54 & -3.11 & 0.09 & -4.30 & 0.15 & -4.34 & 0.17 \\
\hline carbon_tetrachloride & -3.30 & -0.35 & 0.13 & -1.81 & 0.24 & -1.83 & 0.26 \\
\hline chloroform & -3.93 & -2.04 & 0.09 & -2.79 & 0.10 & -3.00 & 0.14 \\
\hline cyclohexane & -1.65 & -0.49 & 0.11 & -2.10 & 0.13 & -2.23 & 0.18 \\
\hline decane & -2.36 & -0.64 & 0.19 & -3.06 & 0.39 & -3.75 & 0.44 \\
\hline dichloromethane & -3.41 & -1.94 & 0.07 & -2.81 & 0.10 & -2.77 & 0.12 \\
\hline diethyl_ether & -1.85 & -0.67 & 0.12 & -1.69 & 0.14 & -1.96 & 0.21 \\
\hline diisopropyl_ether & -0.84 & -1.05 & 0.13 & -2.31 & 0.19 & -2.72 & 0.21 \\
\hline dimethyl_ether & -2.03 & -0.13 & 0.08 & -0.72 & 0.09 & -0.76 & 0.10 \\
\hline ethanol & -4.48 & -3.37 & 0.10 & -4.16 & 0.12 & -4.29 & 0.16 \\
\hline ethene & 0.40 & 1.61 & 0.07 & 1.17 & 0.08 & 1.20 & 0.07 \\
\hline ethyl_acetate & -3.41 & -3.11 & 0.14 & -4.14 & 0.19 & -4.34 & 0.25 \\
\hline ethylbenzene & -4.21 & -2.96 & 0.12 & -4.37 & 0.16 & -4.71 & 0.25 \\
\hline heptane & -1.33 & -0.22 & 0.15 & -1.82 & 0.24 & -2.38 & 0.35 \\
\hline hexane & -0.83 & 0.09 & 0.11 & -1.08 & 0.25 & -1.02 & 0.23 \\
\hline methanol & -4.38 & -2.99 & 0.08 & -3.43 & 0.09 & -3.49 & 0.09 \\
\hline methylcyclohexane & -1.85 & -0.89 & 0.12 & -2.77 & 0.25 & -3.00 & 0.39 \\
\hline methylcyclopentane & -1.32 & -0.93 & 0.16 & -2.51 & 0.22 & -2.93 & 0.23 \\
\hline
\end{tabular}




\begin{tabular}{cccccccc} 
m-xylene & -4.04 & -2.94 & 0.17 & -4.23 & 0.21 & -4.56 & 0.25 \\
nitromethane & -4.85 & -4.93 & 0.09 & -5.67 & 0.16 & -5.65 & 0.16 \\
nonane & -1.96 & -0.28 & 0.20 & -2.28 & 0.39 & -3.10 & 0.36 \\
octane & -1.67 & 0.10 & 0.15 & -1.66 & 0.19 & -1.96 & 0.32 \\
o-xylene & -4.59 & -2.99 & 0.20 & -4.53 & 0.18 & -5.26 & 0.28 \\
propanal & -3.20 & -2.43 & 0.10 & -3.20 & 0.14 & -3.25 & 0.16 \\
p-xylene & -4.33 & -3.08 & 0.20 & -4.90 & 0.23 & -5.51 & 0.27 \\
pyridine & -4.94 & -3.57 & 0.14 & -4.83 & 0.25 & -4.91 & 0.26 \\
tetrahydrofuran & -3.23 & -2.55 & 0.10 & -3.50 & 0.16 & -3.49 & 0.17 \\
thiophene & -3.90 & -2.96 & 0.13 & -3.98 & 0.11 & -4.29 & 0.20 \\
toluene & -3.94 & -2.56 & 0.17 & -4.38 & 0.34 & -4.46 & 0.35 \\
triethylamine & -1.93 & -0.64 & 0.21 & -2.25 & 0.35 & -2.70 & 0.37 \\
\hline RMSE & & 1.18 & & 0.71 & & 0.86 & \\
MSE & & -0.99 & & 0.21 & & 0.41 & \\
MAE & & 1.05 & & 0.53 & & 0.68 & \\
$\tau$ & & 0.70 & & 0.69 & & 0.67 & \\
PI & & 0.90 & & 0.85 & & 0.83 & \\
\hline
\end{tabular}


Table S6. Solvation free energies in [EMIM][NTf2] with the charge scaling factor of $0.6 \mathrm{in} \mathrm{kcal} / \mathrm{mol}$. $\Delta G_{W_{a}}$ is the slow-growth estimate, $\Delta G_{\mathrm{EXP}}$ represents exponential averaging, and $\Delta G_{\mathrm{GEXP}}$ is its Gaussian approximation. SD represents the standard error of the free energy estimate, which is obtained from numerical bootstrap resampling. MSE, MAE, RMSE, $\tau$, and PI serve as quality measurements.

\begin{tabular}{|c|c|c|c|c|c|c|c|}
\hline Solute & Experiment & $\Delta G_{W_{a}}$ & $\mathrm{SD}$ & $\Delta G_{\mathrm{EXP}}$ & $\mathrm{SD}$ & $\Delta G_{\mathrm{GEXP}}$ & $\mathrm{SD}$ \\
\hline 1-butanol & -4.57 & -3.55 & 0.08 & -4.17 & 0.14 & -4.12 & 0.16 \\
\hline 1-hexanol & -5.47 & -4.59 & 0.11 & -5.50 & 0.17 & -5.59 & 0.17 \\
\hline 1-methylcyclohexene & -3.07 & -3.12 & 0.09 & -4.07 & 0.13 & -4.10 & 0.14 \\
\hline 1-pentanol & -4.82 & -4.18 & 0.10 & -5.44 & 0.17 & -5.61 & 0.23 \\
\hline 1-propanol & -4.29 & -2.89 & 0.09 & -3.57 & 0.14 & -3.61 & 0.15 \\
\hline 2,2,4-trimethylpentane & -2.18 & -2.89 & 0.19 & -4.47 & 0.17 & -4.87 & 0.24 \\
\hline 2-butanol & -4.08 & -3.10 & 0.09 & -3.87 & 0.17 & -3.88 & 0.12 \\
\hline 2-methyl-2-butanol & -4.23 & -3.00 & 0.16 & -3.99 & 0.20 & -4.11 & 0.20 \\
\hline 2-propanol & -3.71 & -2.73 & 0.10 & -3.47 & 0.13 & -3.51 & 0.14 \\
\hline acetone & -3.87 & -3.13 & 0.08 & -3.76 & 0.12 & -3.84 & 0.13 \\
\hline acetonitrile & -4.36 & -3.47 & 0.07 & -4.06 & 0.10 & -4.06 & 0.12 \\
\hline benzene & -3.85 & -3.30 & 0.08 & -4.06 & 0.10 & -4.31 & 0.16 \\
\hline carbon_tetrachloride & -3.02 & -2.39 & 0.10 & -3.30 & 0.13 & -3.52 & 0.21 \\
\hline chloroform & -3.46 & -2.66 & 0.07 & -3.31 & 0.08 & -3.44 & 0.12 \\
\hline cyclohexane & -2.28 & -2.72 & 0.08 & -3.43 & 0.11 & -3.46 & 0.12 \\
\hline cyclohexanol & -5.94 & -5.20 & 0.12 & -6.62 & 0.16 & -7.16 & 0.27 \\
\hline cyclohexene & -2.73 & -2.70 & 0.12 & -3.53 & 0.11 & -3.61 & 0.16 \\
\hline decane & -3.25 & -3.80 & 0.17 & -5.13 & 0.21 & -5.68 & 0.28 \\
\hline dichloromethane & -3.08 & -2.34 & 0.07 & -2.87 & 0.08 & -2.94 & 0.10 \\
\hline ethanol & -3.76 & -2.22 & 0.07 & -2.72 & 0.10 & -2.73 & 0.11 \\
\hline ethyl_acetate & -4.01 & -3.75 & 0.12 & -4.95 & 0.21 & -4.83 & 0.16 \\
\hline ethylbenzene & -4.67 & -4.19 & 0.11 & -5.01 & 0.15 & -5.15 & 0.22 \\
\hline heptane & -2.20 & -2.46 & 0.13 & -3.68 & 0.27 & -3.70 & 0.18 \\
\hline hexane & -1.69 & -2.18 & 0.13 & -3.13 & 0.16 & -3.29 & 0.18 \\
\hline isopropylbenzene & -4.86 & -5.23 & 0.12 & -6.23 & 0.17 & -6.28 & 0.17 \\
\hline methane & 1.08 & 1.37 & 0.05 & 1.17 & 0.06 & 1.15 & 0.06 \\
\hline methanol & -3.63 & -1.60 & 0.04 & -1.85 & 0.05 & -1.88 & 0.05 \\
\hline methyl_tert-butyl_ether & -2.67 & -2.31 & 0.11 & -3.37 & 0.14 & -3.52 & 0.20 \\
\hline m-xylene & -4.78 & -4.00 & 0.21 & -5.58 & 0.19 & -5.94 & 0.28 \\
\hline nonane & -2.90 & -3.58 & 0.13 & -4.95 & 0.15 & -5.28 & 0.25 \\
\hline octane & -2.57 & -3.06 & 0.12 & -4.14 & 0.12 & -4.47 & 0.22 \\
\hline o-xylene & -4.99 & -4.44 & 0.11 & -5.91 & 0.21 & -5.94 & 0.20 \\
\hline p-xylene & -4.76 & -4.29 & 0.13 & -5.62 & 0.21 & -5.90 & 0.20 \\
\hline tert-butanol & -3.81 & -3.27 & 0.11 & -4.38 & 0.18 & -4.45 & 0.17 \\
\hline tert-butylbenzene & -5.17 & -4.96 & 0.13 & -6.48 & 0.16 & -7.04 & 0.29 \\
\hline toluene & -4.32 & -3.75 & 0.17 & -5.08 & 0.22 & -5.21 & 0.22 \\
\hline
\end{tabular}




\begin{tabular}{cccc} 
RMSE & 0.79 & 1.02 & 1.18 \\
MSE & -0.45 & 0.52 & 0.66 \\
MAE & 0.68 & 0.82 & 0.94 \\
$\tau$ & 0.63 & 0.53 & 0.50 \\
PI & 0.85 & 0.76 & 0.73 \\
\hline
\end{tabular}


Table S7. Solvation free energies in [EMIM][NTf2] with the charge scaling factor of $0.7 \mathrm{in} \mathrm{kcal} / \mathrm{mol}$. $\Delta G_{W_{a}}$ is the slow-growth estimate, $\Delta G_{\mathrm{EXP}}$ represents exponential averaging, and $\Delta G_{\mathrm{GEXP}}$ is its Gaussian approximation. SD represents the standard error of the free energy estimate, which is obtained from numerical bootstrap resampling. MSE, MAE, RMSE, $\tau$, and PI serve as quality measurements.

\begin{tabular}{|c|c|c|c|c|c|c|c|}
\hline Solute & Experiment & $\Delta G_{W_{a}}$ & $\mathrm{SD}$ & $\Delta G_{\mathrm{EXP}}$ & $\mathrm{SD}$ & $\Delta G_{\mathrm{GEXP}}$ & $\mathrm{SD}$ \\
\hline 1-butanol & -4.57 & -3.25 & 0.14 & -4.75 & 0.16 & -4.95 & 0.23 \\
\hline 1-hexanol & -5.47 & -4.69 & 0.14 & -5.92 & 0.26 & -6.01 & 0.25 \\
\hline 1-methylcyclohexene & -3.07 & -2.21 & 0.16 & -4.15 & 0.24 & -4.68 & 0.40 \\
\hline 1-pentanol & -4.82 & -3.75 & 0.21 & -5.13 & 0.23 & -5.69 & 0.33 \\
\hline 1-propanol & -4.29 & -2.59 & 0.16 & -3.81 & 0.17 & -4.09 & 0.20 \\
\hline 2,2,4-trimethylpentane & -2.18 & -2.62 & 0.15 & -4.65 & 0.38 & -4.90 & 0.29 \\
\hline 2-butanol & -4.08 & -2.85 & 0.15 & -4.28 & 0.14 & -4.34 & 0.20 \\
\hline 2-methyl-2-butanol & -4.23 & -3.06 & 0.16 & -5.03 & 0.32 & -5.37 & 0.25 \\
\hline 2-propanol & -3.71 & -2.76 & 0.10 & -3.84 & 0.12 & -4.11 & 0.15 \\
\hline acetone & -3.87 & -2.94 & 0.10 & -4.17 & 0.22 & -4.22 & 0.23 \\
\hline acetonitrile & -4.36 & -3.30 & 0.11 & -3.93 & 0.11 & -4.02 & 0.15 \\
\hline benzene & -3.85 & -2.65 & 0.11 & -4.09 & 0.20 & -4.41 & 0.31 \\
\hline carbon_tetrachloride & -3.02 & -1.61 & 0.13 & -3.18 & 0.30 & -3.42 & 0.33 \\
\hline chloroform & -3.46 & -2.36 & 0.15 & -3.32 & 0.18 & -3.52 & 0.17 \\
\hline cyclohexane & -2.28 & -1.28 & 0.12 & -3.78 & 0.27 & -4.02 & 0.33 \\
\hline cyclohexanol & -5.94 & -4.51 & 0.12 & -6.44 & 0.30 & -7.00 & 0.44 \\
\hline cyclohexene & -2.73 & -1.75 & 0.14 & -2.97 & 0.21 & -3.20 & 0.25 \\
\hline decane & -3.25 & -3.56 & 0.15 & -5.09 & 0.24 & -5.62 & 0.33 \\
\hline dichloromethane & -3.08 & -1.92 & 0.13 & -2.97 & 0.17 & -3.04 & 0.19 \\
\hline ethanol & -3.76 & -2.46 & 0.10 & -3.22 & 0.18 & -3.16 & 0.17 \\
\hline ethyl_acetate & -4.01 & -3.05 & 0.17 & -4.58 & 0.34 & -4.53 & 0.33 \\
\hline ethylbenzene & -4.67 & -3.20 & 0.25 & -5.64 & 0.43 & -5.98 & 0.45 \\
\hline heptane & -2.20 & -1.34 & 0.18 & -4.27 & 0.55 & -4.41 & 0.60 \\
\hline hexane & -1.69 & -0.87 & 0.20 & -2.95 & 0.26 & -2.95 & 0.37 \\
\hline isopropylbenzene & -4.86 & -4.54 & 0.17 & -5.16 & 0.23 & -5.75 & 0.30 \\
\hline methane & 1.08 & 1.65 & 0.07 & 1.37 & 0.07 & 1.32 & 0.07 \\
\hline methanol & -3.63 & -1.75 & 0.11 & -2.30 & 0.12 & -2.37 & 0.13 \\
\hline methyl_tert-butyl_ether & -2.67 & -1.42 & 0.13 & -3.42 & 0.14 & -3.62 & 0.25 \\
\hline m-xylene & -4.78 & -3.99 & 0.16 & -5.48 & 0.18 & -5.72 & 0.23 \\
\hline nonane & -2.90 & -3.12 & 0.13 & -4.36 & 0.10 & -5.13 & 0.20 \\
\hline octane & -2.57 & -1.57 & 0.22 & -3.89 & 0.36 & -4.31 & 0.45 \\
\hline o-xylene & -4.99 & -3.99 & 0.17 & -5.28 & 0.13 & -5.67 & 0.21 \\
\hline p-xylene & -4.76 & -4.18 & 0.09 & -5.42 & 0.14 & -5.61 & 0.18 \\
\hline tert-butanol & -3.81 & -3.04 & 0.17 & -5.06 & 0.22 & -5.28 & 0.32 \\
\hline tert-butylbenzene & -5.17 & -4.86 & 0.16 & -6.27 & 0.27 & -7.32 & 0.33 \\
\hline toluene & -4.32 & -3.67 & 0.15 & -4.64 & 0.14 & -4.95 & 0.21 \\
\hline
\end{tabular}




\begin{tabular}{cccc} 
RMSE & 1.04 & 0.95 & 1.21 \\
MSE & -0.91 & 0.56 & 0.84 \\
MAE & 0.97 & 0.74 & 0.98 \\
$\tau$ & 0.75 & 0.57 & 0.53 \\
PI & 0.92 & 0.79 & 0.76 \\
\hline
\end{tabular}


Table S8. Solvation free energies in [EMIM][NTf2] with the charge scaling factor of $0.8 \mathrm{in} \mathrm{kcal} / \mathrm{mol}$. $\Delta G_{W_{a}}$ is the slow-growth estimate, $\Delta G_{\mathrm{EXP}}$ represents exponential averaging, and $\Delta G_{\mathrm{GEXP}}$ is its Gaussian approximation. SD represents the standard error of the free energy estimate, which is obtained from numerical bootstrap resampling. MSE, MAE, RMSE, $\tau$, and PI serve as quality measurements.

\begin{tabular}{|c|c|c|c|c|c|c|c|}
\hline Solute & Experiment & $\Delta G_{W_{a}}$ & $\mathrm{SD}$ & $\Delta G_{\mathrm{EXP}}$ & SD & $\Delta G_{\mathrm{GEXP}}$ & $\mathrm{SD}$ \\
\hline 1-butanol & -4.57 & -3.05 & 0.12 & -4.47 & 0.21 & -4.67 & 0.17 \\
\hline 1-hexanol & -5.47 & -3.58 & 0.16 & -5.78 & 0.47 & -5.93 & 0.45 \\
\hline 1-methylcyclohexene & -3.07 & -1.59 & 0.21 & -3.38 & 0.28 & -3.83 & 0.43 \\
\hline 1-pentanol & -4.82 & -3.19 & 0.14 & -5.30 & 0.38 & -5.65 & 0.39 \\
\hline 1-propanol & -4.29 & -2.56 & 0.10 & -3.61 & 0.22 & -3.69 & 0.13 \\
\hline 2,2,4-trimethylpentane & -2.18 & 0.02 & 0.20 & -2.27 & 0.17 & -3.75 & 0.53 \\
\hline 2-butanol & -4.08 & -2.42 & 0.13 & -3.78 & 0.37 & -3.88 & 0.26 \\
\hline 2-methyl-2-butanol & -4.23 & -2.40 & 0.14 & -4.15 & 0.15 & -4.95 & 0.33 \\
\hline 2-propanol & -3.71 & -2.58 & 0.12 & -3.74 & 0.15 & -3.96 & 0.18 \\
\hline acetone & -3.87 & -2.61 & 0.11 & -4.09 & 0.25 & -4.24 & 0.27 \\
\hline acetonitrile & -4.36 & -3.33 & 0.13 & -4.28 & 0.15 & -4.43 & 0.18 \\
\hline benzene & -3.85 & -2.14 & 0.16 & -3.76 & 0.13 & -4.35 & 0.27 \\
\hline carbon_tetrachloride & -3.02 & -0.95 & 0.14 & -3.08 & 0.39 & -2.98 & 0.28 \\
\hline chloroform & -3.46 & -1.66 & 0.09 & -2.56 & 0.13 & -2.79 & 0.19 \\
\hline cyclohexane & -2.28 & -0.99 & 0.14 & -3.18 & 0.22 & -3.69 & 0.31 \\
\hline cyclohexanol & -5.94 & -3.96 & 0.23 & -6.36 & 0.46 & -7.31 & 0.52 \\
\hline cyclohexene & -2.73 & -1.04 & 0.20 & -2.61 & 0.40 & -3.20 & 0.35 \\
\hline decane & -3.25 & -0.13 & 0.23 & -2.72 & 0.32 & -3.31 & 0.52 \\
\hline dichloromethane & -3.08 & -1.98 & 0.14 & -3.07 & 0.23 & -3.04 & 0.22 \\
\hline ethanol & -3.76 & -2.58 & 0.06 & -3.41 & 0.09 & -3.61 & 0.10 \\
\hline ethyl_acetate & -4.01 & -2.74 & 0.18 & -4.60 & 0.28 & -5.20 & 0.40 \\
\hline ethylbenzene & -4.67 & -2.13 & 0.18 & -4.63 & 0.49 & -4.83 & 0.45 \\
\hline heptane & -2.20 & -0.04 & 0.20 & -1.62 & 0.15 & -2.63 & 0.27 \\
\hline hexane & -1.69 & -0.02 & 0.14 & -1.57 & 0.32 & -1.74 & 0.33 \\
\hline isopropylbenzene & -4.86 & -2.65 & 0.22 & -5.35 & 0.31 & -6.82 & 0.56 \\
\hline methane & 1.08 & 1.79 & 0.08 & 1.47 & 0.09 & 1.47 & 0.09 \\
\hline methanol & -3.63 & -1.88 & 0.07 & -2.52 & 0.13 & -2.53 & 0.15 \\
\hline methyl_tert-butyl_ether & -2.67 & -0.83 & 0.11 & -3.25 & 0.46 & -3.84 & 0.45 \\
\hline m-xylene & -4.78 & -2.45 & 0.15 & -4.61 & 0.33 & -4.84 & 0.36 \\
\hline nonane & -2.90 & -0.54 & 0.22 & -2.89 & 0.14 & -3.70 & 0.40 \\
\hline octane & -2.57 & -0.47 & 0.19 & -3.02 & 0.14 & -3.64 & 0.51 \\
\hline o-xylene & -4.99 & -2.51 & 0.19 & -4.92 & 0.36 & -5.66 & 0.34 \\
\hline p-xylene & -4.76 & -2.43 & 0.18 & -4.42 & 0.22 & -5.01 & 0.30 \\
\hline tert-butanol & -3.81 & -2.69 & 0.24 & -4.58 & 0.32 & -5.00 & 0.38 \\
\hline tert-butylbenzene & -5.17 & -2.64 & 0.18 & -5.09 & 0.23 & -6.18 & 0.37 \\
\hline toluene & -4.32 & -2.31 & 0.16 & -3.83 & 0.18 & -4.33 & 0.24 \\
\hline
\end{tabular}




\begin{tabular}{cccc}
\hline RMSE & 1.87 & 0.45 & 0.80 \\
MSE & -1.80 & -0.03 & 0.44 \\
MAE & 1.80 & 0.34 & 0.62 \\
$\tau$ & 0.69 & 0.77 & 0.67 \\
PI & 0.92 & 0.94 & 0.90 \\
\hline
\end{tabular}


Table S9. Solvation free energies in [EMIM][OTf] with the charge scaling factor of $0.6 \mathrm{in} \mathrm{kcal} / \mathrm{mol} . \Delta G_{W_{a}}$ is the slow-growth estimate, $\Delta G_{\mathrm{EXP}}$ represents exponential averaging, and $\Delta G_{\mathrm{GEXP}}$ is its Gaussian approximation. SD represents the standard error of the free energy estimate, which is obtained from numerical bootstrap resampling. MSE, MAE, RMSE, $\tau$, and PI serve as quality measurements.

\begin{tabular}{|c|c|c|c|c|c|c|c|}
\hline Solute & Experiment & $\Delta G_{W_{a}}$ & $\mathrm{SD}$ & $\Delta G_{\mathrm{EXP}}$ & $\mathrm{SD}$ & $\Delta G_{\mathrm{GEXP}}$ & $\mathrm{SD}$ \\
\hline 1-butanol & -5.41 & -3.92 & 0.09 & -4.53 & 0.09 & -4.61 & 0.10 \\
\hline 1-heptyne & -3.60 & -4.74 & 0.14 & -5.58 & 0.17 & -5.61 & 0.13 \\
\hline 1-hexene & -1.92 & -1.95 & 0.09 & -2.66 & 0.10 & -2.72 & 0.13 \\
\hline 1-hexyne & -3.16 & -4.26 & 0.10 & -4.90 & 0.11 & -5.06 & 0.10 \\
\hline 1-octene & -2.73 & -2.85 & 0.12 & -3.79 & 0.13 & -3.86 & 0.19 \\
\hline 1-octyne & -4.06 & -5.17 & 0.12 & -5.99 & 0.12 & -6.11 & 0.16 \\
\hline 1-pentene & -1.45 & -1.29 & 0.11 & -1.98 & 0.15 & -1.97 & 0.15 \\
\hline 1-pentyne & -2.64 & -3.69 & 0.07 & -4.31 & 0.09 & -4.31 & 0.10 \\
\hline 1-propanol & -4.82 & -3.42 & 0.07 & -3.92 & 0.08 & -3.94 & 0.09 \\
\hline benzene & -3.87 & -3.55 & 0.08 & -4.20 & 0.15 & -4.14 & 0.12 \\
\hline cyclohexane & -2.31 & -2.80 & 0.09 & -3.66 & 0.13 & -3.66 & 0.15 \\
\hline cyclopentane & -1.80 & -2.33 & 0.08 & -2.78 & 0.09 & -2.83 & 0.11 \\
\hline decane & -3.48 & -3.88 & 0.14 & -5.28 & 0.25 & -5.40 & 0.31 \\
\hline ethanol & -4.36 & -2.85 & 0.06 & -3.27 & 0.10 & -3.28 & 0.11 \\
\hline ethylbenzene & -4.67 & -4.64 & 0.12 & -5.46 & 0.11 & -5.50 & 0.14 \\
\hline heptane & -2.22 & -2.57 & 0.11 & -3.14 & 0.11 & -3.18 & 0.14 \\
\hline hexane & -1.63 & -1.97 & 0.11 & -2.56 & 0.11 & -2.70 & 0.13 \\
\hline methanol & -4.13 & -2.14 & 0.06 & -2.38 & 0.08 & -2.40 & 0.08 \\
\hline methyl_tert-butyl_ether & -2.69 & -2.94 & 0.11 & -3.67 & 0.15 & -3.72 & 0.15 \\
\hline m-xylene & -4.80 & -4.88 & 0.09 & -5.67 & 0.10 & -5.90 & 0.13 \\
\hline nonane & -3.09 & -3.66 & 0.12 & -4.94 & 0.20 & -4.98 & 0.19 \\
\hline n-pentane & -1.00 & -1.38 & 0.10 & -1.95 & 0.12 & -1.97 & 0.11 \\
\hline octane & -2.66 & -3.02 & 0.11 & -3.88 & 0.11 & -3.99 & 0.14 \\
\hline o-xylene & -4.92 & -4.79 & 0.10 & -5.64 & 0.10 & -5.79 & 0.18 \\
\hline p-xylene & -4.78 & -4.81 & 0.09 & -5.82 & 0.15 & -5.84 & 0.13 \\
\hline tetrahydrofuran & -3.68 & -3.59 & 0.07 & -4.12 & 0.09 & -4.09 & 0.11 \\
\hline thiophene & -4.14 & -3.95 & 0.10 & -4.48 & 0.12 & -4.56 & 0.14 \\
\hline toluene & -4.33 & -4.01 & 0.13 & -4.62 & 0.13 & -4.78 & 0.15 \\
\hline RMSE & & 0.78 & & 1.18 & & 1.24 & \\
\hline MSE & & 0.02 & & 0.74 & & 0.81 & \\
\hline MAE & & 0.57 & & 1.07 & & 1.13 & \\
\hline$\tau$ & & 0.56 & & 0.53 & & 0.53 & \\
\hline PI & & 0.74 & & 0.67 & & 0.67 & \\
\hline
\end{tabular}


Table S10. Solvation free energies in [EMIM][OTf] with the charge scaling factor of $0.7 \mathrm{in} \mathrm{kcal} / \mathrm{mol}$. $\Delta G_{W_{a}}$ is the slow-growth estimate, $\Delta G_{\mathrm{EXP}}$ represents exponential averaging, and $\Delta G_{\mathrm{GEXP}}$ is its Gaussian approximation. SD represents the standard error of the free energy estimate, which is obtained from numerical bootstrap resampling. MSE, MAE, RMSE, $\tau$, and PI serve as quality measurements.

\begin{tabular}{|c|c|c|c|c|c|c|c|}
\hline Solute & Experiment & $\Delta G_{W_{a}}$ & $\mathrm{SD}$ & $\Delta G_{\mathrm{EXP}}$ & $\mathrm{SD}$ & $\Delta G_{\mathrm{GEXP}}$ & SD \\
\hline 1-butanol & -5.41 & -3.80 & 0.15 & -5.21 & 0.22 & -5.37 & 0.25 \\
\hline 1-heptyne & -3.60 & -3.93 & 0.18 & -5.19 & 0.21 & -5.52 & 0.19 \\
\hline 1-hexene & -1.92 & -0.87 & 0.16 & -1.84 & 0.11 & -2.08 & 0.15 \\
\hline 1-hexyne & -3.16 & -3.39 & 0.12 & -4.56 & 0.22 & -4.68 & 0.20 \\
\hline 1-octene & -2.73 & -1.50 & 0.17 & -3.46 & 0.34 & -3.66 & 0.35 \\
\hline 1-octyne & -4.06 & -3.86 & 0.14 & -5.92 & 0.24 & -6.10 & 0.37 \\
\hline 1-pentene & -1.45 & -0.27 & 0.09 & -1.14 & 0.10 & -1.28 & 0.12 \\
\hline 1-pentyne & -2.64 & -3.04 & 0.08 & -4.02 & 0.13 & -4.03 & 0.17 \\
\hline 1-propanol & -4.82 & -3.16 & 0.11 & -3.90 & 0.08 & -4.10 & 0.14 \\
\hline benzene & -3.87 & -2.82 & 0.09 & -3.70 & 0.12 & -3.72 & 0.15 \\
\hline cyclohexane & -2.31 & -1.43 & 0.13 & -2.76 & 0.19 & -2.82 & 0.26 \\
\hline cyclopentane & -1.80 & -1.18 & 0.12 & -2.21 & 0.12 & -2.37 & 0.13 \\
\hline decane & -3.48 & -2.57 & 0.10 & -4.25 & 0.17 & -4.18 & 0.22 \\
\hline ethanol & -4.36 & -2.96 & 0.08 & -3.71 & 0.13 & -3.71 & 0.12 \\
\hline ethylbenzene & -4.67 & -3.26 & 0.11 & -4.56 & 0.10 & -4.88 & 0.20 \\
\hline heptane & -2.22 & -1.02 & 0.10 & -2.57 & 0.20 & -2.67 & 0.29 \\
\hline hexane & -1.63 & -0.92 & 0.14 & -2.12 & 0.19 & -2.33 & 0.19 \\
\hline methanol & -4.13 & -2.31 & 0.06 & -2.73 & 0.05 & -2.79 & 0.06 \\
\hline methyl_tert-butyl_ether & -2.69 & -1.24 & 0.14 & -2.53 & 0.24 & -2.69 & 0.26 \\
\hline m-xylene & -4.80 & -4.38 & 0.12 & -5.33 & 0.16 & -5.38 & 0.20 \\
\hline nonane & -3.09 & -1.95 & 0.16 & -3.89 & 0.40 & -3.95 & 0.34 \\
\hline n-pentane & -1.00 & -0.29 & 0.09 & -1.52 & 0.17 & -1.36 & 0.19 \\
\hline octane & -2.66 & -2.23 & 0.10 & -3.07 & 0.09 & -3.34 & 0.14 \\
\hline o-xylene & -4.92 & -3.60 & 0.20 & -5.65 & 0.34 & -5.77 & 0.38 \\
\hline p-xylene & -4.78 & -3.45 & 0.13 & -4.70 & 0.09 & -4.90 & 0.16 \\
\hline tetrahydrofuran & -3.68 & -3.19 & 0.09 & -4.13 & 0.17 & -4.20 & 0.17 \\
\hline thiophene & -4.14 & -3.29 & 0.09 & -4.13 & 0.14 & -4.14 & 0.11 \\
\hline toluene & -4.33 & -3.15 & 0.11 & -4.19 & 0.20 & -4.33 & 0.19 \\
\hline RMSE & & 1.07 & & 0.79 & & 0.85 & \\
\hline MSE & & -0.90 & & 0.31 & & 0.43 & \\
\hline MAE & & 0.97 & & 0.61 & & 0.65 & \\
\hline$\tau$ & & 0.67 & & 0.63 & & 0.64 & \\
\hline PI & & 0.87 & & 0.81 & & 0.81 & \\
\hline
\end{tabular}


Table S11. Solvation free energies in [EMIM][OTf] with the charge scaling factor of $0.8 \mathrm{in} \mathrm{kcal} / \mathrm{mol}$. $\Delta G_{W_{a}}$ is the slow-growth estimate, $\Delta G_{\mathrm{EXP}}$ represents exponential averaging, and $\Delta G_{\mathrm{GEXP}}$ is its Gaussian approximation. SD represents the standard error of the free energy estimate, which is obtained from numerical bootstrap resampling. MSE, MAE, RMSE, $\tau$, and PI serve as quality measurements.

\begin{tabular}{|c|c|c|c|c|c|c|c|}
\hline Solute & Experiment & $\Delta G_{W_{a}}$ & $\mathrm{SD}$ & $\Delta G_{\mathrm{EXP}}$ & $\mathrm{SD}$ & $\Delta G_{\mathrm{GEXP}}$ & $\mathrm{SD}$ \\
\hline 1-butanol & -5.41 & -3.53 & 0.10 & -4.55 & 0.18 & -4.62 & 0.19 \\
\hline 1-heptyne & -3.60 & -2.70 & 0.18 & -4.66 & 0.37 & -5.07 & 0.32 \\
\hline 1-hexene & -1.92 & -0.17 & 0.10 & -1.17 & 0.17 & -1.23 & 0.18 \\
\hline 1-hexyne & -3.16 & -1.56 & 0.12 & -3.40 & 0.20 & -3.91 & 0.33 \\
\hline 1-octene & -2.73 & -0.48 & 0.17 & -2.24 & 0.43 & -2.42 & 0.32 \\
\hline 1-octyne & -4.06 & -2.79 & 0.16 & -4.69 & 0.34 & -4.96 & 0.43 \\
\hline 1-pentene & -1.45 & 1.39 & 0.15 & -0.13 & 0.22 & -0.58 & 0.42 \\
\hline 1-pentyne & -2.64 & -1.72 & 0.17 & -3.54 & 0.30 & -4.04 & 0.27 \\
\hline 1-propanol & -4.82 & -2.39 & 0.16 & -3.65 & 0.21 & -3.88 & 0.24 \\
\hline benzene & -3.87 & -2.10 & 0.10 & -3.29 & 0.20 & -3.42 & 0.18 \\
\hline cyclohexane & -2.31 & -0.45 & 0.14 & -1.97 & 0.15 & -2.26 & 0.19 \\
\hline cyclopentane & -1.80 & 0.48 & 0.18 & -1.17 & 0.26 & -1.47 & 0.32 \\
\hline decane & -3.48 & -0.20 & 0.18 & -2.50 & 0.27 & -3.21 & 0.42 \\
\hline ethanol & -4.36 & -2.48 & 0.09 & -3.29 & 0.10 & -3.41 & 0.15 \\
\hline ethylbenzene & -4.67 & -2.20 & 0.13 & -3.79 & 0.23 & -3.98 & 0.26 \\
\hline heptane & -2.22 & 0.16 & 0.14 & -1.60 & 0.16 & -2.37 & 0.29 \\
\hline hexane & -1.63 & 0.50 & 0.16 & -0.78 & 0.13 & -1.18 & 0.21 \\
\hline methanol & -4.13 & -2.63 & 0.13 & -3.43 & 0.16 & -3.42 & 0.14 \\
\hline methyl_tert-butyl_ether & -2.69 & -0.69 & 0.14 & -2.14 & 0.10 & -2.64 & 0.22 \\
\hline m-xylene & -4.80 & -2.57 & 0.16 & -4.20 & 0.13 & -4.96 & 0.25 \\
\hline nonane & -3.09 & -0.55 & 0.17 & -2.63 & 0.38 & -2.85 & 0.36 \\
\hline n-pentane & -1.00 & 0.45 & 0.11 & -0.90 & 0.17 & -1.01 & 0.23 \\
\hline octane & -2.66 & 0.23 & 0.12 & -1.43 & 0.13 & -2.24 & 0.39 \\
\hline o-xylene & -4.92 & -2.56 & 0.16 & -4.49 & 0.40 & -4.66 & 0.32 \\
\hline p-xylene & -4.78 & -2.73 & 0.17 & -4.47 & 0.22 & -5.05 & 0.20 \\
\hline tetrahydrofuran & -3.68 & -1.67 & 0.13 & -3.30 & 0.27 & -3.52 & 0.25 \\
\hline thiophene & -4.14 & -2.37 & 0.17 & -4.48 & 0.41 & -4.37 & 0.39 \\
\hline toluene & -4.33 & -2.40 & 0.17 & -4.02 & 0.23 & -4.42 & 0.35 \\
\hline RMSE & & 2.09 & & 0.74 & & 0.64 & \\
\hline MSE & & -2.02 & & -0.44 & & -0.11 & \\
\hline MAE & & 2.02 & & 0.67 & & 0.50 & \\
\hline$\tau$ & & 0.70 & & 0.69 & & 0.65 & \\
\hline PI & & 0.92 & & 0.89 & & 0.87 & \\
\hline
\end{tabular}

